\title{
Propagation of waves in porous media
}

\author{
M. YAVUZ CORAPCIOGLU and KAGAN TUNCAY
}

\begin{abstract}
Wave propagation in porous media is of interest in various diversified areas of science and engineering. The theory of the phenomenon has been studied extensively in soil mechanics, seismology, acoustics, earthquake engineering, ocean engineering, geophysics, and many other disciplines. This review presents a general survey of the literature within the context of porous media mechanics. Following a review of the Biot's theory of wave propagation in linear, elastic, fluid saturated porous media which has been the basis of many analyses, we present various analytical and numerical solutions obtained by several researchers. Biot found that there are two dilatational waves and one rotational wave in a saturated porous medium. It has been noted that the second kind of dilatational wave is highly attenuated and is associated with a diffusion type process. The influence of coupling between two phases has a decreasing effect on the first kind wave and an increasing effect on the second wave. Procedures to predict the liquefaction of soils due to earthquakes have been reviewed in detail. Extension of Biot's theory to unsaturated soils has been discussed, and it was noted that, in general, equations developed for saturated media were employed for unsaturated media by replacing the density and compressibility terms with modified values for a water-air mixture. Various approaches to determine the permeability of porous media from attenuation of dilatational waves have been described in detail. Since the prediction of acoustic wave speeds and attenuations in marine sediments has been extensively studied in geophysics, these studies have been reviewed along with the studies on dissipation of water waves at ocean bottoms. The mixture theory which has been employed by various researchers in continuum mechanics is also discussed within the context of this review. Then, we present an alternative approach to obtain governing equations of wave propagation in porous media from macroscopic balance equations. Finally, we present an analysis of wave propagation in fractured porous media saturated by two immiscible fluids.
\end{abstract}

\section{Introduction}

The dynamic response of porous media is of interest in various areas of engineering and physics. Underground nuclear explosions generate shock waves propagating through the porous medium surrounding the blast. Liquefaction of saturated sands due to dynamic loads has been studied extensively in earthquake engineer- 
ing. Attenuation of waves in geologic formations is of importance in seismic studies at very low frequencies $(1-100 \mathrm{~Hz})$. On the other hand, full-wave acoustic logging requires a much higher frequency range, almost up to $100 \mathrm{KHz}$. Elastic wave propagation in wet paper layers or the articulating cartilage is modeled as dynamic loads moving with a velocity across a poroelastic layer. Propagation of tidal fluctuations through groundwater aquifers or wave induced pressure fluctuations at ocean bottoms are other examples of wave propagation in porous media. Although liquid saturated materials attenuate waves gradually, dry porous materials exhibit pore crushing and pore collapse. Shock-wave compaction of porous metals has received considerable attention in mechanical engineering. Furthermore, due to their energy absorption characteristics, dry porous substances such as elastomeric foam are used as shock attenuators for commercial packaging purposes. Finally, one can note the soil-structure interaction due to pile driving, rotary machines, and moving heavy traffic in geotechnical engineering as examples of wave propagation in porous materials.

As seen in this brief listing of areas where the response of porous materials to dynamic loads plays a role, the study of wave propagation in porous media would cover a large number of disciplines. However, the conservation of mass and momentum principles form the basis of an analysis of problems arising in many diversified fields. In this theory, the deformable porous medium is viewed as a continuum consisting of a solid phase (either compressible or incompressible) and one or more fluids (gases and liquids). The solid phase constitutes the solid matrix with interconnected void space filled by fluids. These relations are introduced into the conservation of linear momentum and solid and fluid mass balance equations. Elastic constants of the constitutive relations are either obtained experimentally (e.g., Biot and Willis, 1957) or determined theoretically employing a theory such as that used by Duffy and Mindlin (1957). In this study, the governing equations of the phenomenon will be presented at a macroscopic level. That is, they are obtained by averaging the microscopic equations which are valid at a point within an individual phase present in the system over a representative elementary volume (REV) of the porous medium.

An alternative to continuum approach is the "distinct element" approach which treats the granular medium as an assemblage of individual particles with a particular geometry (circular disks, spheres, etc.). This approach employs Newtonian rigid body mechanics to simulate the translational and rotational motion of each element under dynamic loading. Dynamic photoelastic experiments provide experimental information (Sadd et al., 1989; Shukla and Zhu, 1988). This type of modeling effort started with Iida (1939) and included researchers like Gassman (1951), Brandt (1955), Duffy and Mindlin (1957), Goodman and Cowin (1972), Nunziato et al. (1978), Schwartz (1984), Sadd and Hossain (1989), and Chang et al. (1992) among many others. Since it is beyond the scope of this research, distinct element approach will not be covered in this review. The reader is referred to any one of these references for a more detailed treatment of the subject.

Therefore, it is the purpose of this review to present various continuum approach methodologies to formulate the wave propagation in porous media in different fields of interest. We will try to achieve this in such a way that the reader 
unfamiliar with the subject can follow the material within the context of porous media mechanics.

\section{Biot's theory}

Although the wave propagation in porous media has been studied quite some time, Biot's (1956a, b) work on wave propagation appears to be the first one employing the fundamentals of porous media mechanics. In addition to his dynamic theory, Biot (1941) also presented a quasi-static theory for elastic porous solids saturated with a fluid. Biot's work dominated the field over three decades, and influenced the direction of future research more than any other person who ever worked in this area. Not only the work published in the Western literature, but also research conducted by Russian researchers was affected by Biot's theory. For a review of Russian literature, we refer the reader to Nikolaevskij (1990). To do justice to this most significant work, we will start our review by presenting the Biot's formulation.

\subsection{Stress-strain relationships for a fluid saturated elastic porous medium}

The deformation of a porous medium can be related to the average displacement fields by using the theory of infinitesimal strain, i.e., the second and higher order displacement derivatives are neglected. Introducing the fundamental notation, the components of strain tensor of the solid matrix are

$$
\begin{aligned}
& \epsilon_{x x}=\frac{\partial u_{x}}{\partial x}, \quad \epsilon_{y y}=\frac{\partial u_{\mathrm{y}}}{\partial y}, \quad \epsilon_{z z}=\frac{\partial u_{z}}{\partial z} \\
& \gamma_{x y}=\frac{\partial u_{x}}{\partial y}+\frac{\partial u_{y}}{\partial x}, \quad \gamma_{x z}=\frac{\partial u_{z}}{\partial x}+\frac{\partial u_{x}}{\partial z}, \quad \gamma_{y z}=\frac{\partial u_{y}}{\partial z}+\frac{\partial u_{z}}{\partial y}
\end{aligned}
$$

The dilatation, $\boldsymbol{\epsilon}$, is expressed in terms of displacement vector, $\boldsymbol{u}$, as

$$
\epsilon=\nabla \cdot u
$$

The components of the rotation vector, $\omega$, are expressed as

$$
\omega_{\mathrm{x}}=\frac{1}{2}\left(\frac{\partial u_{z}}{\partial y}-\frac{\partial u_{y}}{\partial z}\right), \quad \omega_{y}=\frac{1}{2}\left(\frac{\partial u_{x}}{\partial z}-\frac{\partial u_{z}}{\partial x}\right), \quad \omega_{z}=\frac{1}{2}\left(\frac{\partial u_{y}}{\partial x}-\frac{\partial u_{x}}{\partial y}\right)
$$

and the rotation $\omega$ of the solid is given by

$$
\omega=\frac{1}{2} \nabla x u
$$

if $\omega=0$, the strain is irrotational. Similarly, the components $U_{x}, U_{y}$, and $U_{z}$ of the fluid displacement vector $\boldsymbol{U}$ are related to the dilatation of the fluid 


$$
e=\nabla \cdot U
$$

Similarly, the rotation in the fluid is given by

$$
\boldsymbol{\Omega}=\frac{1}{2} \nabla x \boldsymbol{U}
$$

It should be pointed out that this expression is not the actual strain in the fluid but simply the divergence of the fluid displacement which itself is derived from the average volume flow through the pores.

Following Biot (1956a), we assume that the solid skeleton of the porous medium is isotropic and for the relatively small deviations it is perfectly elastic. For such a solid matrix, the stress-strain relationships are expressed by

$$
\begin{aligned}
\sigma_{x x} & =2 N \epsilon_{x x}+A \epsilon+Q e \\
\sigma_{y y} & =2 N \epsilon_{y y}+A \epsilon+Q e \\
\sigma_{z z} & =2 N \epsilon_{z z}+A \epsilon+Q e \\
\tau_{x y} & =N \gamma_{x y} \\
\tau_{x z} & =N \gamma_{x z} \\
\tau_{y z} & =N \gamma_{y z}
\end{aligned}
$$

where

$$
\boldsymbol{\epsilon}=\epsilon_{x x}+\epsilon_{y y}+\epsilon_{z z}
$$

where $\sigma_{x x}, \sigma_{y y}$, and $\sigma_{z z}$ are the normal stresses in $x, y$, and $x$, directions, respectively. $\tau_{x y}, \tau_{x z}$, and $\tau_{y z}$ are the shear stresses. $\epsilon$ and $e$ denote the dilatation in the solid matrix and the fluid, respectively. The coefficient $N$ represents the shear modulus of the solid. The coefficients $A$ and $N$ correspond to Lame constants. In the theory of elasticity, they are denoted by $\lambda$ and $G$, respectively. The coefficient $Q$ is the cross coupling term between the volume changes of the solid matrix and the fluid. The stress in the fluid, $\sigma$, is proportional to the fluid pressure $p$ by

$$
-\sigma=n p
$$

where $n$ is the effective porosity which represents the interconnected pore space. Biot considers the sealed pore space as part of the solid. Note that solid stresses are positive in tension, and the fluid pressure is positive in compression. Although Biot neglects shear stresses in the fluid due to viscosity. Then the stress-strain relationship for the fluid is given by

$$
\sigma=Q \epsilon+R e
$$

where $R$ is defined by Biot as a measure of the fluid pressure to force a certain volume of the fluid into the porous medium while the total volume of the porous medium stays constant. If a stress is applied to the solid matrix while the fluid pressure is zero, the dilatation of the solid (and an associated decrease in porosity) would produce a reverse dilatation (volume expansion) of the fluid. As seen in 
equation (2.16), the ratio of dilatation would be equal to the ratio of $Q / R$. Fatt (1959) determined the values of four constants, $A, N, Q$, and $R$, by experiments of kerosene flow through Boise sandstone. Biot and Willis (1957) express $A, N$, $Q$, and $R$ as

$$
\begin{aligned}
& Q=\frac{n(\alpha-n)}{\gamma+\alpha \alpha^{*}} \\
& R=\frac{n^{2}}{\gamma+\alpha \alpha^{*}} \\
& A=\frac{\gamma / K+n^{2}+\alpha(1-2 n)}{\gamma+\alpha \alpha^{2}}-\frac{2 G}{3} \\
& N=G
\end{aligned}
$$

where $\alpha=1-\alpha^{*} / K, \gamma=n\left(1 / \beta-\alpha^{*}\right), G$ is the shear modulus of the solid matrix, $\alpha^{*}$ is the unjacketed compressibility (grain compressibility) which is the inverse of bulk modulus of solid grains, $K$ is the jacketed compressibility. Geertsma (1957) and Stoll (1979) also evaluated Biot's coefficients. The frequency dependence of the elastic moduli was investigated by Schmidt (1988)

\subsection{Equations of motion}

Biot (1956a) derived his theory of wave propagation in porous media by introducing the Lagrangian viewpoint and the concept of generalized coordinates. In Lagrangian description, one follows the movement of the REV rather than interpreting in terms of what happens at a fixed REV (Eulerian description). In this case, kinetic energy function, $T$, and dissipation function, $D$, are expressed in terms of six displacement components of solid and fluid phases. The kinetic energy, $T$, of the saturated isotropic porous medium per unit volume is expressed as

$$
\begin{aligned}
2 T= & \rho_{11}\left[\left(\frac{\partial u_{x}}{\partial t}\right)^{2}+\left(\frac{\partial u_{y}}{\partial t}\right)^{2}+\left(\frac{\partial u_{z}}{\partial t}\right)^{2}\right]+2 \rho_{12}\left[\frac{\partial u_{x}}{\partial t} \frac{\partial U_{x}}{\partial t}+\frac{\partial u_{y}}{\partial t} \frac{\partial U_{y}}{\partial t}+\frac{\partial u_{z}}{\partial t} \frac{\partial U_{z}}{\partial t}\right] \\
& +\rho_{22}\left[\left(\frac{\partial U_{x}}{\partial t}\right)^{2}+\left(\frac{\partial U_{y}}{\partial t}\right)^{2}+\left(\frac{\partial U_{z}}{\partial t}\right)^{2}\right]
\end{aligned}
$$

$T$ depends only on six displacement components. The dissipation depends on the relative movement between the solid and the fluid. When there is no relative motion, the dissipation function, $D$, vanishes. Then

$$
2 D=b\left[\left(\frac{\partial u_{x}}{\partial t}-\frac{\partial U_{x}}{\partial t}\right)^{2}+\left(\frac{\partial u_{y}}{\partial t}-\frac{\partial U_{y}}{\partial t}\right)^{2}+\left(\frac{\partial u_{z}}{\partial t}-\frac{\partial U_{z}}{\partial t}\right)^{2}\right]
$$

where $b$ is a constant (drag coefficient) for an isotropic medium and it is related to the fluid viscosity $\mu_{\mathrm{f}}$ and permeability $k$ by 


$$
b=\frac{\mu_{\mathrm{f}} n^{2}}{k}
$$

Biot calls $\rho_{11}, \rho_{12}$, and $\rho_{22}$ "mass coefficients", and they are related to densities of solid $\left(\rho_{\mathrm{s}}\right)$ and fluid $\left(\rho_{\mathrm{f}}\right)$ phases by

$$
\begin{aligned}
& \rho_{11}+\rho_{12}=(1-n) \rho_{\mathrm{s}} \\
& \rho_{12}+\rho_{22}=n \rho_{\mathrm{f}}
\end{aligned}
$$

The coefficient $\rho_{12}$ represents the mass coupling parameter (virtual mass effect) between the fluid and the solid phases and is always negative.

Then, if we denote the total force acting on the solid and fluid phases per unit volume in the $x$-direction by $F_{x}^{\mathbf{s}}$ and $F_{x}^{\mathrm{f}}$, respectively, we can derive the following from Lagrange's equations

$$
\begin{aligned}
& F_{x}^{\mathrm{s}}=\frac{\partial}{\partial t}\left[\frac{\partial T}{\partial\left(\partial u_{x} / \partial t\right)}\right]+\frac{\partial D}{\partial\left(\partial u_{x} / \partial t\right)}=\frac{\partial^{2}}{\partial t^{2}}\left(\rho_{11} u_{x}+\rho_{12} U_{x}\right)+b \frac{\partial}{\partial t}\left(u_{x}-U_{x}\right) \\
& F_{x}^{\mathrm{f}}=\frac{\partial}{\partial t}\left[\frac{\partial T}{\partial\left(\partial U_{x} / \partial t\right)}\right]+\frac{\partial D}{\partial\left(\partial U_{x} / \partial t\right)}=\frac{\partial^{2}}{\partial t^{2}}\left(\rho_{12} u_{x}+\rho_{22} U_{x}\right)-b \frac{\partial}{\partial t}\left(u_{x}-U_{x}\right)
\end{aligned}
$$

Similar equations may be written in the $y$ - and $z$-directions. If we express the force components, $F_{x}^{\mathrm{s}}$ and $F_{x}^{\mathrm{f}}$ as stress gradients, equations (2.21) and (2.22) can be rewritten in the following form

$$
\begin{aligned}
& \frac{\partial \sigma_{x z}}{\partial x}+\frac{\partial \tau_{x y}}{\partial y}+\frac{\partial \tau_{x z}}{\partial z}=\frac{\partial^{2}}{\partial t^{2}}\left(\rho_{11} u_{x}+\rho_{12} U_{x}\right)+b \frac{\partial}{\partial t}\left(u_{x}-U_{x}\right) \\
& \frac{\partial \sigma}{\partial x}=\frac{\partial^{2}}{\partial t^{2}}\left(\rho_{12} u_{x}+\rho_{22} U_{x}\right)-b \frac{\partial}{\partial t}\left(u_{x}-U_{x}\right)
\end{aligned}
$$

Since stresses are related to displacements by employing equations (2.8)-(2.16), the equations of motion can be stated as

$$
\begin{aligned}
& N \nabla^{2} u_{x}+(A+N) \frac{\partial \epsilon}{\partial x}+Q \frac{\partial e}{\partial x}=\frac{\partial^{2}}{\partial t^{2}}\left(\rho_{11} u_{x}+\rho_{12} U_{x}\right)+b \frac{\partial}{\partial t}\left(u_{x}-U_{x}\right) \\
& N \nabla^{2} u_{y}+(A+N) \frac{\partial \epsilon}{\partial y}+Q \frac{\partial e}{\partial y}=\frac{\partial^{2}}{\partial t^{2}}\left(\rho_{11} u_{y}+\rho_{12} U_{y}\right)+b \frac{\partial}{\partial t}\left(u_{y}-U_{y}\right) \\
& N \nabla^{2} u_{z}+(A+N) \frac{\partial \epsilon}{\partial z}+Q \frac{\partial e}{\partial z}=\frac{\partial^{2}}{\partial t^{2}}\left(\rho_{11} u_{z}+\rho_{12} U_{z}\right)+b \frac{\partial}{\partial t}\left(u_{z}-U_{z}\right) \\
& Q \frac{\partial \epsilon}{\partial x}+R \frac{\partial e}{\partial x}=\frac{\partial^{2}}{\partial t^{2}}\left(\rho_{12} u_{x}+\rho_{22} U_{x}\right)-b \frac{\partial}{\partial t}\left(u_{x}-U_{x}\right)
\end{aligned}
$$


TABLE 1

Frequency range of wave propagation in porous media

\begin{tabular}{llll}
$\begin{array}{l}\text { Frequency range } \\
\text { of Poiseuille flow }\end{array}$ & $\begin{array}{l}\text { Critical } \\
<\text { frequency, } f_{\mathrm{t}}\end{array}$ & $\begin{array}{l}\text { Characteristic } \\
\text { < frequency, } f_{\mathrm{c}}\end{array}$ & $\begin{array}{l}\text { High } \\
<\text { frequency range }\end{array}$ \\
& $\begin{array}{c}\mid \\
\mu_{\mathrm{f}}\end{array}$ & \begin{tabular}{c}
$\mu_{\mathrm{f}}=\mu_{\mathrm{f}}\left(f / f_{\mathrm{c}}\right)$ \\
\hline
\end{tabular} \\
\hline
\end{tabular}

$$
\begin{aligned}
& Q \frac{\partial \epsilon}{\partial y}+R \frac{\partial e}{\partial y}=\frac{\partial^{2}}{\partial t^{2}}\left(\rho_{12} u_{x}+\rho_{22} U_{y}\right)-b \frac{\partial}{\partial t}\left(u_{y}-U_{y}\right) \\
& Q \frac{\partial \epsilon}{\partial z}+R \frac{\partial e}{\partial z}=\frac{\partial^{2}}{\partial t^{2}}\left(\rho_{12} u_{z}+\rho_{22} U_{z}\right)-b \frac{\partial}{\partial t}\left(u_{z}-U_{z}\right)
\end{aligned}
$$

Equations (2.25)-(2.30) with six dependent variables, $u_{\mathrm{x}}, U_{\mathrm{x}}, u_{\mathrm{y}}, U_{y}, u_{z}$, and $U_{z}$, formulate the wave propagation in a fluid saturated isotropic porous medium. As noted by Biot (1956a), an acceleration of the solid matrix without any motion of fluid causes a pressure gradient in the fluid due to the coupling coefficient, $\rho_{12}$.

Biot assumed that the fluid flow $(U-u)$ relative to the solid is of the Poiseuille type. The coefficient $b$, as given by equation (2.19), is for Poiseuille flow. This assumption restricts the solution domain to low frequency range. For wave motions in high frequency range, Poiseuille flow assumption does not hold. At higher frequency range, a boundary layer develops on solid phase surfaces. The friction forces developing in this layer increases with frequency. The flow field in this layer is different than the flow beyond the boundary layer. Thus "the friction force of the fluid on the solid becomes out of phase with relative rate of flow and exhibits a frequency dependence" (Biot, 1962a). Biot (1956a) limits the lower frequency range with a "critical frequency" value, $f_{\mathrm{t}}$, defined by

$$
f_{\mathrm{t}}=\frac{\mu_{\mathrm{f}} \pi}{4 \rho_{\mathrm{f}} d^{2}}
$$

where $d$ is the diameter of the pores and $\mu_{\mathrm{f}}$ is the dynamic viscosity. By equation (2.31), the relative size of wave length of elastic waves is limited to an order of the pore diameter. This assumption can be avoided if we assume that the fluid is an ideal one. Furthermore, above the characteristic frequency, $f_{\mathfrak{c}}$, which depends on the kinematic viscosity of the fluid and the size of the pores, the viscosity must be considered frequency dependent (Table 1).

Since the use of Poiseuille flow concept is a major assumption in Biot formulation, we will briefly look at the Poiseuille equation. In fluid mechanics, steady state laminar flow due to pressure drop along a tube is called Poiseuille flow. The velocity distribution for such a flow in a tube with radius $r_{0}$ can be written in cylindrical coordinates. Let us take $x$-axis as the axis of the tube. The only velocity component, $u$, will be in the $x$-direction and will be independent of $x$. Then by neglecting gravity effects 


$$
-\frac{\partial p}{\partial x}+\mu_{\mathrm{f}} \frac{1}{r} \frac{\partial}{\partial r}\left(r \frac{\partial u}{\partial r_{2}}\right)=0
$$

By integrating equation (2.32) twice, employing boundary conditions at $r=0, u=$ $u_{\max }$ and at $r=r_{0}, u=0$, and noting that $u_{\text {ave }}=u_{\max } / 2$ where $u_{\text {ave }}$ is the average velocity, we obtain

$$
\frac{d p}{d x}=\frac{32 \mu_{\mathrm{f}}}{d^{2}} u_{\mathrm{ave}}=\frac{C \mu_{\mathrm{f}} u_{\mathrm{max}}}{R_{\mathrm{H}}^{2}}
$$

where $d$ is the diameter of the tube. Note that in equation (2.32) there is no inertia term. In equation $(2.33), R_{\mathbf{H}}$ is the hydraulic radius $(=d / 4$ for circular pipes), and $C$ is a constant (Kozeny's constant, or a shape factor). In porous media flow, $R_{\mathrm{H}}$ will be a measure of the size of pores. A comparison of equation (2.33) with equation (2.19) shows that for a porous medium $k=n R_{\mathrm{H}}^{2} / C$. Tiller (1975) illustrates the variation of Kozeny's constant with porosity. For $0<C<10$, $C$ can be calculated by

$$
C=\frac{3.5 n^{3}}{\sqrt{(1-n)}}\left[1+57(1-n)^{3}\right]
$$

\subsection{Derivation of dilatational wave propagation equations}

If we differentiate both sides of equation (2.25) with respect to $x$, both sides of equation (2.26) with respect to $y$, and both sides of equation (2.27) with respect to $z$ and add using equation (2.14) and assuming constant $\rho_{11}, \rho_{22}$, and $\rho_{12}$, we obtain

$$
\nabla^{2}(P \epsilon+Q e)=\frac{\partial^{2}}{\partial t^{2}}\left(\rho_{11} \epsilon+\rho_{12} e\right)+b \frac{\partial}{\partial t}(\epsilon-e)
$$

where $P=A+2 N$. If we perform same operations and make the same assumptions for equations $(2.28)-(2.30)$, then

$$
\nabla^{2}(Q \epsilon+R e)=\frac{\partial^{2}}{\partial t^{2}}\left(\rho_{12} \epsilon+\rho_{22} e\right)-b \frac{\partial}{\partial t}(\epsilon-e)
$$

Equations (2.34) and (2.35) govern the propagation of dilatational waves in a porous medium. These two equations clearly show the coupling between them.

If we neglect the dissipative forces, and consider purely elastic waves, i.e., $b=$ 0 in equations (2.34) and (2.35), the velocities of two dilatational waves, $V_{1}$ and $V_{2}$ are obtained from the solution of a quadratic equation by assuming solutions to equations (2.34) and (2.35) to be represented by

$$
\begin{aligned}
& \epsilon=C_{1} e^{i(\beta x+\theta t)} \\
& e=C_{2} e^{i(\beta x+\theta t)}
\end{aligned}
$$

The velocity, $V$, of these waves is $V=\beta / \theta$ which is determined by substituting $\epsilon$ and $e$ expressions into equations (2.34) and (2.35) as 


$$
\begin{aligned}
& \frac{P R-Q^{2}}{H^{2}} Z^{2}-\left[\frac{P R}{H^{2}}+\frac{R \rho_{11}-2 Q \rho_{12}}{H\left(\rho_{11}+\rho_{22}+2 \rho_{12}\right)}\right] Z+\left[\frac{\rho_{11} \rho_{22}-\rho_{12}^{2}}{\left(\rho_{11}+\rho_{22}+2 \rho_{12}\right)^{2}}\right]=0 \\
& V_{1}^{2}=\frac{H}{\left(\rho_{11}+\rho_{22}+2 \rho_{12}\right) Z_{1}}, \quad V_{2}^{2}=\frac{H}{\left(\rho_{11}+\rho_{22}+2 \rho_{12}\right) Z_{2}}
\end{aligned}
$$

where $H=P+R+2 Q \cdot \beta$ and $\theta$ denote to wave number and circular frequency, respectively. Biot designates the high-velocity compression wave as the wave of the "first kind", and the low-velocity wave as the wave of the "second kind". However, we must note that in general, neither of these waves propagate as a wave in the fluid or in the solid matrix alone, both travel jointly in the matrix and the fluid. If coupling is weak (see the solution of Garg et al. (1974) in section 3.1 ) waves propagate in a form which closely resemble a wave in the solid matrix alone, and a wave in the fluid alone.

Biot (1956a) demonstrated the possible existence of an elastic wave, in which no relative motion between the fluid and solid phases occurs $(\epsilon=e)$ and the dissipation due to fluid friction disappears. This is obtained when a "dynamic compatibility" condition is satisfied between the elastic and dynamic constants, i.e.

$$
\frac{(A+2 N+Q)\left(\rho_{11}+2 \rho_{12}+\rho_{2}\right)}{\left(\rho_{11}+\rho_{12}\right)(A+2 N+R+2 Q)}=\frac{(R+Q)\left(\rho_{11}+2 \rho_{12}+\rho_{22}\right)}{\left(\rho_{22}+\rho_{12}\right)(a+2 N+R+2 Q)}=1
$$

The propagation velocity of this wave is given by

$$
V^{2}=\frac{A+2 N+R+2 Q}{\rho_{11}+2 \rho_{12}+\rho_{22}}
$$

As $b$ increases (i.e., higher frequencies), $(\boldsymbol{u}-\boldsymbol{U})$ would decrease. This implies that both phases would eventually have the same velocity field. Then one might use a single velocity field and a single stress-strain relation. This would correspond to Biot's single elastic wave with no attenuation, satisfying the "compatibility condition".

If the dissipation is included, then the quadratic equation becomes

$$
\left(Z-Z_{1}\right)\left(Z-Z_{2}\right)+i M(Z-1)=0
$$

where $i=(-1)^{1 / 2}$, and $M$ is a frequency variable in terms of $b, P, R, Q, \rho_{11}, \rho_{22}$, $\rho_{12}$, and a characteristic frequency, $f_{\mathrm{c}}$

$$
f_{\mathrm{c}}=\frac{b}{2 \pi \rho_{\mathrm{f}} n}
$$

The characteristic frequency, $f_{\mathrm{c}}$ is proportional to the critical frequency, $f_{\mathrm{t}}$ [e.g., (2.31)] which defines the limit of Poiseuille flow. The proportionality depends on the detailed pore geometry. For pores represented by circular tubes,

$$
\frac{f_{\mathrm{t}}}{f_{\mathrm{c}}}=0.154
$$




\section{TABLE 2}

Coefficient combinations for Figures 1-6

\begin{tabular}{lllllllll}
\hline Case \# & $P / H$ & $R / H$ & $Q / H$ & $\rho_{11} / \rho$ & $\rho_{22} / \rho$ & $\rho_{12} / \rho$ & $Z_{1}$ & $Z_{2}$ \\
\hline 1 & 0.610 & 0.305 & 0.043 & 0.500 & 0.500 & 0 & 0.812 & 1.674 \\
2 & 0.610 & 0.305 & 0.043 & 0.666 & 0.333 & 0 & 0.984 & 1.203 \\
3 & 0.610 & 0.305 & 0.043 & 0.800 & 0.200 & 0 & 0.650 & 1.339 \\
4 & 0.610 & 0.305 & 0.043 & 0.650 & 0.650 & -0.150 & 0.909 & 2.394 \\
5 & 0.500 & 0.500 & 0 & 0.500 & 0.500 & 0 & 1.000 & 1.000 \\
6 & 0.740 & 0.185 & 0.037 & 0.500 & 0.500 & 0 & 0.672 & 2.736 \\
\hline
\end{tabular}

Where $H=P+R+2 Q, P=A+2 N, \rho=\rho_{11}+\rho_{22}+2 \rho_{12}$.

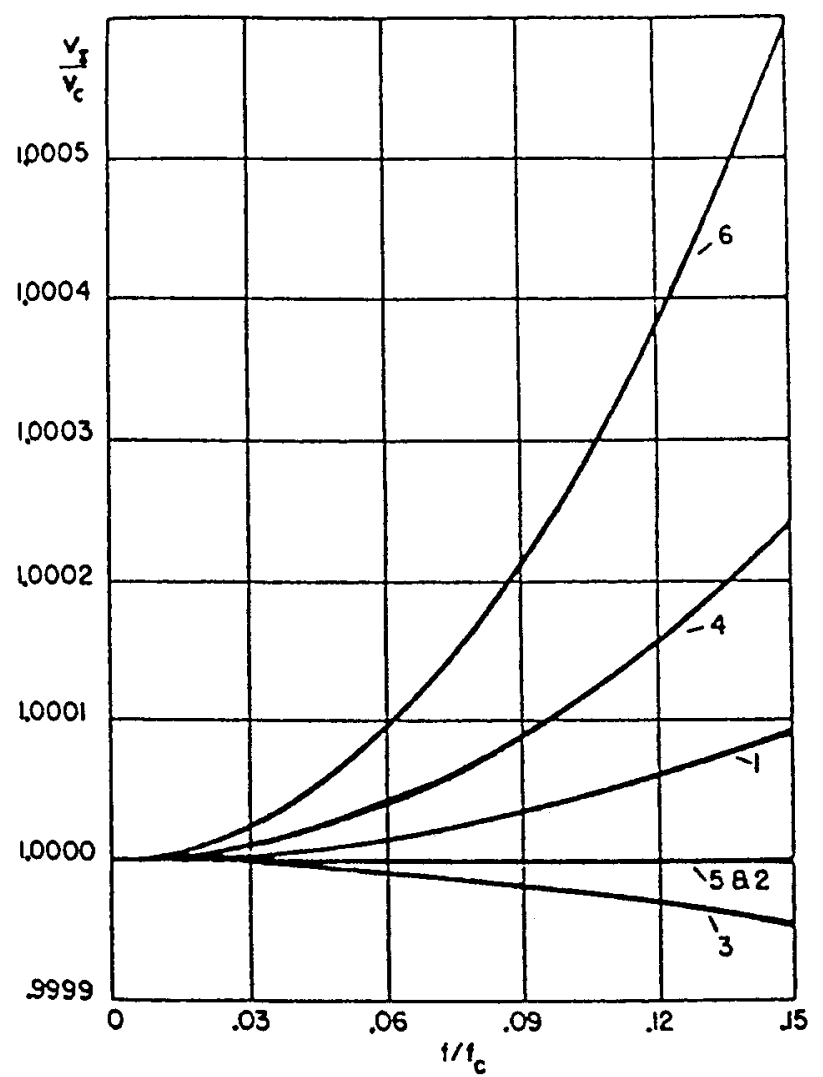

Fig. 1. Phase velocity $v_{1}$ of dilatational waves of the first kind (after Biot, 1956a).

At frequencies below characteristic frequency viscous forces, and above it inertial forces are significant with no coupling between the fluid and the solid (see Table 2).

The first root of equation (2.37) which reaches to unity at zero frequency corresponds to waves of the first kind while the second root corresponds to waves of the second kind. Biot (1956a) presents velocity expressions and their graphical representations in the range $0<f / f c<0.15$ (see Table 2 and Figs. 1-4). 


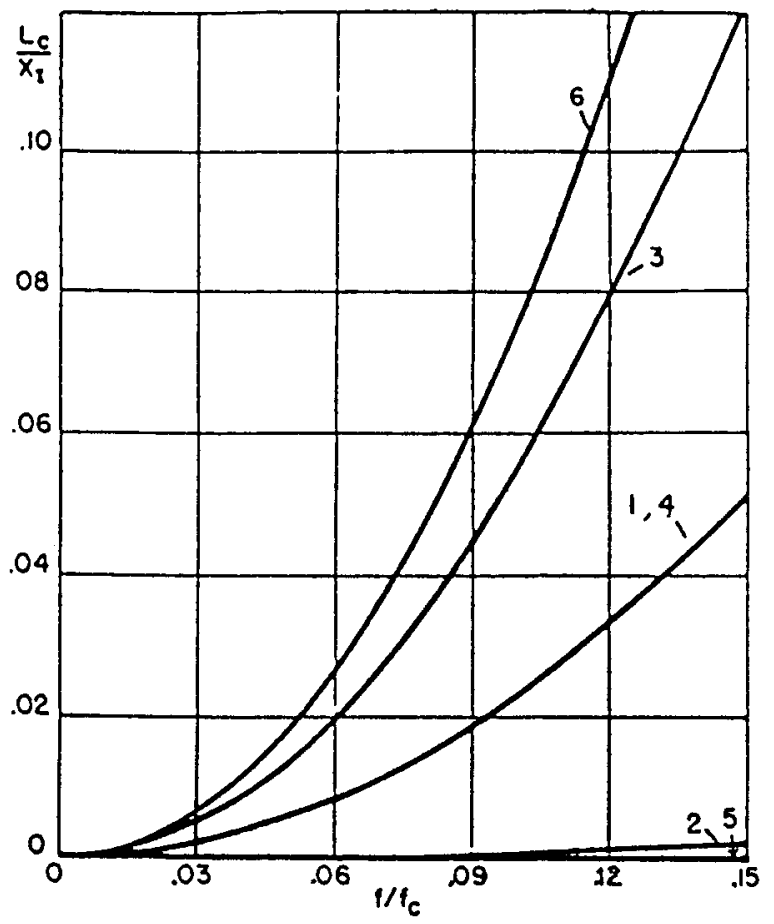

Fig. 2. Attenuation coefficient of dilatational waves of the first kind (after Biot, 1956a).

\subsection{Derivation of rotational wave propagation equations}

If we eliminate $[(A+N) \epsilon+Q e]$ between equations (2.25) and (2.26) by differentiating both sides of equation (2.25) with respect to $y$ and of equation (2.26) with respect to $x$ and subtract, we obtain an equation in terms of $\omega_{z}$ and $\Omega_{z}$. Similar equations are obtained for $\omega_{x}, \Omega_{x}, \omega_{y}$ and $\Omega_{y}$ from equations (2.26) and (2.27) and equations (2.25) and (2.27), respectively. By adding these three equations, we obtain the equation for rotational waves for the solid phase

$$
N \nabla^{2} \boldsymbol{\omega}=\frac{\partial^{2}}{\partial t^{2}}\left(\rho_{11} \omega+\rho_{12} \boldsymbol{\Omega}\right)+b \frac{\partial}{\partial t}(\boldsymbol{\omega}-\boldsymbol{\Omega})
$$

If we perform same operations for equations (2.28)-(2.30) we obtain the equation for rotational waves for the fluid phase

$$
\frac{\partial^{2}}{\partial t^{2}}\left(\rho_{12} \boldsymbol{\omega}+\rho_{22} \boldsymbol{\Omega}\right)-b \frac{\partial}{\partial t}(\boldsymbol{\omega}-\boldsymbol{\Omega})=0
$$

If we neglect the dissipation term in these equations, and eliminate $\boldsymbol{\Omega}$ from both, we obtain 


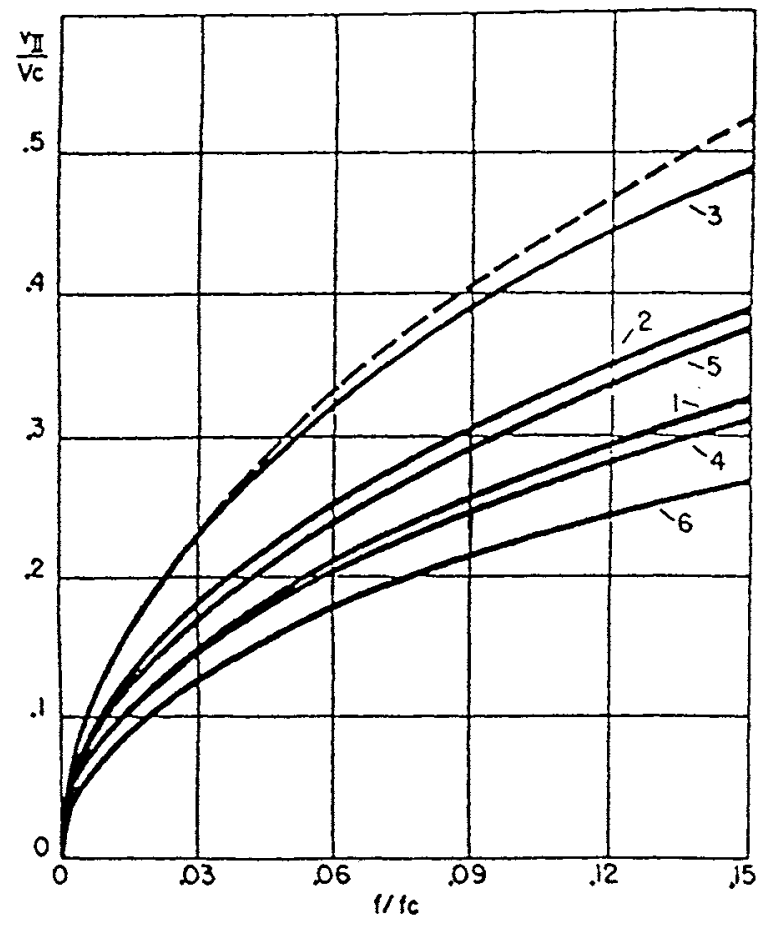

Fig. 3. Phase velocity $v_{\mathrm{II}}$ of dilatational waves of the second kind (after Biot, 1956a).

$$
N \nabla^{2} \omega=\rho_{11}\left[1-\frac{\rho_{12}^{2}}{\rho_{11} \rho_{12}}\right] \frac{\partial^{2} \omega}{\partial t^{2}}
$$

Equation (2.41) shows that there is only one type of rotational wave with velocity

$$
V_{\mathrm{c}}^{2}=\frac{N}{\rho_{11}\left[1-\frac{\rho_{12}^{2}}{\rho_{11} \rho_{22}}\right]}
$$

The rotation of the fluid, $\mathbf{\Omega}$ is calculated by

$$
\mathbf{\Omega}=-\frac{\rho_{12} \boldsymbol{\omega}}{\rho_{22}}
$$

Since $\rho_{12}<0, \boldsymbol{\Omega}$ and $\boldsymbol{\omega}$ are both in the same direction. Biot noted that although there is no circulation in a frictionless fluid at microscopic level, the line integral of the volume flow (volume of fluid per unit time per unit cross sectional area of the medium) can be nonzero. Since there is no coupling of rotational (shear) wave of the fluid and the solid phase, the shear waves in a saturated porous medium signifies the shear waves in the solid matrix. However, there is a strong effect of coupling involved in both compression waves (see Section 2.3), i.e., the presence of water exerts an influence on the dilatational wave velocities, but produces a very minor effect on the rotational wave velocity. 


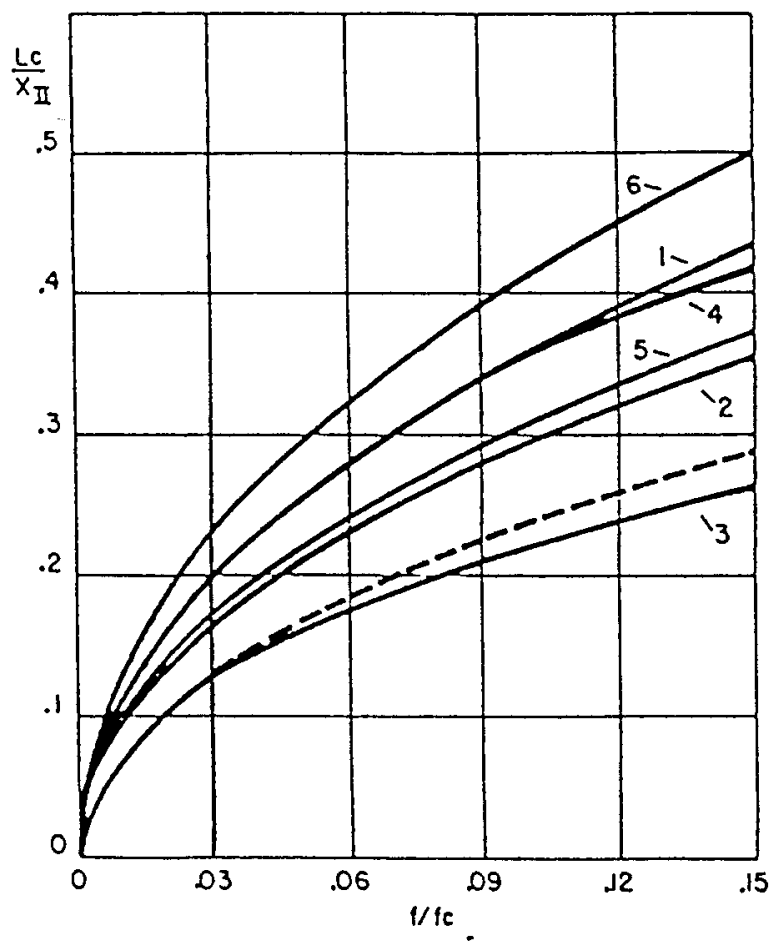

Fig. 4. Attenuation coefficient of dilatational waves of the second kind (after Biot, 1956a).

In seismology, compression waves known as primary or $P$ waves arrive first at some distance away from an earthquake. These waves are predominantly longitudinal, and followed by secondary (rotational) waves or $S$ waves corresponding to a transverse wave in which the direction of motion is normal to the direction of propagation. In an infinite isotropic saturated porous medium, there are only two types of waves $(P$ and $S$ ) that can be propagated. However, in regions close to surface, a third type of wave arrives after the body waves. These are known as Rayleigh waves and have both vertical and horizontal components. The former dominates, and the amplitude diminishes exponentially with depth. Love waves which appear due to stratification of the earth arrive last. Rayleigh waves have been studied by Jones (1961), Levy and Sanchez-Palencia (1977), Deresiewicz (1962), and Foda and Mei (1983) in homogeneous porous media, and by Beskos et al. (1989b) in fissured porous media. Love waves were investigated by Deresiewicz (1961, 1964a, 1965) and Chattopadhyay and De (1983). Variable frequency interface waves known as Stoneley waves have been analyzed by Stoll (1980) and Pascal (1986).

\subsection{Modifications of Biot's theory}

In a companion paper, Biot (1956b) extended his theory to high-frequency range. In this case the coefficient $b$ which is the ratio of the total friction force to the average relative fluid velocity for oscillatory motion is multiplied by frequency 
correction factor, $F(\kappa)$ which represents the deviation from Poiseuille friction as the frequency increases. He proposed various expressions for $F(\kappa)$ by assuming certain pore structures (e.g., parallel tubes in the direction of flow). In general

$$
F(\kappa)=F\left[\delta\left(\frac{f}{f_{\mathrm{c}}}\right)^{1 / 2}\right]
$$

where $\delta$ is the "structural factor" and $\mathrm{f}$ is the frequency of the wave and $f_{\mathrm{c}}$ is a characteristic frequency defined by equation (2.38). As noted by Biot (1962a) such a correction is also needed for mass coefficients, $\rho_{11}, \rho_{12}$, and $\rho_{22}$ to take into account the deviation from the Poiseuille flow. A functional form of $F(\kappa)$ is given by Stoll (1977) (see equation [7.10]).

Later, Biot (1962a) modified his original theory slightly by employing the general principles of nonequilibrium thermodynamics and extended to an anisotropic medium with an elastic matrix. Modifications include a new definition of $b$ as $\mu_{\mathrm{f}} / k$. The elastic coefficients $N, A, Q$, and $R$ were replaced by new elastic constants, $\left[\lambda+M(\alpha-n)^{2}\right], n(\alpha-n) M$, and $n^{2} M$ where $M=Q$. For an incompressible fluid $\alpha=1$. Equations (2.21) and (2.22) were replaced by

$$
\begin{aligned}
& \nabla \cdot \tau=\frac{d}{d t}\left[\frac{\partial T}{\partial(\partial u / \partial t)}\right] \\
& -\nabla p=\frac{d}{d t}\left[\frac{\partial T}{\partial(\partial w / \partial t)}\right]+\frac{\partial D}{\partial(\partial w / \partial t)}
\end{aligned}
$$

where

$$
w=n(U-u)
$$

For a uniform porosity, Biot called $\zeta=-\nabla . w$ "fluid content". Equation (2.46) takes into account the relative motion of the fluid with respect to the solid matrix. This approach is more in line with relative Darcy's law concept used in a deforming porous medium. It is the specific discharge relative to the moving solid that is expressed by Darcy's law. Biot started with a thermodynamic system initially under equilibrium to apply the thermodynamics of irreversible processes. The initial state was defined as the one with no pressure gradients or gravity forces acting on the fluid phase. A disequilibrium force was applied to perturb the system. Biot expressed this disequilibrium force in a form conjugate to the flow coordinate. Then, he used Onsager's principle to express Darcy's law in a three-dimensional space.

Biot's modified equations can be stated as follows with constant parameters.

$$
\begin{aligned}
& \mu \nabla^{2} u+(N+\lambda) \nabla \epsilon-\alpha M \nabla \zeta=\frac{\partial^{2}}{\partial t^{2}}\left(\rho u+\rho_{\mathrm{f}} w\right) \\
& \nabla(\alpha M \epsilon-M \zeta)=\frac{\partial^{2}}{\partial t^{2}}\left(\rho_{\mathrm{f}} u+m w\right)+\frac{\mu_{\mathrm{f}}}{k} \frac{\partial w}{\partial t}
\end{aligned}
$$

where $\rho=\rho_{11}+\rho_{22}+2 \rho_{12}=(1-n) \rho_{\mathrm{s}}+\rho_{\mathrm{f}} . m$ is mass coefficient for an isotropic 
medium and it is related to $\rho_{\mathrm{f}}$. If we multiply equation (2.49) by $n$ and subtract from equation (2.48), we obtain equations $(2.25-2.30)$.

Note that the term within the parentheses on the left had side of equation (2.49) is $p$. Also, $\rho_{11}=\rho-2 \rho_{\mathrm{f}} n+m n^{2}, \rho_{22}=m n^{2}$, and $\rho_{12}=\rho_{\mathrm{f}} n-m n^{2}$.

Equations of dilatational wave propagation can be given in terms of solid dilatation, $\epsilon$, and fluid content, $\zeta$ as

$$
\begin{aligned}
& \nabla^{2}(H \epsilon-\alpha M \zeta)=\frac{\partial^{2}}{\partial t^{2}}\left(\rho \epsilon-\rho_{\mathrm{f}} \zeta\right) \\
& \nabla^{2}(-\alpha M \epsilon+M \zeta)=\frac{\partial^{2}}{\partial t^{2}}\left(-\rho_{\mathrm{f}} \epsilon+m \zeta\right)+\frac{\mu_{\mathrm{f}}}{k} \frac{\partial \zeta}{\partial t}
\end{aligned}
$$

where $H=\lambda+2 N$. The coefficient $m$ is given by

$$
m=\delta \frac{\rho_{\mathrm{f}}}{n}
$$

where $\delta$ is the structural factor indicating the apparent increase in fluid inertia caused by the tortuosity of the pore space (Stoll and Bryan, 1970). For a random system of uniform pores, $\delta=3$. Berryman $(1980 \mathrm{a}, \mathrm{b})$ theoretically determined $\delta$ as

$$
\delta=1-r_{0}\left[1-\frac{1}{n}\right]
$$

where $r_{0}$ is a coefficient $\left(1>r_{0}>0\right) .\left(r_{0} \rho_{\mathrm{f}}\right)$ is the induced mass caused by the oscillation of solid particles in the fluid. Berryman noted that $r_{0}$ should be determined from microscopic models. Berryman's (1981a) switch from macroscopic displacement parameters used by Biot to microscopic fluid displacement (and strain) parameters caused him to arrive at erroneous results in his analysis (Korringa, 1981). Later Berryman (1981b) has shown that his error arose due to misinterpretation of fluid content, $\zeta$.

Hovem and Ingram (1979) used the real part of $F(\kappa)$ (see equation (7.10)) to multiply with $\mu_{\mathrm{f}}$ in equation (2.51). Also, they defined $m$ by

$$
m=\frac{\rho_{\mathrm{f}}}{n}\left[1+\frac{n \mu_{\mathrm{f}} F(\kappa)}{\rho_{\mathrm{f}} k f}\right]
$$

The term within the parentheses is the structural factor, $\delta$. Hovem and Ingram showed that for low frequencies

$$
\delta=1+\frac{R^{*}}{6}
$$

where $R^{*}$ is a coefficient taking into account the pore shape and the tortuosity of the pores (e.g., $R^{*}=2$ for circular tubes, $R^{*}=5$ for spherical grains). At high frequencies, coefficients $N, \lambda, \alpha, M, H, m$, and $\mu_{\mathrm{f}}$ are replaced by equivalent terms to introduce frequency dependance of these coefficients.

Biot (1962b) presented new features of the theory in more detail and generalized 
it by introducing a viscodynamic operator. In addition, a more detailed analysis of viscoelastic and solid dissipation is given.

\subsection{Elaboration on Biot's work by other researchers}

A large number of researchers modified, revised, and solved Biot's formulation. In this section we will review some of these works.

Hardin (1965) described column test studies for evaluating the damping in sands and gave an example of the application of Biot's theory to a water-saturated body of quartz sand. He also presented the application of Kelvin-Voight model (viscous damping) and found that this model satisfactorily represented the behavior of sands in small-amplitude vibration tests. Hardin and Richart (1963) showed that the shear modulus of soils is essentially dependent on various variables such as average effective confining pressure, void ratio, and frequency among others. They showed that the grain size and grading had almost no effect on shear modulus, and the degree of saturation had a minor effect only at low pressures. Allen et al. (1980) conducted laboratory experiments to determine Biot relationships between pore pressure, time, degree of saturation, and compression wave velocity. The effect of saturation has been investigated in detail by Santos et al. (1990a) (Section 5).

Ishihara (1967) revised Biot's theory and obtained dilatational wave propagation equations with dissipation in terms of $e$ and $\zeta$ [see equations (2.48) and (2.49)]. After eliminating $\zeta$ between two equations, they were reduced to a fourth-order differential equation in terms of wave velocity $V$. The material parameters of the resulting equation were expressed as functions of measurable quantities. By doing this, Ishihara redefined Biot coefficients in terms of basic compressibilities. This is similar to Geertsma and Smit's (1961) approach which redefined Biot's elastic constants in terms of compressibilities of the phases and porosity. Ishiara has shown that the wave of the first kind at low frequencies would travel without drainage of water, and its velocity can be calculated by using undrained tests. This also implies that there is no movement of water relative to the solid matrix and the first kind wave travel without causing pore volume change through the solidwater-system. At high frequencies (e.g., ultrasonic vibrations in soils) $f>f_{\mathrm{c}}$, the first kind waves cause rapid fluctuations in pore pressure due to strain, so that there is not enough time for water to drain due to pressure gradients and the attenuation disappears. The stress condition is a drained condition. Furthermore, at higher frequencies the wavelength is short, and therefore the travel distance for water is also short. However, at low frequencies, although there is enough time to travel, the distance is much larger due to larger wave length, thus, the drainage does not progress. The lack of drainage is not because of the movement of the wave as erroneously assumed by some engineers (Ishihara, 1967). Waves due to earthquakes and explosions are usually waves of the first kind at lowfrequencies.

The waves of the second kind usually correspond to consolidation deformation at low frequencies. In this kind of waves, wave energy is quickly lost due to large attenuations. Thus, the disturbance cannot travel as a wave but rather it propagates 


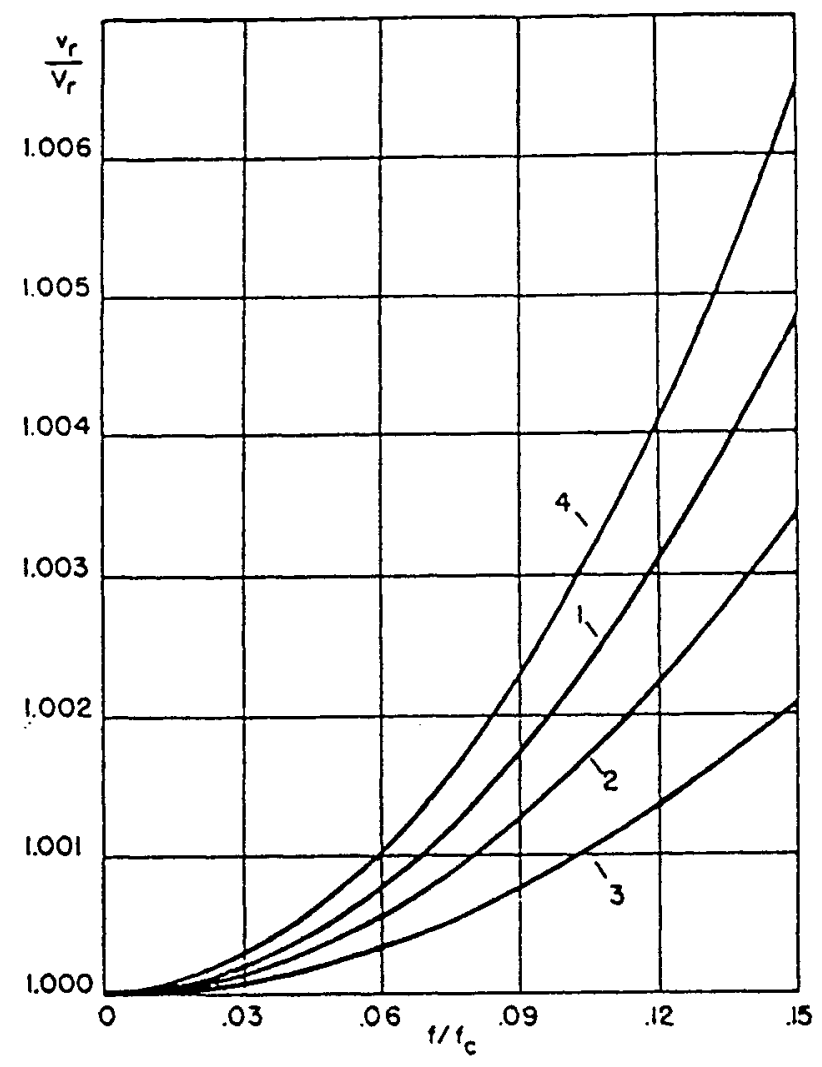

Fig. 5. Phase velocity $v_{\mathrm{r}}$ of rotational waves (after Biot, 1956a).

in a form similar to diffusion (i.e., consolidation) and the phase velocity is reduced to zero. The pore compressibility has predominant effect on the wave behavior. These waves can only progress where there is a change in pore volume. At higher frequencies, the disturbance travels as a wave under drained conditions similar to the first kind waves. Ishihara (1967) calculated the velocities of all four types of waves. The velocity of the wave of the first kind is the same as the one derived by Geerstma and Smit (1961).

A comparison of the first and second kind of waves and rotational waves can be illustrated in Figs. 1-6. The numbers in all figures refer to different combinations of Biot coefficients (see Table 2). In all cases $\rho_{12}=0$ except at case 4 which gives the highest rotational (shear) wave velocity due to cross coupling of fluid and solid phase rotations, $\boldsymbol{\omega}$ and $\boldsymbol{\Omega}$. Number 5 refers to $Q=0$, i.e., no cross coupling between the volume changes of the solid, $e$, and the fluid, $e$. Number 6 refers to a case with a large $(A+2 N)$ in comparison to other parameters, i.e., purely elastic dilatational waves, and no rotational waves. Case 6 also assumes equal fluid and solid masses $\rho_{11}=\rho_{22}$. Number 3 refers to a case with a large $\rho_{11}$, and $(A+2 N)$, i.e., low porosity medium. Since $\rho_{11}$ represents the total effective mass of solid moving in the fluid, case 3 waves mostly travel in the fluid. 


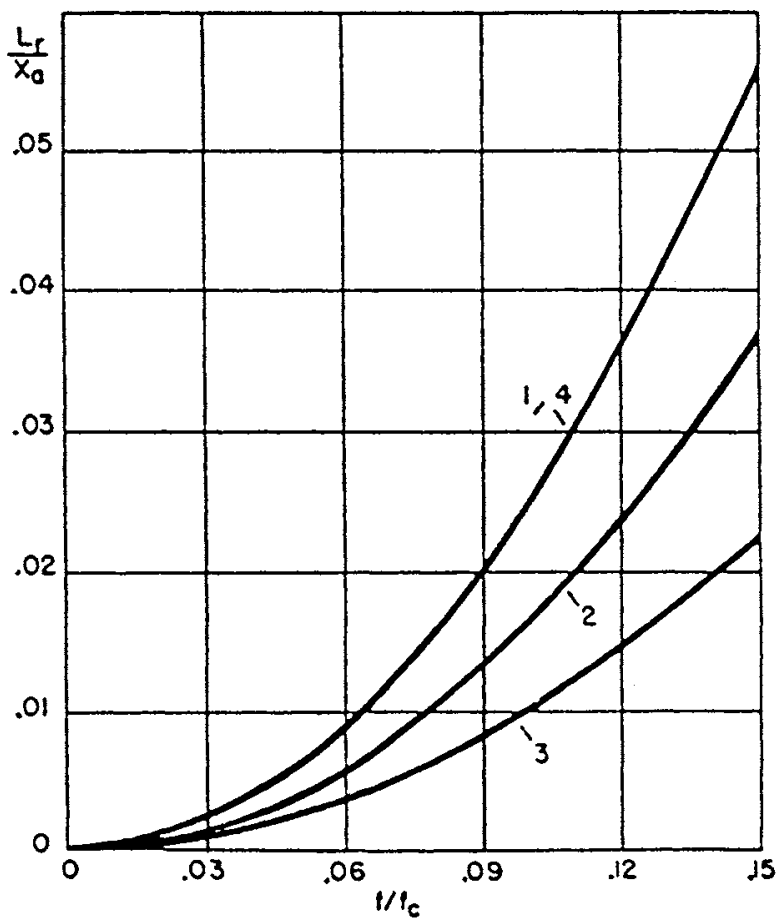

Fig. 6. Attenuation coefficient of rotational waves (after Biot, 1956a).

In summary, Biot found that the phase velocity of rotational waves increases slightly with frequency. The attenuation is proportional to the square of the frequency. The first kind dilatational waves are "true waves". The phase velocity changes with frequency depending on the elastic Biot coefficients. The attenuation is also proportional to the square of the frequency. When the dissipation due to fluid friction disappears, so does the attenuation of those waves. Dilatational waves of second kind attenuate highly. As noted earlier their propagation is diffusive and slower than that of the wave of the first kind.

In a series of ten papers, Deresiewicz and his coworkers (1960)-(1967) obtained solutions of various problems of wave reflection and refraction at the interfaces, and studied the effects of boundaries in a liquid saturated porous medium by using Biot's theory. Dunn (1986) investigated the effect of boundary conditions of a porous rock cylinder at low frequencies, and discovered the existence of an "artificial attenuation" caused by the open-pore boundary conditions. Lovera (1987) studied the boundary conditions for a fluid saturated porous solid. Wu et. al. (1990) and Santos et al. (1992) computed reflection and refraction coefficients for various interface conditions.

Sun et. al. (1993) studied harmonic wave propagation through an anisotropic, periodically layered porous medium by using Biot's theory to describe the constitutive relations. They presented results for a layered, fluid saturated, fabric material. Sharma and Gogna (1991b) employed Biot's theory to investigate the propagation of plane harmonic seismic waves in a transversely isotropic porous medium. They 
concluded that anisotropy has significant effect on the velocities of body waves. They also presented frequency equation for surface waves.

Albert (1993) compared propagation characteristic of water filled and air filled materials in $10 \mathrm{~Hz}-100 \mathrm{kHz}$ band. Analogous to an elastic medium, Deresiewicz (1962) and Jones (1961) independently showed the existence of surface waves in saturated porous media. They examined surface waves by considering the coupled transverse wave and one of the compressional waves. Later Tajuddin (1984) presented a study of Rayleigh waves considering all three types of body waves. Tajuddin (1984) extended the study to convex cylindrical pervious and impervious surfaces and found that phase velocity is higher for the impervious surface than for the pervious surface. Foda and Mei (1983) proposed a boundary layer theory for Rayleigh waves. Weng and Yew (1990) examined the behavior of leaky Rayleigh waves generated by a line source. Philippacopoulos (1987) investigated Rayleigh waves in partially saturated layered half spaces. However, we should note that his model consist of a saturated porous half space and a dry elastic layer. Hence, his results are not applicable to unsaturated porous media. Feng and Johnson (1983) numerically searched for the velocities of various surface modes at an interface between a fluid half space and a half space of a fluid saturated porous medium. Attenborough and Chen (1990) modified Biot's theory and obtained dispersion equations for a rigid porous half space, for a poroelastic half space, and for a layered poroelastic half space. They predicted the possibility of two additional types of surface waves at an air/air-filled poroelastic interface.

Love waves which appear due to stratification of the earth were studied by Deresiewicz (1961, 1964a, 1965) and Chattopadhyay and De (1983). Sharma and Gogna (1991a) obtained the dispersion equation for Love waves in a slow elastic layer overlying a saturated porous half space. Tajuddin (1991) investigated dynamic interaction of a saturated porous medium and an elastic half space.

\subsection{Applicability of Biot's theory}

Existence, uniqueness, and regularity of the solution of Biot's equations were presented by Santos (1986). The applicability of the Biot theory has been investigated by Hovem and Ingram (1979), Hovem (1980), and Ogushwitz (1985) for various types of porous media with a wide range of porosity. Ogushwitz (1985) determined that the Biot's theory predicts compressional and shear wave speeds within 3\% for a porous sintered glass saturated with water (Plona, 1980), 1\% for Berea Sandstone, $5 \%$ for Bedford limestone saturated with brine, $8 \%$ for water saturated Bedford limestone, 25 to $30 \%$ for water saturated Massilon sandstone. The last one could be an indication of water sensitivity of sandstone which might reduce the shear modules of the solid matrix due to release of colloidal particles. All these materials represent low-porosity porous media. For suspensions which represent the other end of porosity spectrum, the Biot model agrees well with the experimental data. For porous medium with mid-range porosity values, the Biot's theory matched within 3\% for Ottawa sandpack, $10 \%$ for glass bead pack. Ogushwitz's (1985) work is similar to that of Hovem (1980) except that a theoretically derived structure factor was employed instead of an experimental value. Stoll and 
Bryan (1970) and Stoll $(1974,1977)$ demonstrated the applicability of the Biot theory to marine sediments. Berryman (1980a) gave supporting theoretical evidence to Plona's (1980) experimental data for the dilational waves of the second kind with the identification of coefficients given by Geertsma (1957), Biot and Willis (1957), and Stoll (1979). Plona and Johnson (1984) also provided experimental data verifying Biot's theory. Salin and Schon (1981) provided data for ultrasonic pulse propagation in packed glass beads. Holland and Brunson (1988) examined the Biot's theory as implemented by Stoll for accuracy for a variety of marine sediments. Out of 13 inputs needed, 10 of them were derived empirically and the other 3 were measured. Comparison of predicted and measured values of compressional velocity, attenuation and shear velocity showed excellent agreement. Beebe et al. (1982) compared the predictions of compressional attenuation to estimate velocities and showed good agreement between the predictions and measurements. Berryman (1986a,b) notes that Biot's theory was not successful at predicting the magnitude of the attenuation coefficient in the low-frequency (1$100 \mathrm{~Hz}$ ) range (Murphy, 1982, 1984; Mochizuki, 1982). Berryman (1986b, 1988) attributed this to inhomogeneities in fluid permeability of porous geologic materials and showed that local flow effects dominate the wave attenuation. Johnson (1982) applied Biot's theory to acoustic wave propagation in snow, and obtained data for the $200-800 \mathrm{~Hz}$ frequency range.

As a criticism of Biot's formulation, Rice and Cleary (1976) noted that deadend pores in a porous medium may be sealed off from fully interconnected pores. These dead end pores would not contribute to momentum transfer between the solid and the fluid. Instead, the "closed-off" pores would induce an apparent viscoelastic effect. Levy (1979) observed the effect of unconnected fluid in a similar way. We must point out that this can be avoided by using the "effective porosity" concept and modifying the stress-strain relations for any possible viscoelastic behavior due to dead-end pores. At this point we should note that pore crushing was not taken into account in Biot's theory. In dry porous materials, such a phenomenon can occur and must be included in the formulation (e.g., Carroll and Holt, 1972; Butcher et al., 1974).

Burridge and Keller (1981) provided theoretical justification for Biot's equations by considering the microstructure of porous media. They assumed the scale of the pores to be small in comparison to the macroscopic scale, so that the "two-space method of homogenization" can be used to obtain macroscopic equations. When a dimensionless fluid viscosity term is small, the resulting equations reduce to that of Biot. When the dimensionless viscosity is equal to unity, the equation of a viscoelastic solid is obtained. Pride et. al. (1992) rederived Biot's constitutive relations and obtained the same expressions for the coefficients by using the local volume averaging technique.

\section{Solutions of Biot's formulation}

A number of researchers in various fields solved Biot's formulation either numerically or analytically. In this section, we will review some of these solutions and point out some interesting results. 


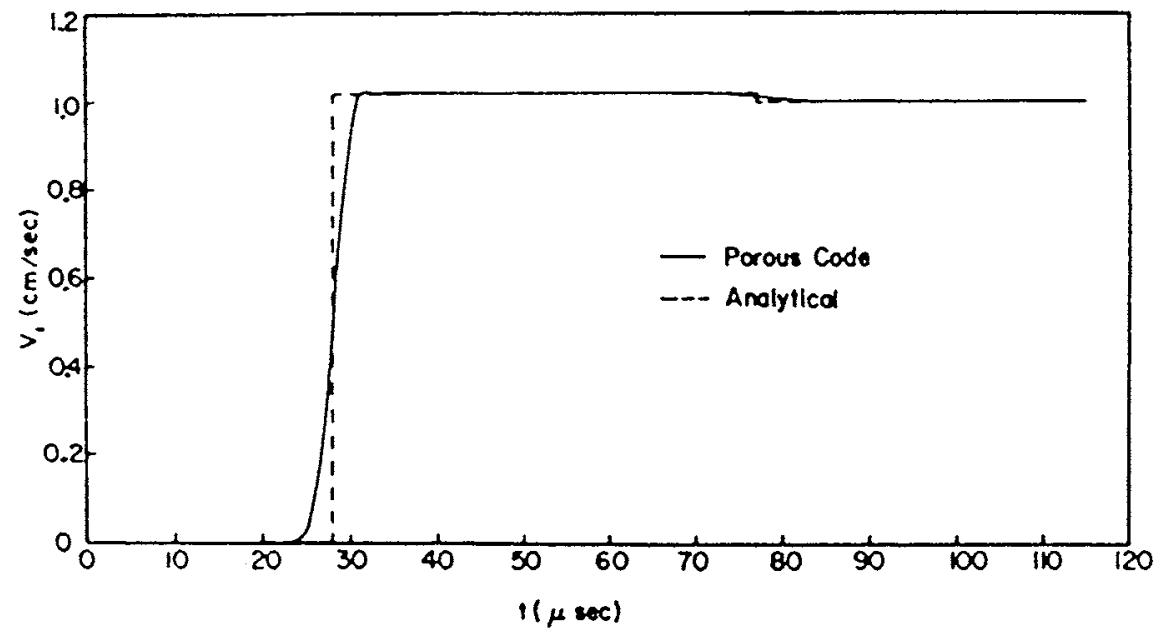

Fig. 7. Solid particle velocity history at $10 \mathrm{~cm}$ with $b=0.219 \times 10^{2} \mathrm{~g} / \mathrm{cm}^{3} \mathrm{sec}$ (after Garg et al., 1974).

\subsection{Analytical solutions of Biot's formulation}

Among various solutions obtained within last two decades, (e.g., Wijesinghe and Kingsbury's $(1979,1980)$ solution with no coupling, i.e., $b=0$, for a harmonic loading of saturated porous layer or Cleary's (1977) solution without inertia, or Verruijt's (1982) solution for cyclic sea wave generated pore pressures), Garg and his coworkers' analytical solution (Garg et al., 1974) is an important one. Garg et al. solved Biot's equations for dilatational waves in terms of displacements $u_{x}$ and $U_{x}$ [equations (2.25) and (2.28)] for a one-dimensional column. They assumed $\rho_{12}=0$. The column is initially at rest and is subjected to a time-dependent disturbance at time zero at the free boundary, $x=0$. They assumed solutions of the form

$$
V_{j}=A_{j} e^{i(\beta x-\theta t)}, \quad j=1,2
$$

$V_{j}$ is the velocity. Subscripts 1 and 2 refer to solid and fluid, respectively. $\beta, \theta$, and $A_{j}$ denote the complex wave number, the circular frequency and wave amplitude, respectively. The phase velocity is obtained by dividing $\theta$ by the real part of $\beta$. The imaginary part of $\beta$ is an attenuation coefficient. Garg et al. took the Laplace transform of equations (2.25), (2.28), and (3.1) and obtained characteristic equation for two extreme cases of weak and viscous coupling, i.e., for small values of $b$ (weak coupling), and for $b \rightarrow \infty$ (strong viscous coupling). They obtained the exact inverse Laplace transformation after making some simplifying assumptions. Hong et al. (1988) has compared Garg et al.'s solutions with solutions obtained by numerical inverses of the Laplace transformed solutions with no approximations. They found that the difference is insignificant. Garg et al. stated that strong viscous coupling causes the two wave fronts to merge to a single front and the porous medium behaves like a single medium with bulk properties. They presented finite difference solutions for the general case with no assumptions on viscous coupling.

Figures 7 and 8 show propagation of two fronts when the viscous coupling is 


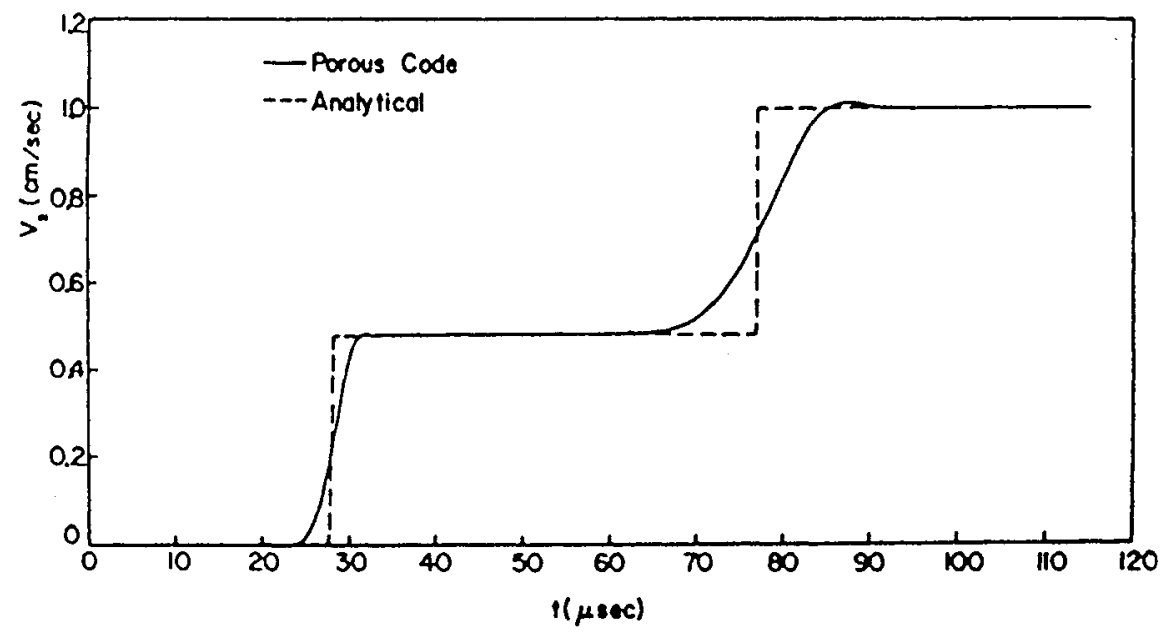

Fig. 8. Fluid velocity history at $10 \mathrm{~cm}$ with $b=0.219 \times 10^{2} \mathrm{~g} / \mathrm{gm}^{3} \mathrm{sec}$ (after Garg et al., 1974).

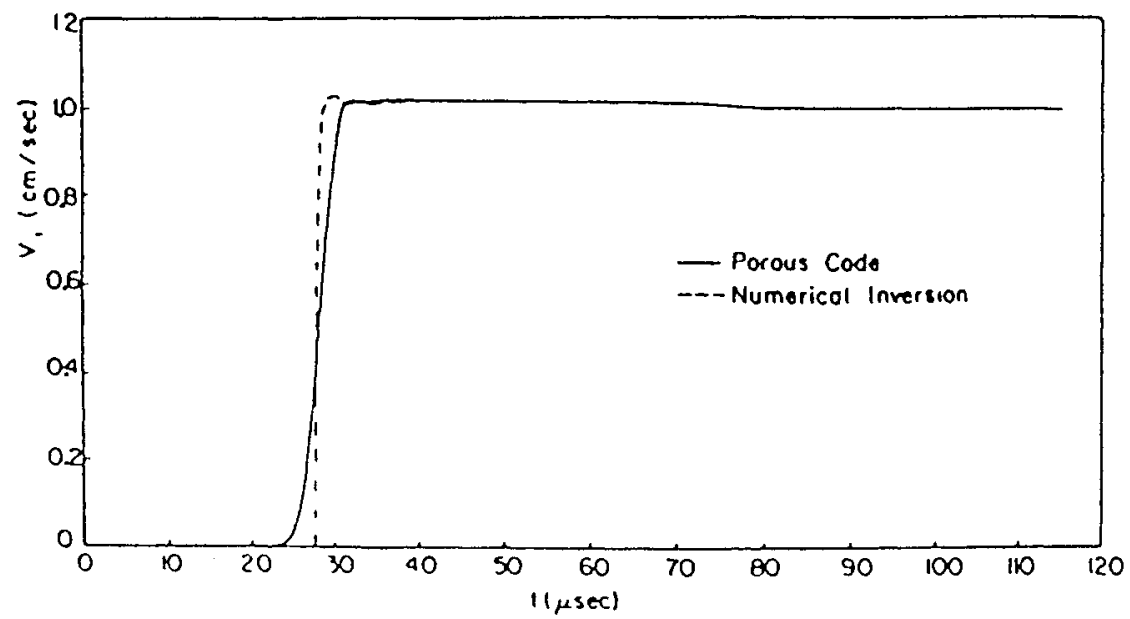

Fig. 9. Solid particle velocity history at $10 \mathrm{~cm}$ with $b=0.219 \times 10^{4} \mathrm{~g} / \mathrm{cm}^{3} \mathrm{sec}$ (after Garg et al., 1974).

weak $\left(b=21.9 \mathrm{~g} / \mathrm{cm}^{3} \mathrm{sec}\right.$.). For moderate viscous coupling $\left(b=2190 \mathrm{~g} / \mathrm{cm}^{3} \mathrm{sec}\right.$.), we also observe two waves. However as seen in Fig. 10 wave velocity in the fluid phase gradually increases between the two fronts. This gradual increase is due to viscous momentum transfer between the solid and fluid phases. Figure 11 shows that in the far field (distant to the boundary at $x=0$ ), the wave of second kind disappears and becomes a standing wave with time. Garg et al. noted that oscillations in Fig. 11 at the head of the pulse are due to numerical approximations. For strong viscous coupling $\left(b=2190 \times 10^{4} \mathrm{~g} / \mathrm{cm}^{3} \mathrm{sec}\right.$.), two wave fronts merge into one (Fig. 12). We should note that for in all figures the numerical solutions cause the wave front(s) to smear. Yew and Jogi (1976) also obtained a solution by Laplace transformation similar to Garg et al's. (1974). Jones (1969) studied the propagation of a pulse wave in porous media. 


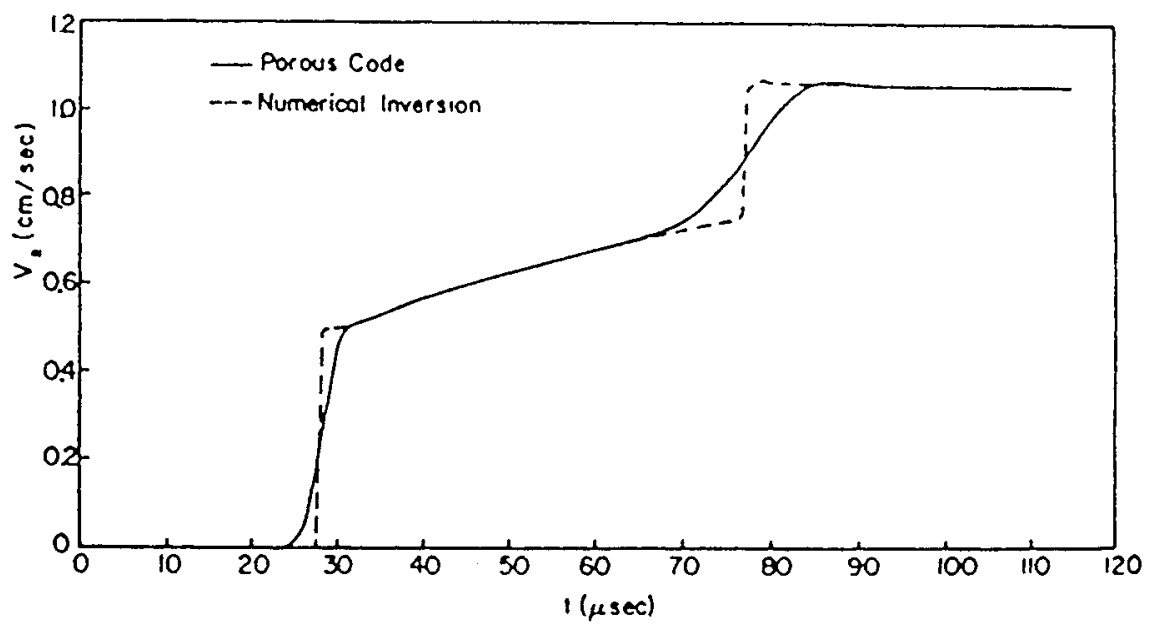

Fig. 10. Fluid velocity history at $10 \mathrm{~cm}$ with $b=0.219 \times 10^{4} \mathrm{~g} / \mathrm{cm}^{3} \mathrm{sec}$ (after Garg et al., 1974).

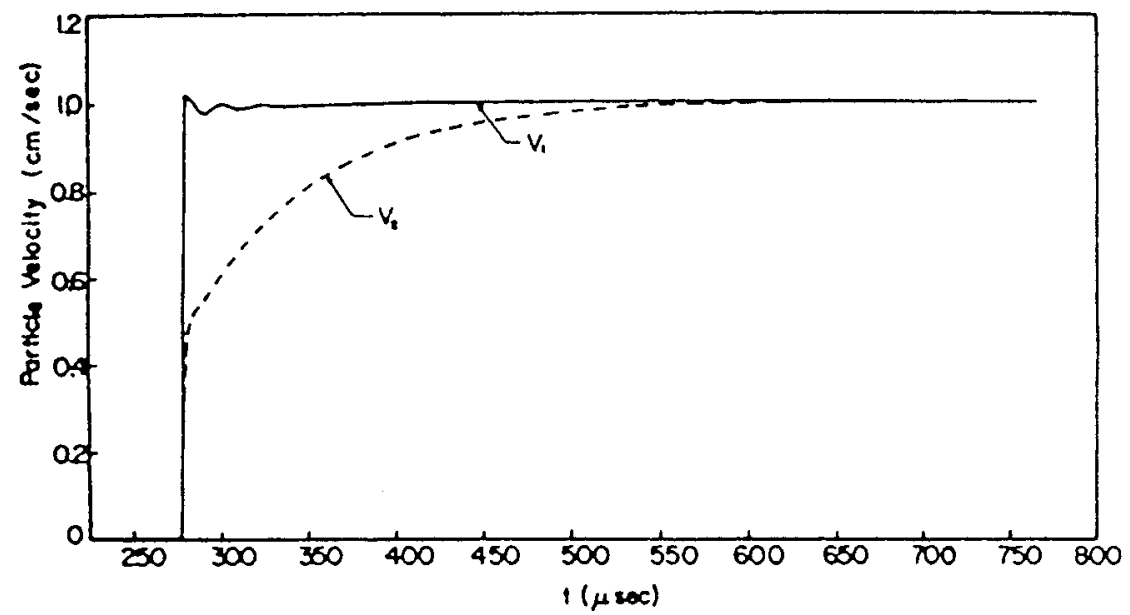

Fig. 11. Solid and fluid velocities at $100 \mathrm{~cm}$ with $b=0.219 \times 10^{4} \mathrm{~g} / \mathrm{cm}^{3} \mathrm{sec}$, obtained by numerical inversion (after Garg et al., 1974).

Burridge and Vargas (1979) obtained analytical solutions for $P$ and $S$ waves due to an instantaneous point body force acting in a uniform whole space. Biot's equations [equations (2.48) and (2.49)] have been solved by introducing four scalar potentials to decouple the system of equations, and transforming them to symmetric hyperbolic systems to be solved by Laplace transformation. It has been found that $P$ and $S$ waves have the shape of a Gaussian instead of a sharp pulse shape. Norris (1985) derived the time harmonic Green function of Biot's equations for a point load in an infinite saturated porous medium. He obtained the solutions for rotational waves as well as compressional waves. As Burridge and Vargas (1979) did, Norris observed that Gaussian shaped pulses broaden with time and distance. The integral representation of displacement fields and pore pressure was 


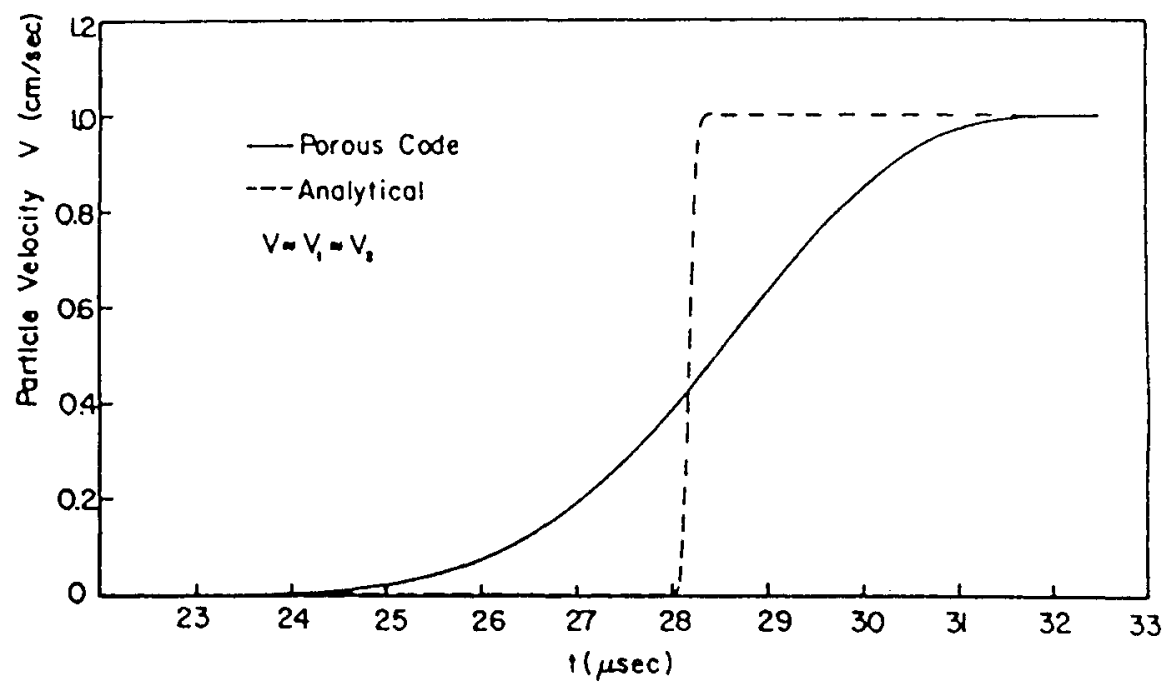

Fig. 12. Velocity histories at $10 \mathrm{~cm}$ with $b=0.219 \times 10^{8} \mathrm{~g} / \mathrm{cm}^{3} \mathrm{sec}$ (after Garg et al., 1974).

also suggested by Predeleanu (1984). Boutin et al. (1987) presented a new analytical formulation of Green's function. Their solution is valid at any frequency range. Parra (1991) developed an analytical solution for seismic wave propagation associated with a point source in a stratified saturated porous medium.

Based on the construction of synthetic seismograms, Boutin et al. concluded that the signal wave form is strongly dependent on the permeability value, thus raising the possibility of determining the permeability values from seismic explorations (see Section 6 for details). Bonnet (1987) provided an harmonic solution by an analogy with a thermoelasticity problem.

\subsection{Numerical solutions}

During the last fifteen years, the numerical solution of Biot's wave propagation equations on large scale computers have gained popularity due to ability to solve a large number of equations in a multi-dimensional space. Among many other studies, a finite element solution by Ghaboussi and Wilson (1972) appears to be one of the early studies. Ghaboussi and Wilson's formulation which is a generalization of Sandhu and Pister's (1970) technique, used the displacement of the solid, $\boldsymbol{u}$, and relative fluid displacement $(\boldsymbol{U}-\boldsymbol{u})$ as two field variables [equations (2.23) and (2.24)]. Ghaboussi and Wilson calculated the fluid pressure, $p$, from the volumetric changes of the solid and fluid through stress-strain relations. Differential equations were transformed by using the "Galerkin process of weighted residuals" to functional forms which are discretized by the finite element method. Step loading applied to half-space of saturated elastic porous solid was given as an example. Galerkin solution was also employed by Santos et al. (1986).

Later, Sandhu et al. (1989) presented a mixed variational formulation taking the soil displacement, relative fluid displacement, and fluid pressure as three field variables, as a special case of general variational principle of Sandhu and Hong 
(1987). By taking pore pressure as a variable, a continuous solution for pressure has been obtained. Numerical solutions were compared with Garg et al.'s (1974) analytical solution for a special case.

Hiremath et al. (1988), Morland et al. (1987, 1988) solved Biot's equations for a one-dimensional case by employing the Laplace transformation and numerical inversion. These results were compared with a finite element solution. It has been concluded that numerical solutions compare favorably with the Laplace solutions for weak as well as strong viscous coupling.

Zienkiewicz and his co-workers obtained various finite element solutions for simplified Biot theory under transient conditions. Among various assumptions made, drained or undrained behavior depending on the permeability of the porous medium and rapidity of loading are the dominating factors to characterize a particular problem. For example, an earthquake which can be modeled as an impulse loading can be investigated as a completely undrained behavior if the permeability of the soil is not high. Similar assumptions have been also made by other researchers to study the effect of water waves on sea beds (Mei and Foda, 1981 in Section 7.2). Nur and Booker (1972) suggested that due to the agreement between computed rates of attenuation and observed rates of aftershock activity, aftershocks can be caused by the flow of groundwater due to changes in pore water pressures induced by large shallow earthquakes.

In a series of papers, Zienkiewicz et al. $(1980,1982 \mathrm{a}, \mathrm{c})$ solved the following simplified equations after making further assumptions for a numerical solution

$$
\begin{aligned}
& \nabla \cdot \sigma+\rho g \nabla z=\rho \frac{\partial^{2} u}{\partial t^{2}}+\rho_{\mathrm{f}} \frac{\partial^{2} w}{\partial t^{2}} \\
& -\nabla p+\rho_{\mathrm{f}} g \nabla z=\rho_{\mathrm{f}} \frac{\partial^{2} u}{\partial t^{2}}+\frac{\rho_{\mathrm{f}}}{n} \frac{\partial^{2} w}{\partial t^{2}}+\frac{\rho_{\mathrm{f}} g}{k^{\prime}} \frac{\partial w}{\partial t} \\
& \nabla \cdot \frac{\partial w}{\partial t}=-\frac{\partial \epsilon}{\partial t}-\frac{1-n}{K_{\mathrm{s}}} \frac{\partial p}{\partial t}+\frac{1}{3 K_{\mathrm{s}}} \frac{\partial \sigma^{\prime}}{\partial t}-\frac{n}{K_{\mathrm{f}}} \frac{\partial p}{\partial t}
\end{aligned}
$$

Equations (3.2)-(3.4) are the momentum balance equations for the porous medium, and fluid phase and mass balance equation for the fluid, respectively. $\sigma$ is the total stress tensor. $\sigma^{\prime}$ is the effective stress tensor. $K_{\mathrm{s}}$ and $K_{\mathrm{f}}$ are the bulk modules of the solid grains and the fluid, respectively. The first term on the right hand side of equation (3.4) incorporates the solid matrix compressibility. The second and the third terms represent the rate of pore volume increase due to the increase in pore fluid pressure and effective stress change, respectively. The last term represents the compressibility of pore fluid. In equation (3.3), the term $k^{\prime}$ is the hydraulic conductivity. $\rho$ is the "total density". For a correct interpretation, equations (3.2) and (3.3) should be compared with equations (2.48) and (2.49).

Zienkiewicz and Bettess (1982c) consider a case in which the acceleration in the fluid is neglected. The formulation for this "medium speed phenomena" is referred to as the $u-p$ formulation. If all acceleration terms are neglected, it corresponds to "very slow phenomena" which is the classical consolidation problem in soil mechanics. "Very rapid phenomena" occurs when the permeability 
becomes very small or $w, \partial w / \partial t, \partial^{2} w / \partial t^{2}$ never reach to significant values. This is the "undrained behavior" which is also known as the "penalty type" formulation. The total system can be expressed by omitting the momentum balance equation for fluid (equation (3.3)), thus $u$ becomes the primary variable. "Drained behavior" is another extreme case which occurs when the permeability (or hydraulic conductivity) reaches to infinity. $p$ can be calculated independently, and then $\boldsymbol{u}$ is calculated using the known values of $p$. This extreme case does not occur ever with dynamic effects and it is only possible when all transient behavior ceases.

For one dimensional case (a soil layer with thickness, $L$ ) i.e., $\sigma=\sigma^{\prime}-p, e=$ $\partial u / \partial z$, and $\sigma^{\prime}=D \epsilon$ and neglecting the grain compressibility, two dilatational wave equations in terms of $\boldsymbol{u}$ and $\boldsymbol{U}$ are obtained (similar to equations (2.25) and (2.28) with different mass coefficients). For the periodic case (i.e., $\exp (-i \omega t)$ where $\omega$ is the angular frequency), these equations become

$$
\begin{aligned}
& \frac{\left[D+\frac{K_{\mathrm{f}}}{n}\right]}{\rho} \frac{d^{2} \bar{u}}{d z^{2}}+\frac{K_{\mathrm{f}}}{\rho n} \frac{d^{2} \bar{w}}{d z^{2}}=-\omega^{2} \bar{u}-\omega^{2} \frac{\rho_{\mathrm{f}}}{\rho} \bar{w} \\
& {\left[\frac{d^{2} \bar{u}}{d z^{2}}+\frac{d^{2} \bar{w}}{d z^{2}}\right] \frac{K_{\mathrm{f}}}{\rho_{\mathrm{f}}}=-\omega^{2} n \bar{u}-\omega^{2} \bar{w}+\frac{i \omega n g}{k^{\prime}} \bar{w}}
\end{aligned}
$$

where overbar denotes transformed variables in the Fourier space.

The coefficient of the first term of equation (3.5) is the square of compression wave velocity $V_{\mathrm{c}}=K_{\mathrm{f}} / \rho_{\mathrm{f}}$ is the speed of sound in water. Results of Zienkiewicz et al. (1980) have shown that in the space of two dimensionless parameters $\pi_{1}$ and $\pi_{2}$ which are defined by

$$
\pi_{1}=\frac{k^{\prime} V_{\mathrm{c}}^{2}}{g\left(\rho_{\mathrm{f}} / \rho\right) \omega L^{2}}, \quad \pi_{2}=\frac{\omega^{2} L^{2}}{V_{\mathrm{c}}^{2}}
$$

There are three zones. In zone one, the propagation is slow so that the consolidation problem $(C)$ would solve the problem. In zone II, the $u-p$ approximation $(Z)$ would be satisfactory. Zone III includes extremely rapid motions which can be described by full Biot theory $(B)$ as given by equations (3.2) through (3.4). Figure 13 shows the summary of basic conclusions. They noted the existence of small zones which are drained even when most of the medium is undrained. This "boundary layer" concept was studied by Mei and Foda $(1981,1982)$ (see Section 7.2). Zienkiewicz et al. (1982b) applied $u-p$ model to analyze the earthquake problem by neglecting the coupling acceleration term. They employed various plastic constitutive equations to represent the soil deformation.

Later Zienkiewicz and Shiomi (1984) added the convective fluid acceleration term $\left[\rho_{\mathrm{f}}(\partial w / \partial t \nabla . \partial w / \partial t) / n\right]$ to the right hand side of equation (3.3). Similar adjustment was also introduced into equation (3.2).

Prevost $(1982,1984)$ solved the coupled equations of mass and momentum balance by using a finite element technique. Time integration is handled by an implicit/explicit predictor/multicorrector scheme. The method has been applied to one- and two-dimensional initial value problems. Later, Prevost (1985) allowed 


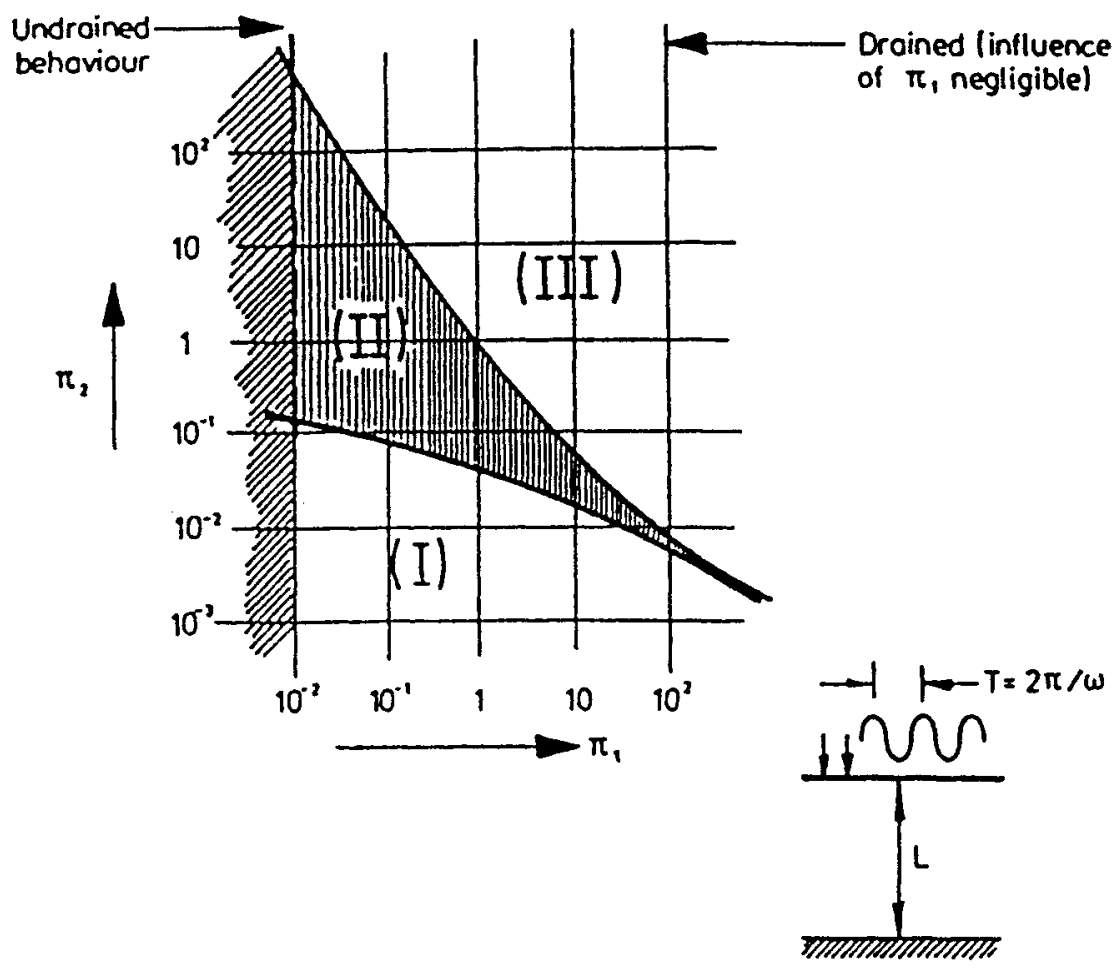

Fig. 13. Zones of applicability of various assumptions (after Zienkiewicz et al. (1980)). Zone 1: $B=$ $Z=C$. Slow phenomena $\left(\partial^{2} U / \partial t^{2}\right.$ and $\partial^{2} u / \partial t^{2}$ can be neglected). Zone $2: B=Z \neq C$. Moderate speed $\left(\partial^{2} U / \partial t^{2}\right.$ can be neglected. Zone $3: B \neq Z \neq C$. Fast phenomena $\left(\partial^{2} U / \partial t^{2}\right.$ can not be neglected), only full Biot equation [Equations (3.2) through (3.4)] valid.

the compressibility of fluid by treating the fluid contributions to the equations of momentum balance implicitly. This approach removed the restriction on the time step size. Halpern and Christiano $(1986 \mathrm{a}, \mathrm{b})$ applied Biot's formulation to analyze various foundation problems.

Hassanzadeh (1991) presented an acoustic modelling method that involves numerical simulation of two-dimensional low frequency transient wave propagation. The method is based on explicit finite difference formulation of Biot's system of equations. Zhu and Mcmechan (1991) developed a two-dimensional finite difference method allowing investigation of spatial variations in porosity, permeability and fluid viscosity.

Bougacha and Tassoulas (1991) used the finite element method to analyze damreservoir-sediment-foundation interaction. They modelled the sediment by using Biot's formulation for saturated porous medium. Bougacha et al. (1993a) developed a spatially semi-discrete finite element technique for layered, saturated porous medium. Bougacha et al. (1993b) applied the formulation to calculate dynamic stiffness of strip and circular foundations. Chang et al. (1991) presented a singular integral solution technique for solving dynamic problems. They also 
showed an analogy between thermoelasticity and dynamic poroelasticity in the frequency domain.

\subsection{Solutions by the method of characteristics}

The method of characteristics have been used widely to solve hyperbolic partial differential equations. By using the method characteristics, partial differential equations are transformed into time-dependent ordinary differential equations. These canonical equations are solved along the characteristic lines.

Streeter et al. (1974) applied the characteristics method to study the wave propagation in a layered soil due to earthquakes. Streeter et al. presented equation (2.23) in a form

$$
\frac{\partial \tau_{x z}}{\partial z}-\rho \frac{\partial^{2} u_{x}}{\partial t^{2}}=\frac{\partial \tau_{x z}}{\partial z}-\rho \frac{\partial v_{x}}{\partial t}=0
$$

for a one-dimensional (vertical) soil column. $\rho$ is the density of the soil. The displacement in the vertical direction is zero. Shearing stresses are set up by horizontal motions imposed at the base of the column (i.e., earthquake) and they travel in the vertical direction.

The soil is modeled as a viscoelastic solid with a constitutive equation

$$
\tau_{x z}=G \frac{\partial u_{x}}{\partial z}+\mu_{\mathrm{s}} \frac{\partial^{2} u_{x}}{\partial z \partial t}
$$

where $G$ is the shear modulus and $\mu_{\mathrm{s}}$ is the viscosity of soil. Differentiation of equation (3.9) gives

$$
\frac{\partial \tau_{x z}}{\partial t}=G \frac{\partial v_{x}}{\partial z}+\mu_{\mathrm{s}} \frac{\partial^{2} v_{x}}{\partial z \partial t}
$$

If the time derivative in equation (3.10) is approximated by finite difference equation, and then combined with equation (3.8) after multiplying with an unknown multiplier $\theta$, to give

$$
\left[\theta \frac{\partial \tau_{x z}}{\partial z}+\frac{\partial \tau_{x z}}{\partial t}\right]-\theta \rho\left[\frac{\partial v_{x}}{\partial z}\left(g+\frac{\mu_{\mathrm{s}}}{\Delta t}\right) \frac{1}{\theta \rho}+\frac{\partial v_{x}}{\partial t}\right]+\frac{\mu_{\mathrm{s}}}{\Delta t}\left(\frac{\partial v_{x}}{\partial z}\right)_{\mathrm{c}}=0
$$

where the subscript $c$ represents the value determined at point $c$ on the $z-t$ space. Partial derivatives in equation (3.11) are expressed in terms of total derivatives as

$$
\frac{d \tau_{x z}}{d t}-\rho V_{\mathrm{s}} \frac{d v_{x}}{d t}+\frac{\mu_{\mathrm{s}}}{\Delta t}\left(\frac{\partial v_{x}}{\partial z}\right)=0
$$

when 


$$
\frac{d z}{d t}=\theta=\frac{1}{\theta \rho}\left(G+\frac{\mu_{\mathrm{s}}}{\Delta t}\right)
$$

Equation (3.13) is solved for

$$
\frac{d z}{d t}=\theta= \pm\left(\frac{G}{\rho}+\frac{\mu_{\mathrm{s}}}{\rho \Delta t}\right)^{1 / 2}= \pm V_{\mathrm{s}}
$$

where $V_{\mathrm{s}}$ is the shear velocity. Equations (3.12) and (3.14) give four ordinary equations to be solved, replacing two partial differential equations [equations (3.8) and (3.10)]. One-dimensional pressure wave propagation is similar to shear wave propagation except that the velocity of the compression wave is given by

$$
V_{\mathrm{p}}= \pm\left(\frac{K_{\mathrm{s}}}{\rho}+\frac{\mu_{\mathrm{s}}}{\rho \Delta t}\right)^{1 / 2}
$$

where $K_{\mathrm{s}}$ is the bulk modulus of the soil.

Propagation of pore pressure and water flux are analyzed by simultaneous solution of momentum and mass balance equations

$$
\begin{aligned}
& K \frac{\partial h}{\partial z}+\frac{K r^{2}}{g} \frac{\partial V_{z}}{\partial t}+V_{z}=0 \\
& \frac{\partial h}{\partial t}+\frac{V_{\mathrm{p}}^{2}}{g} \frac{\partial V_{z}}{\partial z}-\frac{V_{\mathrm{P}}^{2}}{g} \frac{1}{1+e} \frac{\Delta e}{\Delta t}=0
\end{aligned}
$$

where $e$ is the void ratio $(n /(1-n))$. Streeter et al. (1974) present various examples including the liquefaction of an earth dam. $r$ is an inertia multiplier.

Van der Grinten et al. (1985) solved the conservation of mass and momentum equations for a saturated porous medium

$$
\begin{aligned}
& \frac{\partial V_{x}}{\partial x}+\frac{1-n}{n} \frac{\partial v_{x}}{\partial x}=-\frac{1}{K_{\mathrm{f}}} \frac{\partial p}{\partial x} \\
& \frac{\partial v_{x}}{\partial x}=\frac{1}{K_{\mathrm{s}}} \frac{\partial \sigma^{\prime}}{\partial t} \\
& {\left[n \rho_{\mathrm{f}}+\left(\alpha^{* *}-1\right) n \rho_{\mathrm{f}}\right] \frac{\partial V_{x}}{\partial t}-\left(\alpha^{* *}-1\right) n \rho_{\mathrm{f}} \frac{\partial \nu_{x}}{\partial t}=-n \frac{\partial p}{\partial x}+n \rho_{\mathrm{f}} g} \\
& +n^{2} \mu_{\mathrm{f}} a^{\prime}\left(v_{x}-V_{x}\right) \\
& {\left[(1-n) \rho_{\mathrm{s}}+\left(\alpha^{* *}-1\right) n \rho_{\mathrm{f}}\right] \frac{\partial v_{x}}{\partial t}-\left(\alpha^{* *}-1\right) n \rho_{\mathrm{f}} \frac{\partial V_{x}}{\partial t}=} \\
& -\frac{\partial \sigma^{\prime}}{\partial x}-(1-n) \frac{\partial p}{\partial x}+(1-n) \rho_{\mathrm{s}} g-n^{2} \mu_{\mathrm{f}} a^{\prime}\left(v_{x}-V_{x}\right)
\end{aligned}
$$

where $\rho_{\mathrm{s}}$ is the density of solid. The term $\left(\alpha^{* *}-1\right) n \rho_{\mathrm{f}}$ represents the mass coupling between the fluid and the solid matrix. The added mass parameter $\alpha^{* *}$ 
depends on the structure of the porous matrix (Johnson et al., 1982). Equations (3.18)-(3.21) were first presented by de Josselin de Jong (1956). Equations (3.20) and (3.21) can directly be obtained from Biot's equations [equations (2.23) and (2.24)]. Equation (3.18) is the mass balance equation for the fluid after some mathematical manipulations (Bear and Corapcioglu, 1981) and equation (3.19) is the elastic stress-strain relation for the solid matrix. By applying the method of characteristics, equations (3.18)-(3.21) are obtained in characteristic form

$$
\left(\frac{\partial}{\partial t}+V_{\mathrm{p}} \frac{\partial}{\partial x}\right)\left(A \sigma^{\prime}+B p+C v_{x}+D V_{x}\right)=E\left(v_{x}-V_{x}\right)
$$

$V_{\mathrm{p}}$ is obtained from

$$
F V_{\mathrm{p}}^{4}+H V_{\mathrm{p}}^{2}+1=0
$$

where $A, B, C, D, E, F$ and $H$ are parameters in terms of equation coefficients. When the pore fluid is air, the compressibility of the matrix is much smaller than that of air. Therefore, the porous medium can be considered rigid. Since interactive forces are much larger than inertial forces, equations are decoupled. The momentum equation will reduce to Forcheimer equation by adding a term proportional to the velocity squared (see equation (6.11)). Then the governing equations reduce to

$$
\begin{aligned}
& \frac{\partial \rho_{\mathrm{a}}}{\partial t}+\frac{\partial\left(\rho_{\mathrm{a}} V_{\mathrm{a}}\right)}{\partial x}=0 \\
& \frac{\partial p_{\mathrm{a}}}{\partial x}=-n a^{\prime} \mu_{\mathrm{f}} V_{\mathrm{a}}-n^{2} b^{\prime} \rho_{\mathrm{a}} V_{\mathrm{a}}\left|V_{\mathrm{a}}\right|
\end{aligned}
$$

where $V_{\mathrm{a}}$ is the air velocity, $p_{\mathrm{a}}$ is the air pressure, $\rho_{\mathrm{a}}$ is the density of the air, and $a^{\prime}$ and $b^{\prime}$ are Forcheimer coefficients. For an isothermal compression

$$
\frac{d \rho_{\mathrm{a}}}{\rho_{\mathrm{a}}}=\frac{d p_{\mathrm{a}}}{p_{\mathrm{a}}}
$$

when the solid matrix is filled by air instead of water, the wave is strongly damped, and the permeability is not frequency dependent. As concluded by other studies, when the pore fluid is water, the permeability is strongly dependent on frequency due to viscosity. In a dry porous medium, the dilatational wave of the second kind which is strongly attenuated is the only wave observed in the pores. Furthermore, it is determined that transient permeability is approximately one-third of the stationary value. The contribution of added mass which is neglected by most researchers (e.g., Garg et al., 1974; Mei and Foda, 1981) was found to be significant. Later, van der Grinten et al., (1987a) provided new experimental evidence by measuring pore pressures and strain simultaneously. They concluded that the behavior of the wave of the second kind is affected by the boundary conditions at the top of the solid matrix. The influence of boundary conditions is also discussed by Geertsma and Smit (1961) and Zolotarjew and Nikolaevskij (1965). Van der 
Grinten et al. (1987a) used approximations of frequency correction factor [see equations (2.44) and (7.10)] for low and high frequencies, respectively.

$$
\begin{aligned}
& F(\kappa)=1+i\left(\frac{\kappa^{2}}{24}\right) \quad \text { as } \quad \kappa \rightarrow 0 \\
& F(\kappa)=[(1+i) / 4 \sqrt{2}] \kappa \quad \text { as } \quad \kappa \rightarrow \infty
\end{aligned}
$$

where $\kappa$ is the transient Reynolds number defined as

$$
\kappa=\operatorname{Re}=R_{\mathrm{p}} \sqrt{\frac{\omega}{v_{\mathrm{f}}}}
$$

where $R_{\mathrm{p}}$ is the radius of cylinders of the cylindrical duct model representing the porous medium. The reader should compare the definition of Reynold's number given here with equation (6.10) given by Geertsma (1974). Later, van der Grinten et al. (1987b), extended their analysis to partially saturated medium by varying the bulk modulus of fluid. The reader is referred to Section 5 for a review of this type of treatment to model unsaturated porous medium.

\section{Liquefaction of soils}

When loose saturated sands are subjected to vibrations, their porosity decreases. If the pore water pressure increases due to lack of drainage, the effective stress vanishes when the pore pressure reaches the overburden pressure (total stress) with continuous vibration. This can be stated by the effective stress principle

$$
\sigma=\sigma^{\prime}-p I
$$

where $\sigma$ is the overburden pressure, $\sigma^{\prime}$ is the effective stress, $I$ is the unit tensor, and $p$ is the pore pressure. At this point, the sand looses its shear strength and behaves like a liquid. When this happens, the soil cannot support the weight of the structure resting on it. Structures sink into the soil as observed in Niigata (Japan) earthquake of 1964. This phenomenon is known as liquefaction in soil mechanics (Scott, 1986). Liquefaction can be observed even several hours after the initial shock. Since this is a problem of great practical importance, quite a number of studies tried to predict the liquefaction potential of soils.

In liquefaction studies, the soil is represented by one or more layers with homogeneous properties resting on a solid rock base. The earthquake excitation is at the base and resulting shear waves propagate vertically upward through the soil column. Shear stresses induced by the earthquake are approximated by cyclic horizontal shear stresses applied at the base. Since, liquefaction is caused by pore pressure increase, the pore pressure dissipation during and after a period of cyclic loading, needs to be calculated. A similar phenomenon can occur in sea-bed deposits of sand subjected to storm-wave loadings. The concept of pore pressure generation under cyclic loading condition was first introduced by Seed and his coworkers in various publications (e.g., DeAlba et al., 1976; Martin et al., 1975; 


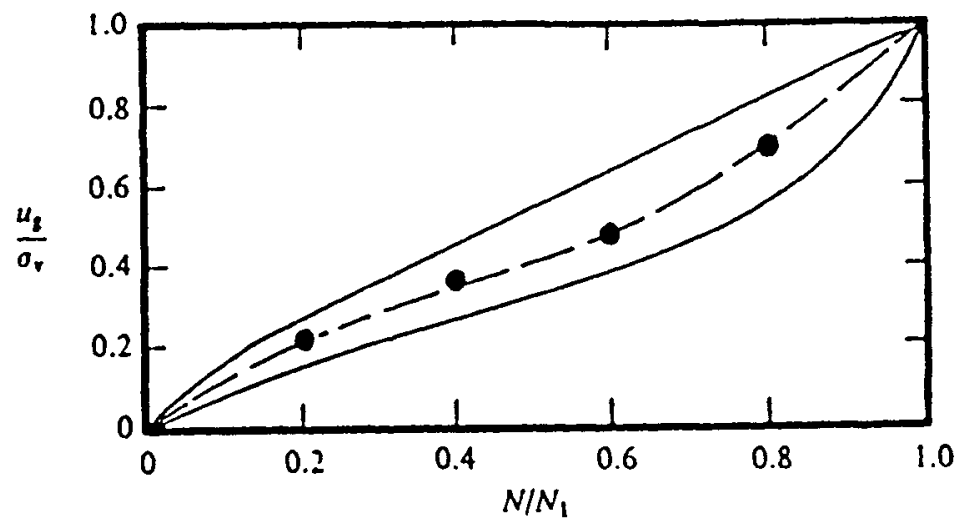

Fig. 14. Rate of pore water pressure buildup in cyclic simple shear test (after Seed and Brooker, 1977).

Rahman et al., 1977; Seed et al., 1976; Seed and Rahman, 1978) and outlined in Seed and Idriss (1982). Such an approach is known as "effective stress method." Seed and his coworkers have found that pore pressure generation in a cyclic undrained simple shear test falls within a narrow range as shown in Fig. 14. The average curve can be approximated by

$$
r_{\mathrm{p}}=\frac{p_{\mathrm{g}}}{\sigma_{\mathrm{o}}^{\prime}}=\frac{2}{\pi} \sin ^{-1}\left(\frac{N}{N_{\mathrm{l}}}\right)^{1 / 2 \theta}
$$

where $N$ is the number of stress cycles applied, $N_{\mathrm{M}}$ is the number of stress cycles needed for initial liquefaction, and $j=0.7$. $\sigma_{o}^{\prime}$ is the initial vertical effective stress. $p_{\mathrm{g}}$ is the generated pore pressure. Then, the rate of pore pressure generation is obtained from equation (4.1) as

$$
\frac{\partial p_{\mathrm{g}}}{\partial t}=\frac{\partial p_{\mathrm{g}}}{\partial N} \frac{\partial N}{\partial t}=\frac{\sigma_{\mathrm{o}}^{\prime}}{\theta \pi T_{\mathrm{D}}}\left(\frac{N_{\mathrm{eq}}}{N_{\mathrm{L}}}\right) \frac{1}{\sin ^{2 \theta-1}\left(\pi r_{\mathrm{p}} / 2\right) \cos \left(\pi r_{\mathrm{p}} / 2\right)}
$$

Note that in equation (4.2), irregular cyclic loading is converted to an equivalent number of uniform stress cycles, $N_{\text {eq }}$ occurring in a time span $T_{\mathrm{D}}$ by $\partial N / \partial t=$ $N_{\text {eq }} / T_{\mathrm{D}}$. Then, combined pore pressure generation and dissipation is obtained from the solution of

$$
\frac{\partial p}{\partial t}=\frac{C_{\mathrm{v}}}{r} \frac{\partial}{\partial r}\left(r \frac{\partial p}{\partial r}\right)+C_{\mathrm{v}} \frac{\partial^{2} p}{\partial z^{2}}+\frac{\partial p_{\mathrm{g}}}{\partial t}
$$

where $C_{\mathrm{v}}$ is the consolidation coefficient and $r$ and $z$ are the radial and vertical coordinates. An example given by Seed et al., 1976 (Fig. 15a) shows that the sand layer at a depth of $15 \mathrm{ft}$ liquefies after about 21 seconds of shaking during the earthquake. Liquefied condition propagates to $40 \mathrm{ft}$ at 40 seconds (Fig. 15a). After earthquake stops at 50 seconds, pore pressures below $15 \mathrm{ft}$ dissipate. However, pore pressures above $15 \mathrm{ft}$ continue to build up and after about $12 \mathrm{~min}$., the water in the top foot would flow from the ground (Fig. 15b). Seed et al., noted that 


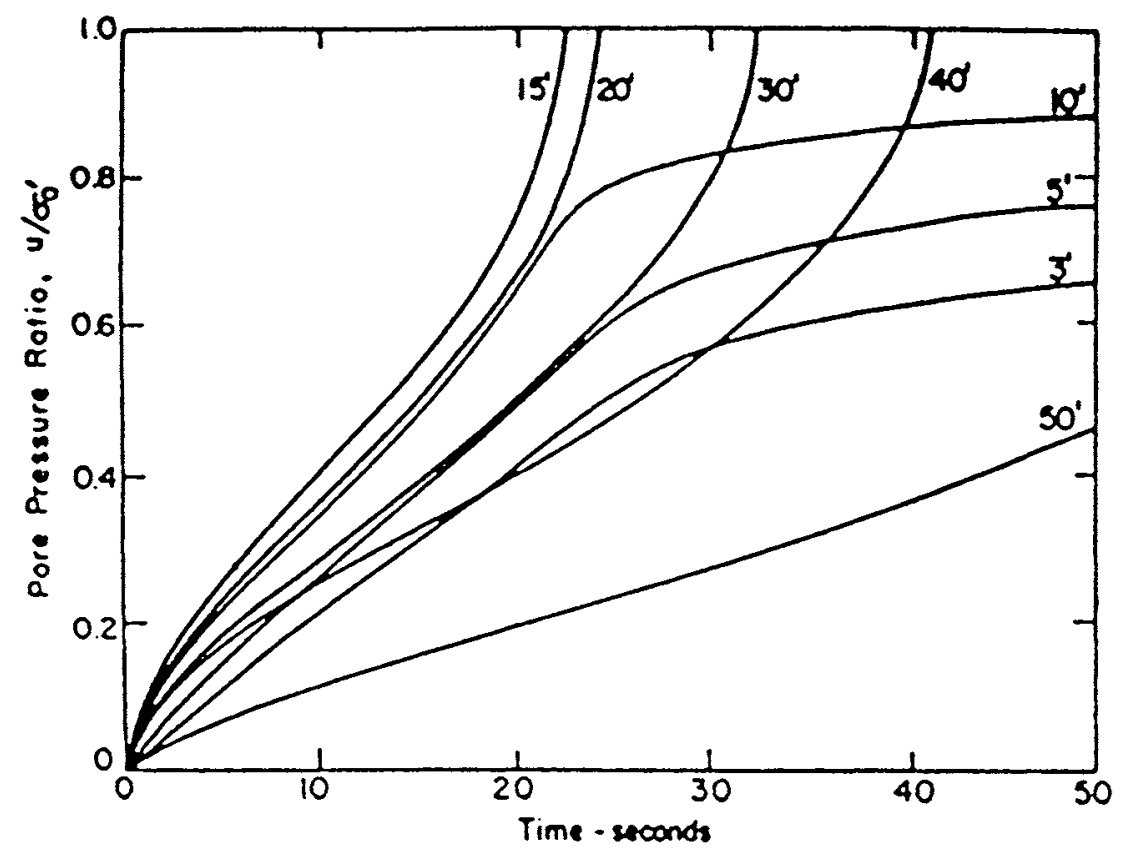

Fig. 15A. Computed development of pore water pressures during earthquake shaking (after Seed et al., 1976).

lower water table would decrease the liquefaction potential. Seed and Idriss (1982) noted that a more fundamental approach by Finn et al. (1977) shows only small differences in results.

Finn et al. $(1976,1977)$ developed a non-linear method of analysis of liquefaction in which the momentum balance equations was coupled by the pore water pressure generation model given by equation (4.3). Later, a more general approach by solving Biot equations were presented by Ishihara and Towhata (1982). Finn et al.'s (1976) stress-strain relations were used by Mansouri et al. (1983) to study the liquefaction potential of an earth dam.

Streeter et al. (1974) presented a characteristics method which treated responses of the pore water and the solid matrix separately as uncoupled problems. Pore pressures were introduced by defining volume changes. The details of Streeter et al.'s technique are given in Section 3.4 (equations (3.8)-(3.17)). Later Liou et al. (1977) developed a liquefaction analysis of saturated sands. They studied the propagation of shear and pressure induced by the earthquake motion at the base of the sand deposit. Liou et al.'s shear wave submodel is similar to that of Streeter et al.'s except the coefficient of viscosity in equation (3.9). Pressure wave submodel consists of momentum balance equation for the solid

$$
\frac{\partial \sigma}{\partial t}+\rho g \nabla z=\rho \frac{\partial v}{\partial t}+n \rho_{\mathrm{f}} \frac{\partial V_{\mathrm{r}}}{\partial t}
$$

momentum balance equation for the pore water, 


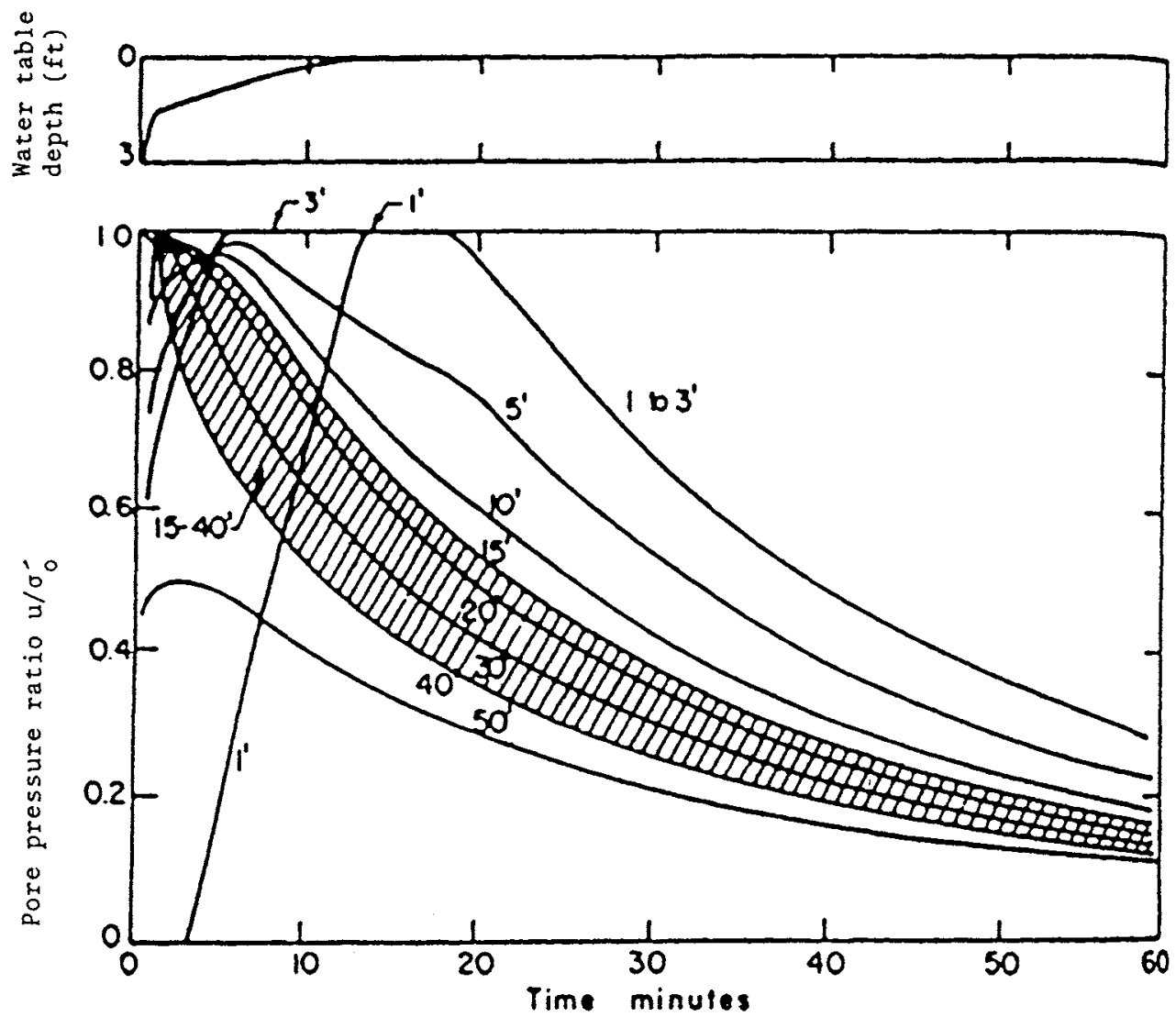

Fig. 15B. Computed variation of pore water pressures in 60-minute period following earthquake (after Seed et al., 1976).

$$
\frac{\partial S}{\partial t}+n \rho_{\mathrm{f}} g \nabla z-n \rho_{\mathrm{f}} \frac{\partial v}{\partial t}-n^{2} \rho_{\mathrm{f}} \frac{g}{K} V_{\mathrm{r}}=n \rho_{\mathrm{f}} \frac{\partial V_{\mathrm{r}}}{\partial t}
$$

mass balance equation of pore water

$$
\frac{\partial S}{\partial t}=\frac{1}{C_{\mathrm{w}}} \frac{\partial v}{\partial z}+\frac{1}{C_{\mathrm{w}}} \frac{\partial V_{\mathrm{r}}}{\partial z}
$$

and time derivative of the stress-strain relation

$$
\frac{\partial S}{\partial t}=\left(\frac{1}{C_{\mathrm{c}}}+\frac{1}{n C_{\mathrm{w}}}\right) \frac{\partial V_{\mathrm{r}}}{\partial z}+\frac{1}{C_{\mathrm{w}}} \frac{\partial V_{\mathrm{r}}}{\partial z}-\frac{1}{C_{\mathrm{c}}}\left(\sigma-\frac{S}{n}\right) \frac{\partial C_{\mathrm{c}}}{\partial t}
$$

where

$$
S=-n p, \quad \sigma=-\sigma^{\prime}+\frac{S}{n}, \quad V_{\mathrm{r}}=\frac{\partial(U-u)}{\partial t}
$$


$C_{\mathrm{w}}$ is the compressibility of water, and $\left(1 / C_{\mathrm{c}}\right)$ is the secant modulus of the soil skeleton. These four equations form a hyperbolic system to be solved by the method of characteristics. The first two equations (equations (4.4) and (4.5)) are similar to equations (2.48) and (2.49). The coupled solutions of shear wave and pressure wave propagation have been presented by Liou et al. to simulate Niigate earthquake.

Endochronic modeling of two phase porous medium was developed by Bazant and Krizek (1975, 1976) after the work of Valanis (1971). Bazant and Krizek combined the endochronic constitutive equations with governing equations to analyze the liquefaction phenomenon. Bazant et al. (1982) and Valanis and Read (1982) reviewed endochronic models for soils. This theory which is different from the conventional stress-strain relations are separated into a relation for the volumetric components, and another one for the deviatoric components. Inelastic behavior which is produced by the deviatoric strain increments is described by the endochronic law. A similar approach was also employed by Sawicki and Morland (1985) for dry and saturated sand by adding elastic and non-linear irreversible deformations.

Hiremath and Sandhu (1984) and Morland et al. (1987) applied their numerical solution techniques to study liquefaction problems. They noted that, in general, for long wave-length problems with strong coupling like liquefaction, the relative motion of fluid and solid which maximized the pore pressure has been neglected. Sandhu and his coworkers' numerical solution are discussed in Section 3.2

Ghaboussi and Dikmen (1978) treated horizontal layers of saturated sand as fluid saturated porous media in their analysis of seismic response and evaluation of liquefaction potential. Coupled conservation of momentum equations were solved with nonlinear soil properties such as yield, failure, and cyclic effects. Later, Ghaboussi and Dikmen (1981) extended their analysis to three dimensional earthquake base acceleration. Zienkiewicz et al. $(1978,1982)$ presented a numerical solution with non-associative plasticity models. A review of these works are given by Zienkiewicz (1982). A similar approach with an elastoplastic solid matrix was also taken by Vardoulakis (1987).

\section{Wave propagation in unsaturated porous media}

In contrast to saturated porous media, wave propagation in unsaturated porous media received little attention from researchers. The general trend is to extend the Biot formulation developed for saturated medium to unsaturated medium by replacing model parameters with the ones modified for air-water mixture. Modification is generally done by volume averaging the density and the compressibility coefficients. For example, Spooner's (1971) equation of motion [equation (6.1)] contained a correction term to incorporate the degree of saturation in the inertia term. As an alternative, as noted in Section 7.1, others increased the volume compressibility of water due to trapped air in the porous medium (e.g., van der Grinten et al. 1987b). In addition to Verruijt's (1969) formula, we might 
also note Bishop and Eldin's (1950) expression for the compressibility of pore airwater mixture, $C_{w}$, as given by Ghaboussi and Kim (1984)

$$
C_{\mathrm{w}}=\left(1-S_{\mathrm{wo}}-H_{\mathrm{c}} S_{\mathrm{wo}}\right) \frac{p_{\mathrm{ao}}}{p^{2}}
$$

where $S_{\mathrm{wo}}$ is the initial degree of saturation. $H_{\mathrm{c}}$ is the solubility coefficient, $p_{\mathrm{ao}}$ is the initial pore air pressure, and $p$ is the pore water pressure. Schurman (1966) considered the surface tension between the air and the water $\left(=0.5\left(p_{\mathrm{a}}-p\right) R_{\mathrm{a}}\right)$ which is neglected in equation (5.1a). $R_{\mathrm{a}}$ is the radius of the air bubble, and $p_{\mathrm{a}}$ is the air pressure. Domenico (1974) defined the effective compressibility of the fluid, $\beta$, as

$$
\beta=S_{\mathrm{w}} \beta_{\mathrm{g}}+S_{\mathrm{w}} \beta_{\mathrm{w}}
$$

where $\beta_{\mathrm{g}}$ and $\beta_{\mathrm{w}}$ are the compressibility coefficient of the gas and the water, respectively. Composite density $\rho$ is obtained by adding equations (5.3) through (5.5). A similar approach was taken by Mochizuki (1982) by mass averaged parameters. Bedford and Stern (1983) developed a mixture theory for porous media saturated with a bubbly liquid which is equivalent to the Biot theory except that the inertial effect of bubble oscillations is included.

Brandt (1960) reported that in a water saturated quartz sand column, compressional wave velocity decreases linearly with the decreasing degree of water saturation, and levels off at 50\% saturation. Gassman (1951) employed the "distinct element" technique by representing the medium by packed elastic spheres (see Section 1). Brutsaert (1964) employed Lagrangian formulation similar to Biot's (1956a) approach to obtain a mathematical model. By taking $\rho_{12}=0$, the kinetic energy, $T$ of an unsaturated porous medium was expressed by

$$
\begin{aligned}
2 T= & \rho_{11}^{\prime}\left[\left(\frac{\partial u_{x}}{\partial t}\right)^{2}+\left(\frac{\partial u_{y}}{\partial t}\right)^{2}+\left(\frac{\partial u_{z}}{\partial t}\right)^{2}\right]+\rho_{22}^{\prime}\left[\left(\frac{\partial u_{x}^{\mathrm{g}}}{\partial t}\right)^{2}+\left(\frac{\partial u_{y}^{\mathrm{g}}}{\partial t}\right)^{2}+\left(\frac{\partial u_{z}^{\mathrm{g}}}{\partial t}\right)^{2}\right] \\
& +\rho_{33}^{\prime}\left[\left(\frac{\partial U_{x}}{\partial t}\right)^{2}+\left(\frac{\partial U_{y}}{\partial t}\right)^{2}+\left(\frac{\partial U_{z}}{\partial t}\right)^{2}\right]
\end{aligned}
$$

where $u^{\mathrm{g}}$ is the gas displacement. Mass coefficients $\rho_{11}^{\prime}, \rho_{22}^{\prime}, \rho_{33}^{\prime}$ are given by

$$
\begin{aligned}
& \rho_{11}^{\prime}=\rho_{11}=(1-n) \rho_{\mathrm{s}} \\
& \rho_{22}^{\prime}=\rho_{\mathrm{g}}\left(1-S_{\mathrm{w}}\right) n \\
& \rho_{33}^{\prime}=\rho_{\mathrm{f}} S_{\mathrm{w}} n
\end{aligned}
$$

where $\rho_{\mathrm{g}}$ is the density of gas. Equations (2.8) through (2.13) and (2.16) were generalized to include the dilatation of the gas, and an equation similar to equation (2.16) was proposed for the stress in the gas. The dissipation function given in equation (2.18) was modified to include the relative velocities between the gas and the solid, and the gas and the liquid phases. After this extension of Biot's theory, Brutsaert and Luthin (1964) provided experimental data which agrees with Brandt's (1960) conclusions. Also, Allen et al. (1980) provided laboratory data to 
evaluate the relationships between degree of saturation, pore pressure, time, and compression wave velocity.

Garg and Nayfeh (1986) developed a mixture theory by neglecting inertial coupling $\left(\rho_{12}=0\right)$. Momentum exchange between phases was incorporated by including the relative velocities between the gas and the solid, and the gas and the liquid phases in the momentum balance equations of respective phases. The coefficient $b$ was replaced by

$$
\begin{aligned}
& b_{\mathrm{sf}}=n^{2}\left(1-S_{\mathrm{w}}\right)^{2} \mu_{\mathrm{f}} /\left(k_{\mathrm{o}} k_{\mathrm{rw}}\right) \\
& b_{\mathrm{sg}}=n^{2} s_{w}^{2} \mu_{\mathrm{g}} /\left(k_{\mathrm{o}} k_{\mathrm{rg}}\right) \\
& b_{\mathrm{fg}}=0
\end{aligned}
$$

where $k_{\circ}$ is the intrinsic permeability of the medium, $k_{\mathrm{rw}}$ and $k_{\mathrm{ro}}$ are relative permeabilities for the water and the gas phase, respectively. $\mu_{\mathrm{f}}$ and $\mu_{\mathrm{g}}$ are respective viscosities. Equation (5.8) implies that there is no momentum transfer between two fluid phases due to negligible contact area between the water and the gas phase. Solubility of gas in water is incorporated in the model. Garg and Nayfeh's work is limited to low frequencies. At high frequencies $b_{\mathrm{sf}}, b_{\mathrm{sg}}$, and $b_{\mathrm{fg}}$ may not be constant, and furthermore, these constants and the capillary pressure $\left(p_{\mathrm{a}}-p_{\mathrm{w}}\right)$ should be taken as functions of frequency. Garg and Nayfeh assume linear elastic constitutive relations for all phases. Their solution for dilatational waves show three modes of propagation for weak viscous coupling. Three fronts merge into one with strong viscous coupling.

Kansa (1987, 1988, 1989) and Kansa et al. (1987) solved governing equations similar to that of Garg and Nayfeh (1986) by using an explicit Lagrangian code. They concluded that due to its small inertia, the gas phase response is basically uncoupled from solid and liquid phases. Gas phase also moves out of pores ("drained behavior") very readily in comparison to water which has a much larger inertia.

Based on their previous works (e.g., Berryman and Thigpen, 1985a,b,c,d), Berryman et al. (1988) presented a mixture theory for dilatational wave propagation. Their kinetic energy expression included terms for microstructural kinetic energy due to the dynamics of local expansion and contraction of individual phases and virtual mass due to relative flow of each phase in addition to usual kinetic energy terms given by equation (5.2). Drag coefficients were identical to that of Garg and Nayfeh (1986) (see equations (5.6) through (5.8)). However, Berryman et al. included the virtual mass effect in their formulation. They have shown that by neglecting effects due to changes in capillary pressure, governing equations reduce to equations similar to that of Biot for full saturation. Equation parameters incorporated the presence of the gas phase. This conclusion is analogous to the concept of replacing the coefficients of Biot equations with the ones modified for air-water mixture. Such an approach was reviewed earlier. Berryman et al.'s (1988) model can be expressed by

$$
\begin{aligned}
& \mu^{*} \nabla^{2} u+\left(H-\mu^{*}\right) \nabla \epsilon-c \nabla \xi+\omega^{2}\left(\rho_{\mathrm{uu}} u+\rho_{\mathrm{uw}} w\right)=0 \\
& C \nabla e-M \nabla \xi+\omega^{2}\left(\rho_{\mathrm{wu}} u+\rho_{\mathrm{ww}} w\right)=0
\end{aligned}
$$


where $\mu^{*}, H, C$, and $M$ are parameters similar to that of Biot's. However, the inertial coefficients $\rho_{\mathrm{uu}}, \rho_{\mathrm{uw}}$, and $\rho_{\mathrm{ww}}$, are much more complicated due to presence of gas phase. $\xi$ is the divergence of total fluid (water plus gas) displacements. In derivation of equations (5.9) and (5.10), Berryman et al. introduced a Fourier time dependence of the form $\exp (-i \omega t)(\omega=$ angular frequency) into the formulation. A comparison of equations (5.9) and (5.10) with equations (2.48) and (2.49) would demonstrate the analogy. Equation variables are identical in both sets of equations. Auriault et al. (1989) followed a similar approach by treating porous medium as a periodic media. Auriault et al. did not neglect the capillary pressures in their theoretical formulation. Lebaigue et al. (1987) applied this theory to analyze ultrasonic waves in a sheet of unsaturated wet paper. Ross et al. (1989) measured stress wave attenuation using the split Hopkinson pressure bar.

Santos et al. (1990b) presented a theory describing the wave propagation in a porous medium saturated by a mixture of two immiscible, viscous, compressible fluids by employing the principle of virtual complementary work. It was assumed that the two-phase flow in porous media obeys Darcy's law. Santos et al. (1990b) found that there are five possible body waves. Three of them correspond to compressional waves, and the other two, of identical speed, are associated with shear modes. This is a generalization of the single-phase Biot theory. The third kind dilatational wave is associated with the relative motion between two fluid phases. However, we must note that Darcy's law was not generalized to account for the relative motion of different phase fluids. The relative motion between the fluids might create a momentum exchange which in turn introduces additional head loss. Yuster (1951) tried to explain this by the remark that there is a shear transmitted at the two-phase interface which would actually entail such a phenomenon. A further discussion of the "Yuster effect" has been given by Scott and Rose (1953).

Santos et al. (1990b) stated the conservation of mass equation for oil and water phases as

$$
\begin{aligned}
& \frac{\partial\left(n \rho_{\mathrm{o}} S_{\mathrm{o}}\right)}{\partial t}+\nabla \cdot\left(\rho_{\mathrm{o}} K \frac{k_{\mathrm{ro}}}{\mu_{\mathrm{o}}} \nabla p_{\mathrm{o}}\right) \\
& \frac{\partial\left(n \rho_{\mathrm{w}} S_{\mathrm{w}}\right)}{\partial t}+\nabla \cdot\left(\rho_{\mathrm{w}} K \frac{k_{\mathrm{rw}}}{\mu_{\mathrm{w}}} \nabla p_{\mathrm{w}}\right)
\end{aligned}
$$

where $S_{\mathrm{o}}$ and $S_{\mathrm{w}}$ denote the oil and water saturations, respectively. Note that

$$
S_{\mathrm{o}}+S_{\mathrm{w}}=1
$$

Oil and water densities are denoted by $\rho_{\mathrm{o}}$ and $\rho_{\mathrm{w}}$, respectively. $K$ is the intrinsic permeability. $k_{\mathrm{ro}}$ and $k_{\mathrm{rw}}$ are the relative permeability functions for the oil and water, respectively. They are expressed in terms of $S_{\mathrm{w}} . \mu_{\mathrm{o}}$ and $\mu_{\mathrm{w}}$ denote the dynamic viscosities of the oil and water phases, respectively. $p_{\mathrm{o}}$ and $p_{\mathrm{w}}$ are the incremental oil and water pressures, respectively. Similar to Biot's work (see Section 2.2), the Lagrangian formulation of the equations of motion was stated by employing the kinetic energy density and dissipation energy density function 
definitions. Then, using the assumption of time independence for the saturation, a linearization technique, and the assumption of constant coefficients, Santos et al. (1990b) obtained the wave propagation equations

$$
\begin{aligned}
& \rho \frac{\partial^{2} u_{\mathrm{s}}}{\partial t^{2}}+\rho_{\mathrm{o}} \bar{S}_{\mathrm{o}} \frac{\partial^{2} u^{\mathrm{o}}}{\partial t^{2}}+\rho_{\mathrm{w}} \bar{S}_{\mathrm{w}} \frac{\partial^{2} u^{\mathrm{w}}}{\partial t^{2}}=N \nabla^{2} u^{\mathrm{s}}+\nabla\left[(\lambda+N) \epsilon+B_{1} e^{\mathrm{o}}+B_{2} e^{\mathrm{w}}\right] \\
& \rho_{\mathrm{o}} \bar{S}_{\mathrm{o}} \frac{\partial^{2} u^{\mathrm{s}}}{\partial t^{2}}+\bar{g}_{1} \frac{\partial^{2} u^{\mathrm{o}}}{\partial t^{2}}+\bar{g}_{3} \frac{\partial^{2} u^{\mathrm{w}}}{\partial t^{2}}+\left(\bar{S}_{\mathrm{o}}\right)^{2} \frac{\mu_{\mathrm{o}}}{K k_{\mathrm{ro}}} \frac{\partial u^{\mathrm{o}}}{\partial t}=\nabla\left[B_{1} \epsilon+M_{1} e^{\mathrm{o}}+M_{3} e^{\mathrm{w}}\right] \\
& \rho_{\mathrm{w}} \bar{S}_{\mathrm{w}} \frac{\partial^{2} u^{\mathrm{s}}}{\partial t^{2}}+\bar{g}_{3} \frac{\partial^{2} u^{\mathrm{o}}}{\partial t^{2}}+\bar{g}_{2} \frac{\partial^{2} u^{\mathrm{w}}}{\partial t^{2}}+\left(\bar{S}_{\mathrm{w}}\right)^{2} \frac{\mu_{\mathrm{w}}}{K k_{\mathrm{rw}}} \frac{\partial u^{\mathrm{w}}}{\partial t}=\nabla\left[B_{2} \epsilon+M_{3} e^{\mathrm{o}}+M_{2} e^{\mathrm{w}}\right]
\end{aligned}
$$

where $u^{\mathrm{s}}, u^{\mathrm{o}}$, and $u^{\mathrm{w}}$ denote solid, oil, and water phase displacements, respectively. An overbar refers to a reference value. $\epsilon, e^{\circ}$, and $e^{\mathrm{w}}$ denote volumetric dilatations of the solid, oil, and water phases respectively. $\rho$ is defined by

$$
\rho=(1-n) \rho_{\mathrm{s}}+n\left(\rho_{\mathrm{o}} S_{\mathrm{o}}+\rho_{\mathrm{w}} S_{\mathrm{w}}\right)
$$

where $\rho_{\mathrm{s}}$ is the solid density. $\lambda, N, g_{\mathrm{c}}, B_{1}, B_{2}, g_{1}, g_{2}, M_{1}, M_{2}$, and $M_{3}$ are all material parameters. In formulating equations (5.14)-(5.16), Santos et al. ignored the friction effects between the oil and water phases in the dissipation energy function. We must note that instead of using the Lagrangian formulation to obtain the wave propagation equation, we can state the momentum conservation equations as shown by Baer and Corapcioglu (1989) (see Section 9). Santos et al. (1990b) obtained the equations governing the propagation of dilatational and rotational waves by applying the divergence and curl operators to equations (5.14) $-(5.16)$, respectively.

In a companion paper, following the presentation of a method to determine the elastic constants for an isotropic porous medium saturated by a two-phase fluid, Santos et al. (1990a) calculated the phase velocities and attenuations for Berea sandstone saturated by mixtures of oil and water, and gas and water as functions of both frequency and saturation of the non-wetting phase. As shown in Figures 16, 17, and 19, Santos et al. observed that for low saturations, the phase velocities of first and second kind compressional waves, and shear waves approach to the corresponding water-saturated (i.e., $S_{\mathrm{w}}=1$ ) porous medium. As seen in Figure 17, the wave in gas-water mixture saturated medium is slower due to smaller relative motion of lower density fluids. The third kind dilatational wave which is directly associated with the presence of capillary pressures, increases with the saturation of non-wetting phase (oil or gas) (see Fig. 18. The phase velocity of the third kind wave is much slower than others and reaches to zero velocity at low saturations. Similar to the second kind waves, the third kind dilatational waves are diffusion-type waves. As seen in Fig. 19, the shear wave phase velocity increases almost linearly with the non-wetting phase saturation for both mixtures. Since, the bulk density of the gas-water mixture saturated porous medium is 


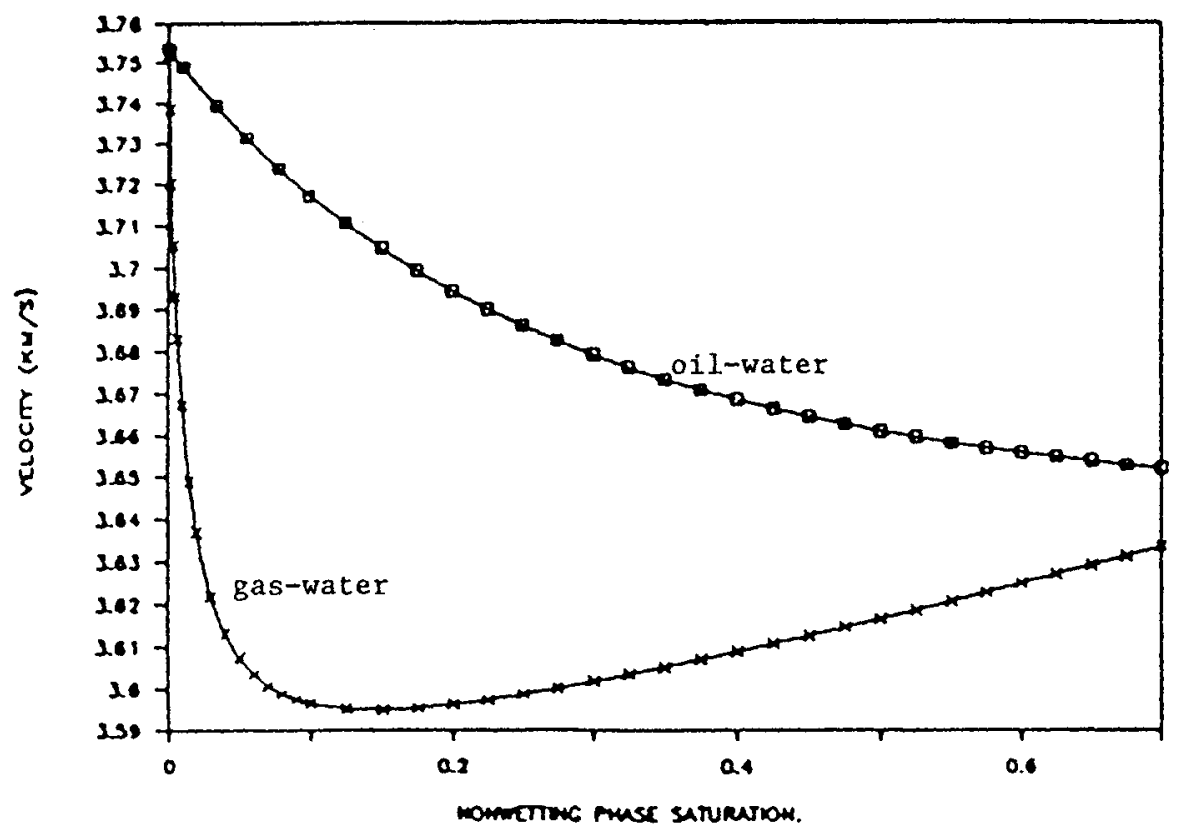

Fig. 16. Phase velocity of dilatational waves of the first kind (after Santos et al., 1990a).

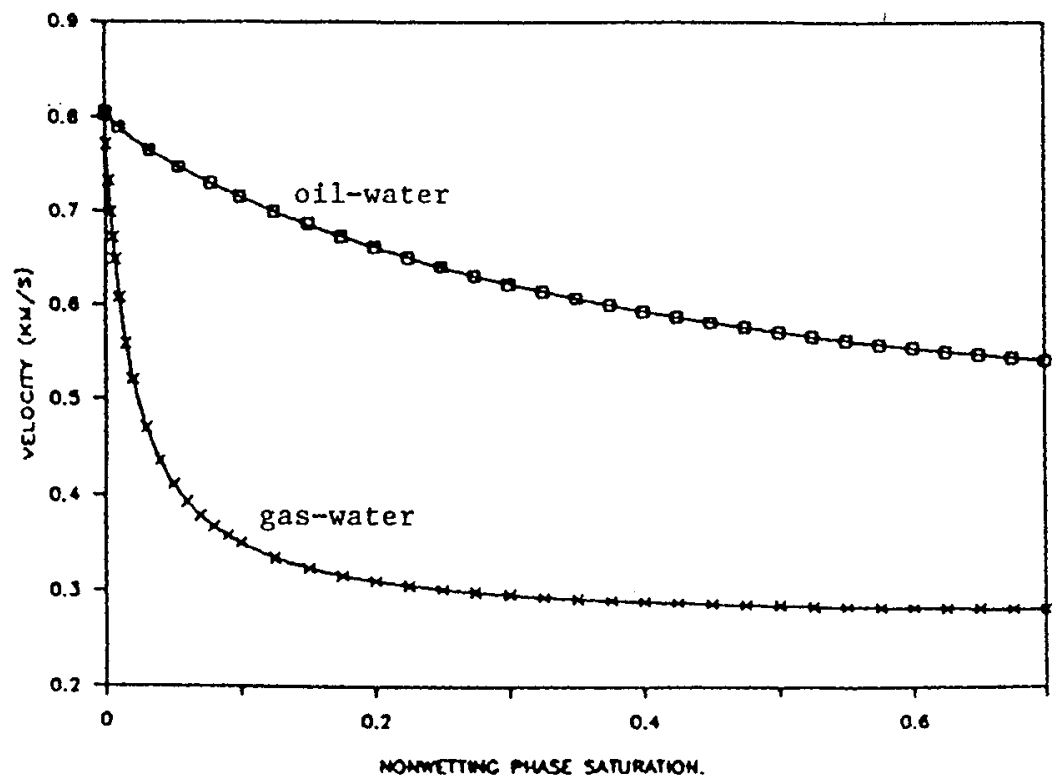

Fig. 17. Phase velocity of dilatational waves of the second kind (after Santos et al., 1990a). 


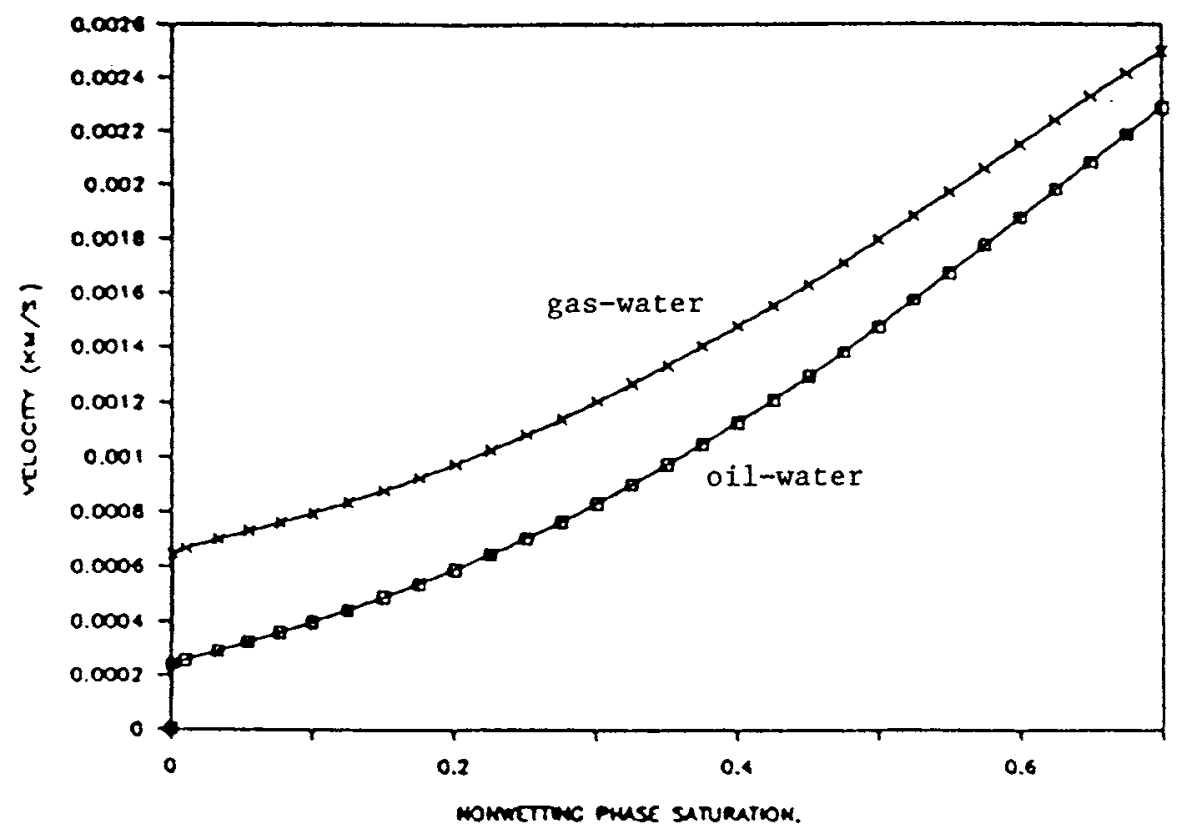

Fig. 18. Phase velocity of dilatational waves of the third kind (after Santos et al., 1990a).

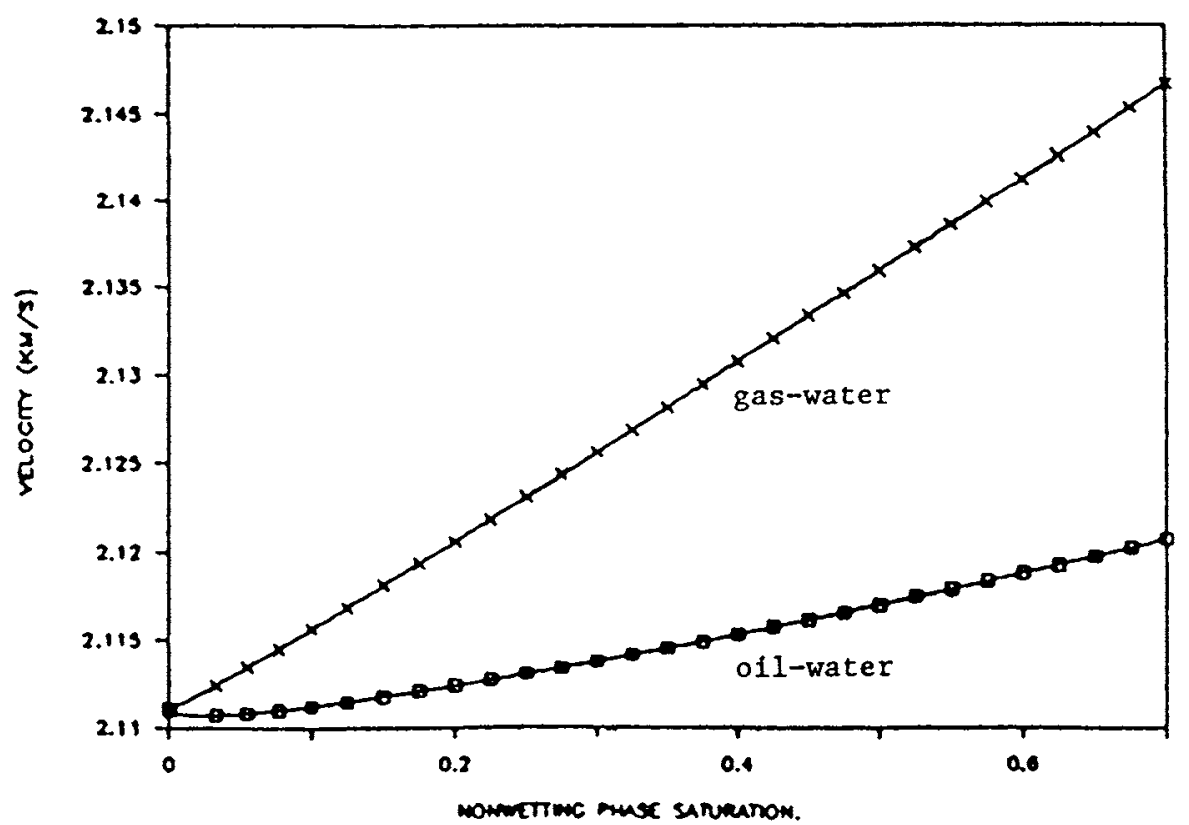

Fig. 19. Phase velocity of shear wave (after Santos et al., 1990a). 
smaller than the oil-water saturated medium, it has higher shear wave phase velocity. Santos et al.'s model shows increasing phase velocities with frequency at low frequencies $(<5 \mathrm{~Hz})$ and almost constant velocities at higher frequencies. They questioned the validity of their model at high frequencies, since the frequency dependence of dissipation for high frequencies has not been taken into account in their formulation (see Section 2.5). The attenuation coefficients of the first kind dilatational and shear waves were found to be almost zero at low frequencies and very small at high frequencies with peaks at a particular frequency.

White (1975) derived expressions by extending Gassmann's viewpoint to include coupling between the fluids. White considered a spherical region of gas or liquid surrounded by a concentric shell of liquid or gas. Norris (1992) developed a macroscopic theory that takes into account the type of microstructure in White's model.

Domenico $(1974,1976)$ experimentally investigated the effects of water saturation on reflection, refraction, and phase velocity of body waves and showed that the drainage process used in many laboratory studies (Gregory, 1976; Elliot and Wiley, 1975; Domenico, 1976) results in extremely heterogeneous gas distribution in the samples.

Murphy (1982) provided further data on the effects of partial water saturation on attenuation in high-porosity sandstone $(n=0.23)$ and porous glass $(n=0.28)$. Murphy concluded that attenuation is much more sensitive to degree of saturation than the wave velocity. In partially saturated sandstones, attenuation is strongly frequency dependent. Murphy also concluded that viscous dissipation is dominant over surface film mechanism at saturation levels over one percent. At very low saturations, monolayer of water react with the silicate surface, increasing the compressibility of the matrix significantly. Biot's theory does not take this effect into consideration. Murphy (1984) observed different results in tight sandstones $(n=0.033-0.085)$ which can be explained by contact relaxation mechanism. Although grain-to-grain response is elastic, water trapped in the contact gap adds a component stiffness to the matrix and creates a viscoelastic response.

Yin. et. al. (1992) used force-deformation method to examine the attenuation characteristics of unsaturated porous media and concluded that pore fluids within the rock affect attenuation not only by their degree of saturation but also by the history of the saturation.

\section{Use of wave propagation equation to estimate permeability}

An inspection of equation (2.19) reveals that the coefficient $b$ of the dissipation term is related to the intrinsic permeability of the medium. Klimentos and McCann (1990) found relationships between the clay content, phase velocity and attenuation. Their results showed that the relationship between clay content and attenuation is very strong. Since permeability strongly depends on the clay content, dissipation is the key factor in determining the permeability of a porous medium. We should note that permeability is measured by quasi-static experiments where there is net flow. However, in wave propagation there is no net flow but oscil- 


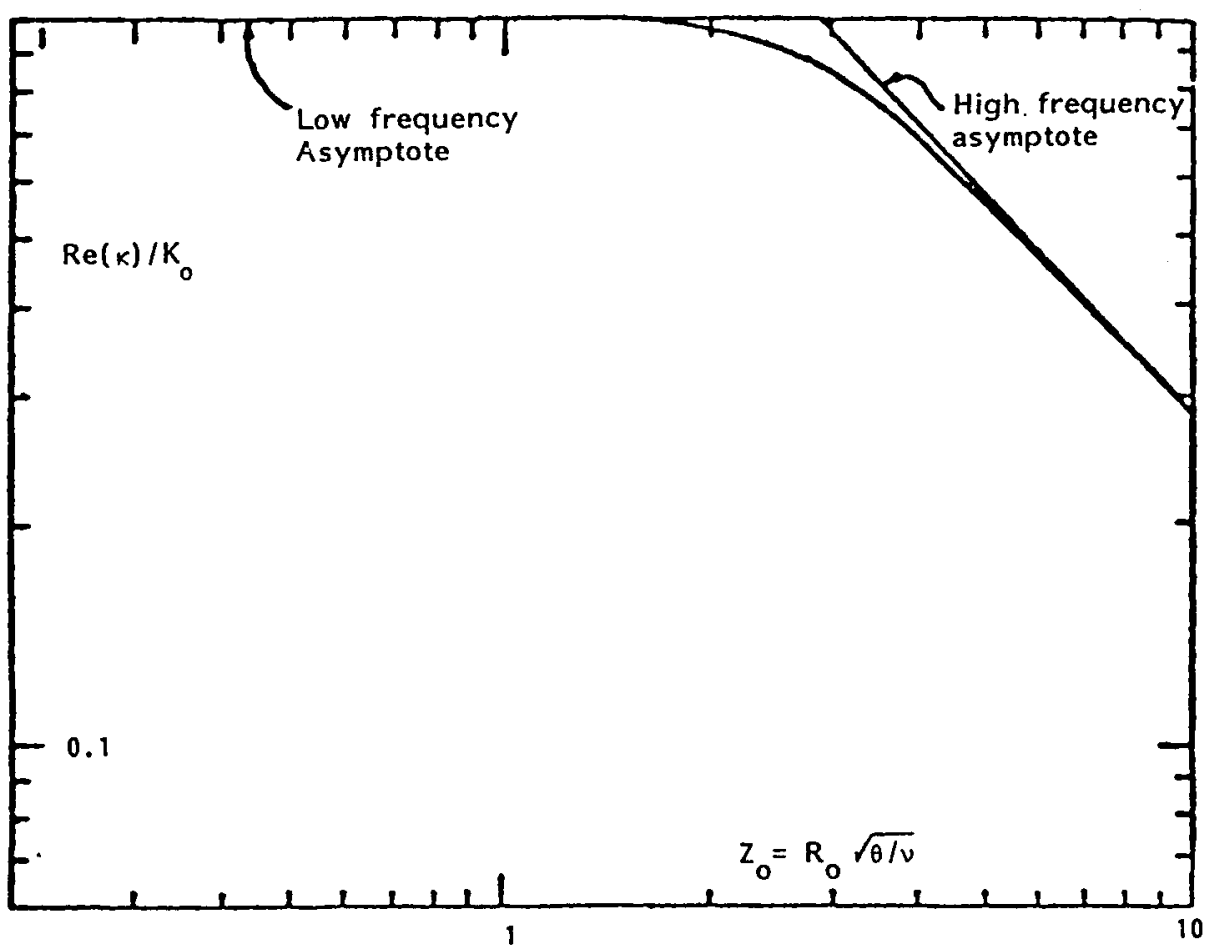

Fig. 20. Frequency dependence of hydraulic conductivity (after Misra and Monkmeyer, 1966).

lations about an equilibrium position. Hence, permeability that is back calculated by using wave propagation theory may differ from the quasi-static permeability of a porous medium. Similar statements were made by Berryman (1986b, 1988). Berryman warned against the use of Biot's theory to determine the permeability of rocks in the low-frequency range $(1-100 \mathrm{~Hz})$. He showed that since the intrinsic permeability of the rock is inhomogeneous and varies widely in magnitude, the spatial scale of Biot's theory is quite small. Therefore, Biot's theory predicts an order of magnitude of different permeability values than measured permeabilities. Nagy (1993) concluded that the observed discrepancy in attenuation coefficients is due to the irregular geometry that significantly reduces the high frequency dynamic permeability. Mochizuki (1982) argued that attenuation measurements of Murphy (1982) can not be explained by Biot's theory. Prasad and Meissner (1992) observed that other attenuation mechanisms exist in saturated sands. The discrepancy between Biot's theory and experimental results are believed to be because of the squirt flow in the microscale (Mavko and Nur, 1979; Murphy et al., 1986; Akbar et al., 1986). Some researchers used wave propagation equations to estimate the permeability of the medium. In this section, we will review some of these studies.

Wylie et al. (1962) suggested the use of Biot's theory to calculate the hydraulic conductivity by measuring the attenuation at two or more frequencies. However, they did not introduce a formalism to their proposal. 
Spooner (1971) obtained the wave propagation equation for a partially saturated porous medium in terms of pore pressure by taking the divergence of the conservation of momentum equation

$$
\frac{\left(S_{\mathrm{w}} \rho_{\mathrm{f}}+\left(1-S_{\mathrm{w}} \rho_{\mathrm{g}}\right)\right) m}{n} \frac{\partial q_{\mathrm{r}}}{\partial t}=-\nabla p-\frac{\mu_{\mathrm{f}}}{k} q_{\mathrm{r}}
$$

and differentiating the conservation of mass equation for the water phase

$$
-\nabla \cdot \boldsymbol{q}_{\mathrm{r}}=\left(S_{\mathrm{w}} n \beta^{\prime \prime}+\frac{\rho_{\mathrm{g}}}{\rho_{\mathrm{f}}} \frac{\left(1-S_{\mathrm{w}}\right) n}{p_{0}}+\left[S_{\mathrm{w}}+\frac{\rho_{\mathrm{g}}}{\rho_{\mathrm{f}}}\left(1-S_{\mathrm{w}}\right)\right] a_{\mathrm{v}}\right) \frac{\partial p}{\partial t}
$$

and combining equations (6.1) and (6.2), and eliminating $\boldsymbol{q}_{\mathrm{r}}$

$$
\nabla^{2} p=m\left[S_{\mathrm{f}} \rho_{\mathrm{f}}+\left(1-S_{\mathrm{w}}\right) \rho_{\mathrm{g}}\right] \beta^{\prime} \frac{\partial^{2} p}{\partial t^{2}}+\frac{\mu_{\mathrm{f}} n}{k} \beta^{\prime} \frac{\partial p}{\partial t}
$$

where $m$ is the "structure factor" (see equation (2.52)) which is called mass coefficient by Biot. $S_{\mathrm{w}}$ is the degree of water saturation, $\rho_{\mathrm{g}}$ is the density of gas, $p_{\mathrm{o}}$ is the reference pressure. $a_{\mathrm{v}}$ is the coefficient of volume compressibility of the solid matrix $\left(=(1-n)^{-1} d n / d p\right) . \beta^{\prime \prime}$ is the compressibility of water. $\beta^{\prime}$ is considered as an "effective compressibility" and is equal to the coefficient on the right side of equation (6.2) divided by $n . \boldsymbol{q}_{\mathrm{r}}$ is the relative specific discharge of water

$$
\boldsymbol{q}_{\mathrm{r}}=n \frac{\partial}{\partial t}(\boldsymbol{U}-\boldsymbol{u})
$$

Note that $q_{\mathrm{r}}=\boldsymbol{w}$ [see equation (2.47)]. A comparison of equation (6.1) for $S_{\mathrm{w}}=$ 1 (i.e., saturated porous medium) with equation $(2.49)$ reveals that

$$
\begin{aligned}
& -n \nabla p=-\rho_{\mathrm{f}} m \frac{\partial^{2}}{\partial t^{2}}(u-U)-\frac{n^{2} \mu_{\mathrm{f}}}{k} \frac{\partial}{\partial t}(u-U) \\
& \nabla p=\frac{\partial^{2}}{\partial t^{2}}\left[\rho_{\mathrm{f}} u+m n(U-u)\right]+\frac{\mu_{\mathrm{f}}}{k} \frac{\partial}{\partial t}(U-u)
\end{aligned}
$$

The parameter $m$ has been introduced by Zwikker and Kosten (1949). It incorporates the increasing effect of "apparent density" $\left[S \rho_{\mathrm{f}}+(1-S) \rho_{\mathrm{g}}\right]$ in the inertial term of the fluid. Zwikker and Kosten comment that as seen in equation (6.5) the fluid flow may not be in the direction of pressure gradient due to increase in the apparent inertia of the fluid which results from the vibration of the solid matrix. Furthermore, Zwikker and Kosten showed that the "resistance constant" $(=1 / k)$ depends on the frequency of oscillation of the fluid. Zwikker and Kosten considered only two dilatational waves by using the concept of impedance. Same concept was also emphasized by Beranek (1947). The problem was also studied by Morse (1952) under the simplifying assumption of "rigid-frame theory." Rigidframe theory assumes that the pore fluid is air, and the solid matrix is considered rigid. With a rigid matrix, there is only one dilatational wave, and it travels through the air. Morse considers high frequency range when inertial effects dominate over 
viscous ones. He finds that $2.0<m<3.4 . m=2$ corresponds to uniform grain size materials while $m=3.4$ is for non-uniform granular porous media.

If we rewrite equation (6.3) in one-dimension

$$
\frac{\partial^{2} p}{\partial x^{2}}=\frac{m}{C_{o}^{2}} \frac{\partial^{2} p}{\partial t^{2}}+\frac{n \mu_{\mathrm{f}}}{\left[\rho_{\mathrm{f}} S_{\mathrm{w}}+\left(1-S_{\mathrm{w}}\right) \rho_{\mathrm{g}}\right] k C_{o}^{2}} \frac{\partial p}{\partial t}
$$

The wave velocity, $V_{\mathrm{c}}$, is given by

$$
V_{\mathrm{c}}=\frac{C_{\mathrm{o}}}{\sqrt{m}}=\left[\frac{1}{\left[\rho_{\mathrm{f}} S_{\mathrm{w}}+\left(1-S_{\mathrm{w}}\right) \rho_{\mathrm{g}}\right] m \beta^{\prime \prime}}\right]^{1 / 2}
$$

Misra (1965) and Misra and Monkmeyer (1966) assumed a plane, progressive harmonic wave solution for the fluid pressure as

$$
p(x, t)=p_{\mathrm{o}} e^{i(\theta t-\beta x)}
$$

where $r$ is the frequency, and $\beta$ is the complex wave number. The imaginary part of $\beta$ is the attenuation constant and the real part is the phase constant. Misra and Monkmeyer (1966) have shown that by using capillary tube modeling of the porous medium, the steady state hydraulic conductivity, $K_{\mathrm{o}}\left(=k \rho_{\mathrm{f}} g / \mu_{\mathrm{f}}\right)$ is given by

$$
K_{\mathrm{o}}=\frac{\rho_{\mathrm{f}} g n R_{o}^{2}}{8 \mu_{\mathrm{f}} m}
$$

where $R_{\mathrm{o}}$ is the radius of the capillary tube. During wave propagation, $K_{\mathrm{o}}$ is a function of $z_{\mathrm{o}}=R_{\mathrm{o}}\left(r^{*} / v_{\mathrm{f}}\right)^{1 / 2}$ where $v_{\mathrm{f}}$ is the kinematic viscosity. Misra and Monkmeyer have shown that for low frequencies, i.e., small $z_{\mathrm{o}}$ (or low permeabilities) the hydraulic conductivity approaches its static value (i.e., $K=K_{\mathrm{o}}$ ). For high frequencies (or high permeabilities), the permeability is proportional to the structure factor, $w$.

Geertsma (1974) defined the Reynolds number in terms of the "coefficient of inertial flow resistance"

$$
\operatorname{Re}=\frac{\chi \rho_{\mathrm{f}} q k}{\mu_{\mathrm{f}}}
$$

from the Forcheimer equation

$$
-\nabla p=\frac{\mu_{\mathrm{f}}}{k} q+\chi \rho_{\mathrm{g}}|q| \cdot q
$$

where $\boldsymbol{q}$ is the specific discharge of fluid (i.e., $\boldsymbol{q}=n \partial u / \partial t$ ) and $\chi$ is the coefficient of inertial flow resistance. A comparison with Biot equations show that $\chi$ is similar to $\rho_{12}$ (or $m$ ). The Reynolds number as given by equation (6.10) describes the upper limit of Darcy's law.

Smith and Greenkorn (1972) independently derived equation (6.3) and equation (6.9) for a saturated rigid porous medium, i.e., $a_{\mathrm{v}}=0, S=1$. Smith et al. (1974a) presented experimental data obtained in nitrogen filled Ottawa sand to check the validity of their theory. The results of Spooner (1971) and Smith and Greenhorn 
(1972a) are quite similar. Smith et al. (1972b) extended the theory to transient pressure response. Their results show that inertial effects exist for short distances and high permeabilities.

Turgut and Yamamoto (1990) studied attenuation of acoustic waves in fluid saturated sediments and obtained good agreement between the experimental and theoretical results. This enabled the remote estimation of porosity and permeability of marine sediments by using measured compressional and shear wave characteristics. They were able to estimate the porosity distribution of a $3 \times 3 \mathrm{~m}$ vertical plane by a cross-hole tomography experiment. Porosities are calculated from the compressional wave velocities which are inverted from measured travel times by using singular decomposition technique.

\section{Wave propagation in marine environments}

\subsection{Response of porous beds to water waves}

When sea waves propagate over a porous bottom, they induce fluid flow in the medium and cause the bed to deform. In shallow waters, fluctuating wave pressures can generate high levels of energy resulting in soil failure and damage to structures such as pipelines and offshore terminals. Therefore, numerous investigations were carried out with various degrees of simplifications. Assumptions of a rigid bed and incompressible water leads to the Laplace equation in terms of pore pressure $\left[\nabla^{2} p=0\right]$ (pressure waves), (e.g., Putnam, 1949; Reid and Kajiura, 1957; Oroveanu and Pascal, 1959; Sleath, 1970; Demars, 1983). Later, Moshagen and Torum (1975) introduced the compressibility of water, thus obtaining a diffusion type (parabolic) equation for the pore pressure. In contrast to Laplace equation, pore pressure response is highly affected by the permeability of the sea bed. Verruijt (1982) considered only standing waves in his analytical solution. Madsen (1978), Yamamoto et al. (1978), and Nataraja and Gill (1983) took into consideration the flow in the bed, compressibility of water and elastic bed in their formulation. Yamamoto et al. noted that even a very small amount of air trapped in the bed would increase the volume compressibility of water very drastically (Verruijt, 1969).

$$
\beta=\beta_{\mathrm{o}}+\frac{\left(1-S_{\mathrm{w}}\right)}{p_{\mathrm{o}}}
$$

where $\beta$ and $\beta_{\mathrm{o}}$ are the compressibility coefficient of water and pure water, respectively. $S_{\mathrm{w}}$ is the degree of water saturation, $p_{\mathrm{o}}$ is the absolute pore pressure (taken as $1 \mathrm{~atm}$ by Yamamoto et al., 1978).

Yamamoto and Takahashi (1983) established a Froude-Mach similitude law for sea-seabed interaction. This law requires that three Mach numbers which are the ratios of water wave phase velocity to the velocities of the fast and slow compressional waves and the shear waves in the seabed should be equal in the prototype and physical scale model in addition to the geometric similarity and the 
Froude number squared which is the ratio of inertial to gravity force. In general, the Mach number is a ratio of inertial to elastic force and it is an indicator of the importance of compressibility effects in a fluid flow. When the Mach number is small, the associated inertial force does not cause significant compressibility. Yamamoto and Takahashi found that the response of sand beds to water waves is linear and quasi-static. However, clay beds showed highly nonlinear and dynamically amplified response. This conclusion enforces the concept of internal loss due to the Coulomb friction between clay particles which is independent of loading frequency (Yamamoto and Schuckman, 1984). Therefore, the representation of seabed as a "fluid-like" material (e.g., Dalrymple and Liu, 1978) is inadequate.

In all these studies, the inertia term in the momentum balance equations were neglected. Although Massel (1976) included the inertia term in his equation, he concluded that the effect of permeability on the pressure variation is negligible, thus the governing equation gives results similar to that of the Laplace equation. Dalrymple and Lui (1982) extended Yamamoto's work to include the inertia term in the governing equations. They concluded that the inertial terms are important when a dimensionless parameter which is the ratio of the square of the wave speed for an elastic solid to the water wave speed, is close to one. When this parameter is less than one (i.e., soft sediments), the solid displacements and the shear stress, $\tau_{x y}$, oscillate as they decay with depth. They further noted that $\rho_{12}$ has negligible effects on solutions. Later, Liu and Dalrymple (1984) employed the generalized Darcy's law with an acceleration term obtained by Dagan (1979), to describe the oscillatory flow in soil bed. Basak and Madhav (1978), Wylie (1976), Wiggert and Wylie (1976), and Auriault et al. (1985) also included the acceleration term in Darcy's law. The inclusion of inertial effects can also be achieved by using the Forchheimer equation (Finjord, 1990).

Finn et al. (1982) reviewed the methods for estimating the response of seafloor to ocean waves and the determination of wave-induced pore pressures. Finn et al. have shown that transient pore pressures and the associated effective stress field may be investigated by Biot's (1941) theory of consolidation. We must note that by using this approach Finn et al. (1982) assume quasi-static (equilibrium) distribution of stresses.

As noted in Section 4, Seed and his coworkers (e.g., Seed and Rahman, 1978) introduced the concept of pore pressure generation under cyclic loading condition to investigate the response of seafloor sands subjected to storm wave loadings (see equation 4.1). Siddharthan (1987) combined this approach with that of Yamamoto and Madsen, to analyze the seafloor response to a storm wave group. Siddhartham found that for North Sea seafloor, the inclusion of inertia, damping and anisotropic permeabilities is not important. However, the thickness and the stiffness properties of the sediment govern the response of the deposit. Thus, the seafloor displacements are affected by residual pore pressures generated by waves.

\subsection{Mei and Foda's boundary layer theory}

Mei and Foda (1981) obtained a solution for Biot's equations for rapid water waves with high frequencies (i.e., ocean waves or seismic waves). Mei and Foda 


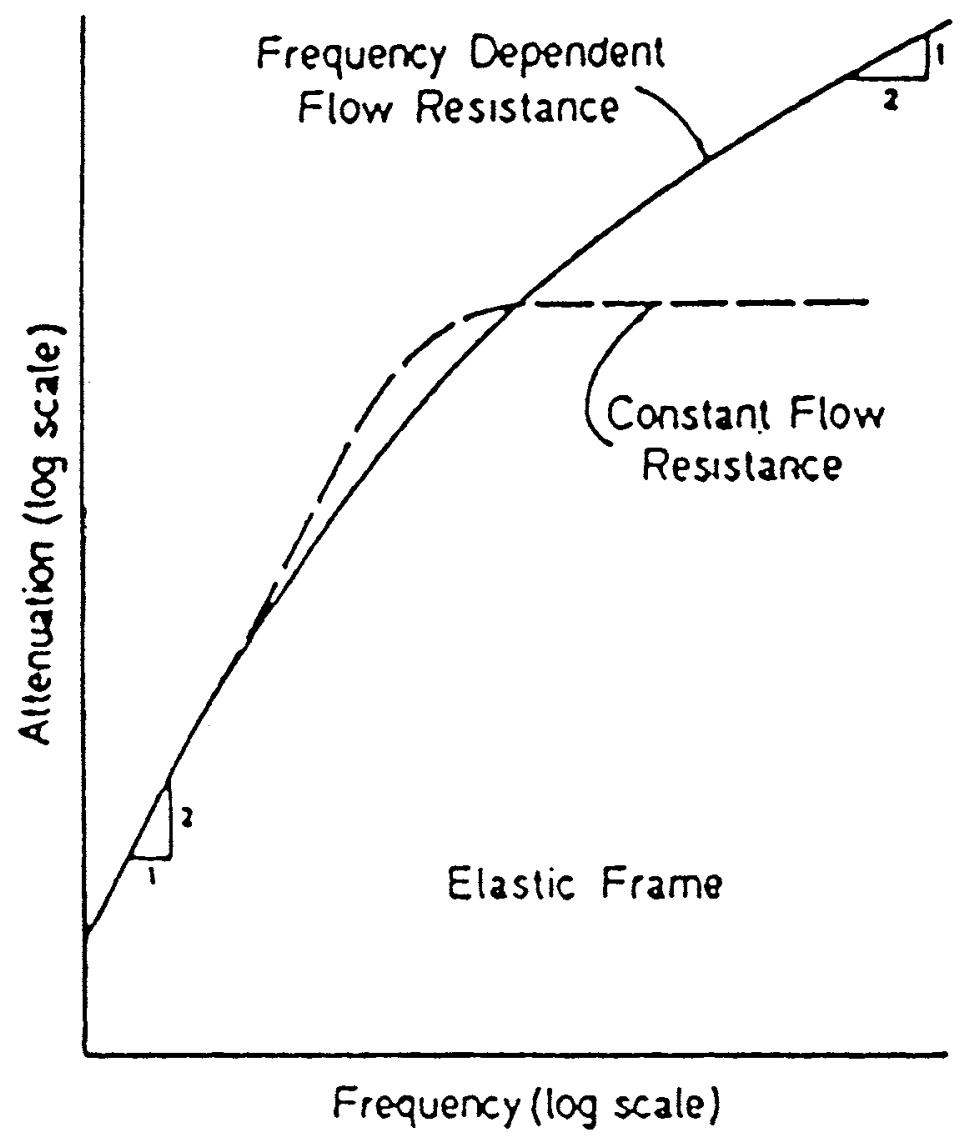

Fig. 21. Attentuation versus frequency for a linear elastic frame (after Stoll, 1974).

has concluded that the region close to the porous medium surface is drained and pore pressures in that region are independent of the wave length. The depth of this zone of consolidation is smaller than the wave length. This region is treated as a boundary layer of Stokes' type with one-dimensional flow. The boundary layer concept agrees well with the Biot's conclusion for the waves of the second kind which have very short attenuation distances and the disturbance propagates in a form similar to diffusion. Outside the boundary layer, the porous medium reacts undrained and the fluid and the solid matrix move together. Mei and Foda $(1981,1982)$ have shown that by neglecting the grain compressibility (unjacketed compressibility) and the apparent mass ( $\left.\rho_{12}\right)$ from equation (2.28) and using elastic strain relation [equation (2.16)], they obtain

$$
n \rho_{\mathrm{f}} \frac{\partial V_{x}}{\partial t}=-n \frac{\partial p}{\partial x}-\frac{n^{2}}{k^{*}}\left(V_{x}-v_{x}\right)
$$

$V_{x}$ and $v_{x}$ are the fluid and the solid velocities, respectively. Note that $v_{x}=$ $\partial u_{x} / \partial t$ and $V_{x}=\partial U_{x} / \partial t$. By neglecting some parameters and eliminating $\nabla . u$ from 


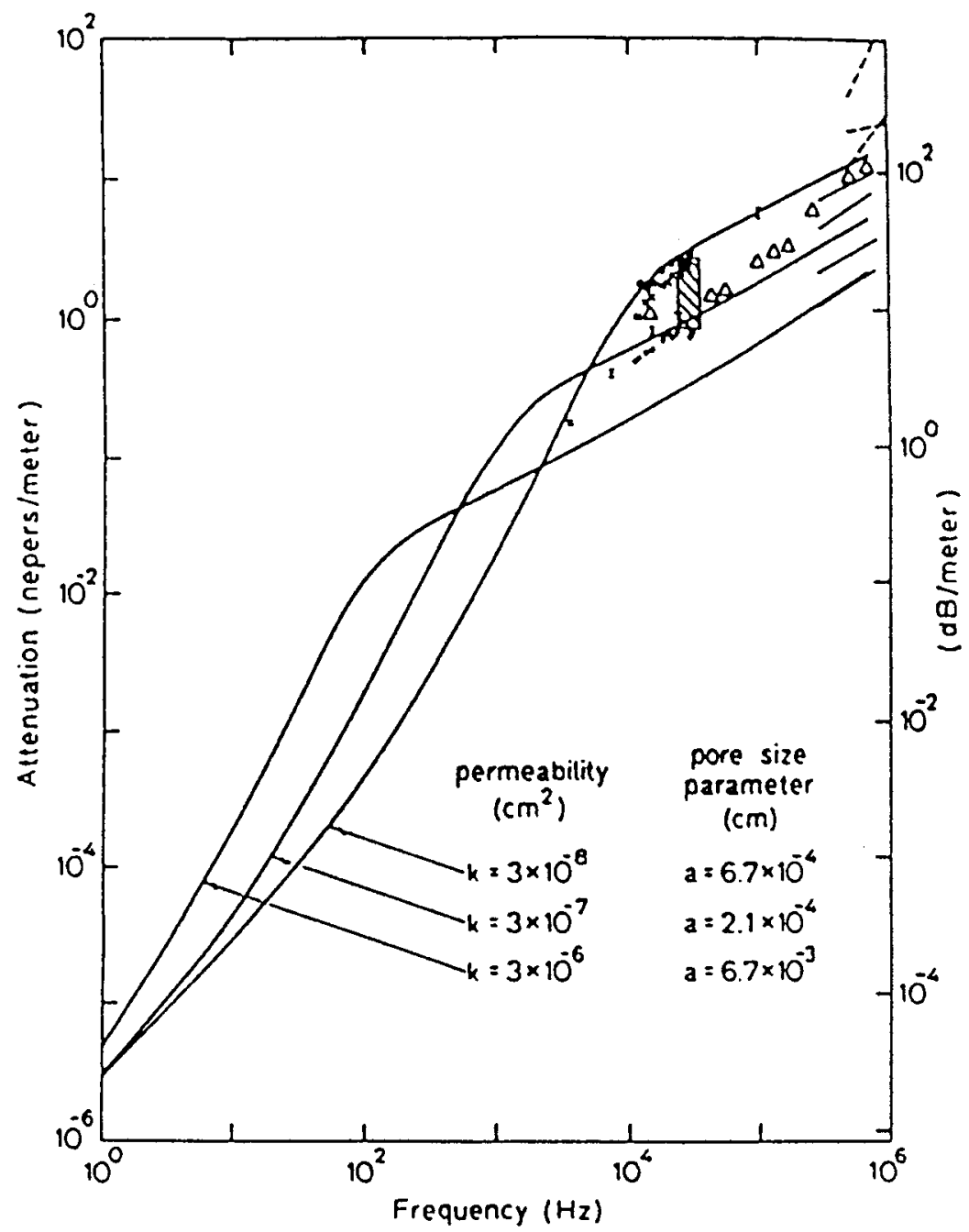

Fig. 22. Attenuation versus frequency for sands (after Stoll, 1974).

equation (2.25) by using equations (2.15) and (2.16) and inserting elastic stressstrain relations, Mei and Foda obtained,

$$
(1-n) \rho_{\mathrm{s}} \frac{\partial v_{x}}{\partial t}=\frac{\partial \sigma_{x x}}{\partial x}+\frac{\partial \tau_{x y}}{\partial y}+\frac{\partial \tau_{x z}}{\partial z}-(1-n) \frac{\partial p}{\partial x}+\frac{n^{2}}{k^{*}}\left(V_{x}-v_{x}\right)
$$

Note that Mei and Foda's $k^{*}$ is equal to Biot's $k / \mu_{\mathrm{f}}$. Similar equations can be obtained in the $y$ - and $z$-directions. The conservation of mass equations for the solid and the fluid phases

$$
\nabla \cdot\left[(1-n) \rho_{\mathrm{s}} v\right]+\frac{\partial\left[(1-n) \rho_{\mathrm{s}}\right]}{\partial t}=0
$$




$$
\nabla \cdot\left(n \rho_{\mathrm{f}} V\right)+\frac{\partial\left(n \rho_{\mathrm{f}}\right)}{\partial t}=0
$$

are combined to obtain (in $x$-direction)

$$
n \nabla \cdot(V-v)+\nabla \cdot v+\frac{n}{\beta} \frac{\partial p}{\partial t}=0
$$

where $\beta$ is the compressibility of water. Equation (7.5) has been obtained by various other researchers for the compressible groundwater aquifer problem (e.g., Bear and Corapcioglu, 1981).

By adding equations (7.1) and (7.2), and using the Hooke's law, Mei and Foda obtained

$$
G\left(\nabla^{2} v+\frac{1}{1-2 v} \nabla \nabla \cdot v\right)-\nabla \frac{\partial p}{\partial t}=n \rho_{\mathrm{f}} \frac{\partial^{2} V}{\partial t^{2}}+(1-n) \rho_{\mathrm{s}} \frac{\partial^{2} v}{\partial t^{2}}
$$

They eliminated $(\mathbf{V}-\mathbf{v})$ from equations (7.2) and (7.5)

$$
k^{*} \nabla^{2} p=\nabla \cdot v+\frac{n}{\beta} \frac{\partial p}{\partial t}-k^{*} \rho_{\mathrm{f}} \frac{\partial}{\partial t}(\nabla \cdot V)
$$

where $G$ and $\nu$ are the shear modulus and Poisson ratio, respectively. Mei and Foda split the stress field into the outer solution and the boundary layer correction. For outer solution $V=v$ and the first and the last term of equation (7.7) is neglected since, the dimensionless parameter of $\rho_{\mathrm{f}} \omega^{2} L^{2} / G$ is very small for the seismic wave length $L=100-500 \mathrm{~m}$, seismic frequency $\omega=10 \mathrm{rad} \mathrm{s}^{-1}$ and $\omega L^{2} / G k^{*}$ is very large. These parameters appear in equation (7.7) after a nondimensionalization is performed. The first one is the ratio of inertia to pressure (or stress) forces. The second is the ratio of Darcy's drag force to the pressure (or stress) gradient waves. Therefore the solid dilatation $\nabla . v$ is directly related to the pore pressure change. Then, the velocity of compressional waves is

$$
V_{\mathrm{c}}^{2}=\frac{\lambda_{\mathrm{e}}+2 G}{n \rho_{\mathrm{f}}+(1-n) \rho_{\mathrm{s}}}
$$

$\lambda_{\mathrm{e}}$ is the effective Lame constant $=\lambda+\beta / n$ where $\beta^{-1}$ is the compressibility of water. Shear waves propagate with a velocity of $V_{\mathrm{s} 2}=G /\left[\rho_{\mathrm{f}} n+(1-n) \rho_{\mathrm{s}}\right]$. For gravity waves at the sea with $\omega=0.5-1 \mathrm{rad} \mathrm{s}^{-1}, L=50-200 \mathrm{~m}$ same approximations can be made. In general, one can conclude that since the permeability of soils is small, at high frequencies, the fluid is resisted by viscosity and cannot have a significant velocity relative to solid (Mei and Foda, 1982). But, near the mud line (free surface), fluid can drain, and relative velocity can not be neglected. Near the ground surface, vertical component of $G \nabla^{2} v$ in equation (7.6) is dominant, and inertial terms in comparison are negligible. The boundary layer correction of the solid velocity is irrotational which implies that vertical velocities are much larger than the horizontal ones (Mei and Foda, 1981). In equation (7.7), the last term is neglected near the free surface, and it finally reduces to a diffusion equation in terms of $p$ to be solved for the boundary layer correction. 


$$
k^{*} \frac{\partial^{2} p^{\mathrm{b}}}{\partial y^{2}}=\left[\frac{n}{\beta}+\frac{1-2 v}{2 G(1-v)}\right] \frac{\partial p^{\mathrm{b}}}{\partial t}
$$

The boundary layer thickness is determined from

$$
\delta=\left(\frac{k^{*}}{\omega}\right)^{1 / 2}\left(\frac{n}{\beta}+\frac{1-2 v}{2 G(1-v)}\right)^{-1 / 2}
$$

As seen in equation (7.8a), the thickness of the boundary layer, $\delta$, is very small for small permeability, or high frequency, or large compressibility of water, or large compressibility of the solid matrix. Mei and Foda (1981) have calculated $\delta$ of various earth materials changing from $0.002 \mathrm{~m}$ for granite to $10 \mathrm{~m}$ for coarse sand for $\omega=1 \mathrm{rad} \mathrm{s}^{-1}$.

Solutions obtained for the boundary layer from the solution of equation (7.8) are added to the solutions obtained for the outer region. Using this approximation, Mei and Foda (1981) obtained solutions for progressive waves over a semi-infinite sea bed and a sea bed with finite thickness. In summary, Mei and Foda concluded that for many wave problems, the wave period is much smaller than the consolidation time of soils which in general have low permeability. Thus the relative movement between the fluid and solid is significant only near the free surface of the porous medium ("mudline"). Chen (1986) applied Mei and Foda's (1981) boundary layer theory to study the effect of sediment on earthquake-induced reservoir hydrodynamic response.

Rigid frame analysis of Morse (1952) was extended by Nolle et al. (1963) to allow the bulk modulus of the sand. Nolle et al. stated the equations of motion for solid matrix and fluid by

$$
\begin{aligned}
& -(1-n) \frac{\partial p}{\partial x}=\rho_{\mathrm{s}}(1-n) \frac{\partial v_{x}}{\partial t}+b\left(v_{x}-V_{x}\right) \\
& -n \frac{\partial p}{\partial x}=\rho_{\mathrm{f}} n \frac{\partial V_{x}}{\partial t}+b\left(V_{x}-v_{x}\right)
\end{aligned}
$$

where $v_{x}$ and $V_{x}$ are the velocities of the solid and the fluid, respectively [compare with equations (7.1) and (7.2) with $\sigma_{x x}=\tau_{x y}=\tau_{x z}=0$ due to rigidity of the matrix]. Nolle et al. expressed $b$ by

$$
b=-i \omega n \rho_{\mathrm{f}}(Y-1)+h^{2} \sigma^{*}
$$

where $\omega$ is the angular (circular) frequency, $Y$ is a constant $(>1)$ used to calculate the effective porosity $(=n / Y)$, and $\sigma^{*}$ is the specific flow resistance approximated by

$$
\sigma^{*}=\frac{2 \sqrt{2 \rho_{\mathrm{f}} \mu_{\mathrm{f}} \omega}}{0.12 n d}
$$

where $d$ is the average particle diameter.

Equations (7.9) are solved simultaneously with an equation of continuity 


$$
-\frac{\partial \rho}{\partial t}=\left[\frac{1}{n / \beta_{1}+(1-n) / \beta_{\mathrm{s}}}\right]\left[n \frac{\partial V_{x}}{\partial x}+(1-n) \frac{\partial v_{x}}{\partial x}\right]
$$

where $\beta_{1}$ and $\beta_{\mathrm{s}}$ are the bulk modules of the liquid and the sand grains, respectively. $o$ is the true density of the porous medium. By introducing $\beta_{\mathrm{s}}$, Nolle et al. allowed a finite compressibility for the solid while taking the elastic modulus of the skeleton to be zero. Equation (7.9a) can be compared with equations (6.2) and (7.5).

\subsection{Modifications of the boundary layer theory}

Later, Mynett and Mei (1983) applied the boundary layer theory to study the propagation of earthquake induced Rayleigh waves. The outer region is divided into two regions. The far field is the region at a distance from the structure and the wave length is the characteristic length. The region around the structure is the near field and has the structural dimension as the characteristic length. In the near field, inertial terms are small. Further applications were also given by Mynett and Mei (1982) and Mei and Mynett (1983).

In a later paper, Mei et al. (1985) included the convective component of the acceleration i.e., $n \rho_{\mathrm{f}} U \partial V_{x} / \partial x$ in equation (7.1) and $(1-n) \rho_{\mathrm{s}} U \partial v_{x} / \partial x$ in equation (7.2), on the left hand side of respective equations (similar approach was also taken by Derski, 1978) and assumed $\partial V_{x} / \partial t \ll U \partial V_{x} / \partial x$ and $\partial v_{x} / \partial t \ll U \partial v_{x} / \partial x$ to study the dynamic response of the ground to an air pressure distribution moving along the surface at a constant speed $U$. These approximations were carried out for a steady-state linearization of governing equations. Similarly, in equation (7.5) the convective component $U \partial p / \partial x$ was added to $\partial p / \partial t$, and assumed $\partial p / \partial t \ll U \partial p / \partial x$. The results were given for supersonic $\left(U / V_{\mathrm{c}}>U / V_{\mathrm{s}}>1\right)$, subsonic $\left(1>U / V_{\mathrm{c}}>U / V_{\mathrm{s}}\right)$ and transonic $\left(U / V_{\mathrm{c}}>1>U / V_{\mathrm{s}}\right)$ loads. $U / V_{\mathrm{c}}$ and $U / V_{\mathrm{s}}$ are Mach numbers for compressional and shear waves, respectively.

\subsection{Wave attenuation in marine sediments}

Attenuation of waves in saturated marine sediments is important in seismic studies of these sediments at low frequency range $(1-100 \mathrm{~Hz})$. Acoustic soundings are conducted at a much higher range (up to $100 \mathrm{KHz}$ ). The evaluation of the attenuation of acoustic waves of low amplitude over relatively long distances has been a major interest in geophysics. To develop a unified theory over a wide range of frequencies, Stoll and Bryan (1970) started with Biot's theory [equations (2.49) and (2.50)] to study the attenuation of dilational wave of the first kind. Stoll and Bryan, by casting the parameters $H, \alpha M$, and $M$ of these equations in terms of bulk modulus of the discrete grains, the water, and the solid matrix, and the shear modules of the matrix, demonstrated that attenuation is controlled by the inelasticity of the matrix at low frequencies, and by viscosity of the fluid at higher frequencies. Thus at low frequencies, there is a linear dependence of attenuation on frequency, $f$. At high frequency, attenuation is controlled by $f^{n}$ where $n$ first increases from one to two, and then gradually decreases. At very high frequencies, matrix losses are dominant again, thus causing $n$ to increase. The definition of 
"low" and "high" is a relative term depending on the material. As noted by Stoll and Bryan, fluid losses dominate for granular materials like sand over most of the frequency range due to friction at contact points of grains. For materials like clays, losses are dominated by the solid matrix. Stoll and Bryan (1970) and Stoll (1974) used a functional form of frequency correction factor for high frequencies (Biot, 1956b).

$$
F(\kappa)=\frac{\kappa T(\kappa)}{4(1-2 T(\kappa) / i \kappa)}
$$

where $T(\kappa)$ is given in terms of real and imaginary parts of the Kelvin function

$$
T(\kappa)=\frac{\operatorname{ber}^{\prime}(\kappa)+i \operatorname{ber}^{\prime}(\kappa)}{\operatorname{ber}(\kappa)+i \operatorname{ber}(\kappa)}
$$

and $\kappa$ is defined by

$$
\kappa=a\left(\frac{\omega \rho_{\mathrm{f}}}{\mu_{\mathrm{f}}}\right)^{1 / 2}
$$

where $a$ is the pore size parameter (for circular pores, it is the radius) and $\omega$ is the angular frequency. For low frequencies $F(\kappa)$ approaches to unity.

Stoll (1977) mentioned the significance of the dilatational waves of the second kind in multilayer systems where energy exchanges can occur at interfaces. Plona (1980) has demonstrated the existence of these waves in saturated porous sintered glass. Stoll (1980) noted the non-linear dependence of acoustic properties on cyclic strain amplitude and static stress level. In this study, Stoll developed a mathematical model based on the work of Biot (1956a). Stoll and Kan (1981) have shown the significance differences in the reflection of waves at a fluidsediment interface depending on the type of modeling used to represent the sediment i.e., viscoelastic solid vs. water saturated porous viscoelastic matrix. A porous medium representation should be preferred for high permeability sediments or high frequency sources. Factors affecting the dilatational wave velocity in marine sediment was also investigated by Brandt (1960) by employing his model. Brandt's (1955) model represented the marine and sediments as liquid-saturated aggregate of spherical particles (distinct element model noted in Section 1). A correction factor incorporated the elasticity of pore fluid in an expression to calculate the wave velocity.

McCann and McCann (1969) and Smith (1974) have observed disappearance of solid friction for sediment grains finer than sand. For this type of sediment, the loss mechanism is entirely viscous. As the percentage of clay size particles increases, the effect of relative motion decreases. Then, the frequency dependence becomes quite complex. For very fine grained high porosity sediments of deep oceans, the medium behaves like seawater in its response to frequency variations. 


\section{Application of mixture theory}

Treatment of particulate volume fraction as a constitutive variable in the mixture theory formulation for a multiphase medium like porous materials was introduced by Goodman and Cowin (1972) among others. Application of mixture theory to analyze the wave propagation in a fluid-solid mixture has received limited attention due to complexity of the theoretical exposition and difficulty in relating to practical problems. However, in the last few years, there are a number of papers providing a useful tool and an alternative to deal with wave propagation in porous media. A general treatment of the mixture theory is provided by Bowen (1976).

Raats' publications starting with Raats and Klute (1968) appear to be one of the first studies in this area. Raats has provided a framework for the construction of a mixture theory to study the balance of mass and momentum in porous media. Raats regarded the soil as a mixture of phases with an exchange of momentum taking place in the interfaces between them. Later, Raats (1969) presented an analysis of the propagation of sinusoidal pressure oscillations at a plane boundary into a structured porous medium. Pores of the medium have been classified into two: large and small pores. Raats has found that when the frequency of the oscillation is small, the heterogeneity of the medium is unnoticeable. Raats extended his analysis to include the effect of inertial forces into the jump conditions at the boundaries in addition to introducing an inertial force in the differential balance of forces.

A mixture theory for shock loading of wet tuff was presented by Drumheller (1987). Drumheller's work was a generalization of Herrmann's $(1968,1972)$ model. Drumheller considered an effective stress expression which corresponds to Biot and Willis' (1957) work [equation (2.16)] rather than the original expression of Terzaghi [(equation (4.1a)]. According to the Drumheller's theory, dilatancy occurs when the shear modulus is specified as a function of the porosity, and the np function is universal for all saturation values. Later, Grady et al. (1986) did similar work for dry and water-saturated porous calcite. In earlier works, others, e.g., Garg (1971, 1987), Garg and Kirsch (1973), Morland (1972), and Sawicki and Morland (1985) presented models for a water-saturated porous medium. Their theories similar to that of Bedford and Drumheller's (1979) work, were based on the adaptation of general mixture theory. However, they did not consider intrinsic behavior of immiscible constituents. Garg and Nayfeh (1986) extended the mixture theory approach to unsaturated soils [see equations (5.6)-(5.8)].

Garg (1971) developed a formulation based on the theory of interacting continua for a mixture of a solid and a fluid by defining effective stress and densities in terms of volume fractions of each phase, partial stresses and partial densities. Garg (1971) notes that the attenuation force (diffusive force as he called it) should be a function of partial pressures of each phase for large pressure gradients. Referring to Swift and Kiel (1962), he also suggested to have higher order terms of $(\mathbf{u}-\mathbf{U})$ for larger velocities. Later, Garg et al. (1975) generalized the constitutive relations of Garg (1971) and Morland (1972) to include thermodynamic effects. They solved the proposed model to study the shock wave propagation in tuff- 
water mixture. Their numerical results indicate an increase in pulse rise time with increasing permeability.

Density variations in an inhomogeneous granular solid were considered in a mixture theory formulation developed by Nunziato and Walsh (1977) based upon concepts developed by Goodman and Cowin (1972). Later, Nunziato et al. (1978) applied their model to study one-dimensional wave propagation in an explosive material.

Bowen (1976) considered the saturated porous medium as a binary mixture of a linear elastic fluid and a linear elastic solid. Bowen and Reinicke (1978) stated four governing differential equations for displacements and temperatures of each phase, and they have shown that when there is momentum transfer between phases, there is only one mode of non-dispersive propagation in the low frequency range independent of the energy transfer. However, phase velocities and the attenuation coefficients depend on the presence of energy transfer between the phases. Thermal effects on wave propagation were also studied by Pecker and Deresiewicz (1973). Pecker and Deresiewicz have determined four distinct dilatational motions. The first two represent modifications of fast and slow waves (first and second $\mathrm{P}$ waves) at constant temperatures, and the other two are diffusion type modes similar to the thermal waves in a single-phase thermoplastic solid. Jones and Nur (1983) have observed that shear velocity and attenuation decrease with increasing temperature at all pressures in a saturated rock. In frozen soils, wave attenuation from low-level impact was found to be exponential (Dutta et al., 1990). Later, Bowen (1982) extended his mixture theory analysis (Bowen, 1980) to compressible porous media. Bowen compared his model to the one proposed by Biot (1962a). Bowen and Lockett (1982) have shown that longterm inertial effects cannot be neglected under certain circumstances such as the occurrence of resonance displacements for a harmonically varying compression at some loading frequencies. Neglecting inertia does not predict this type of behavior. We should note that in long-term diffusion type slow processes, the inertia terms have been generally neglected. Inertia terms were considered important at small times. We refer to Zienkiewicz and Bettess (1982c) as an example of this type of work, (see Section 3.3).

Katsube (1985) investigated Biot's constitutive relations by modifying Carroll's (1980) developments. Katsube and Carroll (1987) modified the mixture theory of Green and Naghdi (1965) and applied to porous media. They compared the resulting theory with Biot's theory and concluded that they are equivalent when fluid velocity gradients are ignored. Liu and Katsube (1990) predicted the existence of a second kind of a shear wave using the mixture theory of Crochet and Naghdi (1966). This wave is caused by the skew-symmetric portions of the partial stress tensors in the mixture theory of Crochet and Naghdi. Pride et. al. (1992) used local volume averaging technique in the derivation and argued that interaction torques caused by the skew-symmetrix portions should not be expected.

Loret (1990) and, Loret and Pervost (1991) studied dynamic strain localization in saturated porous media. Boer et al. (1993) formulated the field equations assuming both fluid and solid constituents are incompressible and obtained an analytical solution for transient wave propagation. 
By employing the theory of mixtures and assuming that the mixture consists of two non-polar, incompressible constituents, Prevost (1980) obtained the conservation of mass and momentum equations for the solid and the fluid phases as

$$
\begin{aligned}
& \frac{\partial n}{\partial t}+(1-n) \nabla \cdot v=0 \\
& \nabla \cdot[n(V-v)]+\nabla \cdot v=0 \\
& \nabla \cdot \sigma^{\prime}-(1-n) \nabla p+n^{2} \rho_{\mathrm{f}} k^{-1}(v-V)+(1-n) \rho_{\mathrm{s}} b=(1-n) \rho_{\mathrm{s}} \frac{d_{\mathrm{s}} v}{d t} \\
& n \rho_{\mathrm{f}}(v-V): \nabla V-n \nabla p-n^{2} \rho_{\mathrm{f}} k^{-1}(v-V)+\rho_{\mathrm{f}} n b=n \rho_{\mathrm{f}} \frac{d_{\mathrm{s}} V}{d t}
\end{aligned}
$$

where $d_{\mathrm{s}} / d t$ is the material derivative with respect to moving solid phase. In deriving these equations Prevost assumed that since there is no moment of momentum supply between the two phases, the partial stress tensors for both phases are symmetric. It was also assumed that the fluid has no average shear viscosity. Later Prevost $(1983,1984,1985)$ solved these equations by using a finite element technique.

Hsieh and Yew (1973) accounted for the change in porosity in their mixture theory formulation by expressing the porosity, $n$ as

$$
n=n_{\mathrm{o}}+\Delta n
$$

where $n_{\mathrm{o}}$ and $u n$ are the initial porosity and small incremental change in porosity, respectively. Furthermore, the relationship among the pore fluid pressure, $p$, dilatation, $\epsilon$, and $\Delta n$ is expressed by

$$
-p=Q^{*} \epsilon-N^{*} \Delta n
$$

where coefficients $Q^{*}$ and $N^{*}$ which should be determined experimentally, do not correspond to Biot coefficients [see equation (2.16)]. Hsieh and Yew (1973) presented a numerical solution for the dilatational and rotational waves.

As noted in Section 5, Berryman (1988) presented a mixture theory for unsaturated porous media. Berryman published his theory in a series of papers which deal with different aspects of the problem such as inhomogeneity and normalization constraint (e.g., Berryman and Milton, 1985; Berryman, 1985).

In Section 2.7, we noted that Biot's theory does not take into account the timedependent pore collapse of a porous matrix. However, dry porous materials, such as granular high energy solid propellants, granular explosives, dry metal powders exhibit pore crushing and pore collapse. Carroll and Holt (1972) and Butcher et al. (1974) described a time-dependent pore collapse mechanism for porous aluminum. Baer and Nunziato (1986), Baer (1988), Gokhale and Krier (1982) and Powers et al. (1989) provided two-phase continuum mixture equations to describe the motion of a mixture of solid particles and gas. These equations simulate the deflagration-to-detonation transition in a column of granular explosives. Powers 
et al. (1989) stated these equations by neglecting the effects of diffusive momentum and energy transport, and the compaction work

$$
\begin{aligned}
& \frac{\partial \rho_{i} \phi_{i}}{\partial t}+\frac{\partial\left(\rho_{i} \phi_{i} v_{i}\right)}{\partial x}=A_{i} \\
& \frac{\partial \rho_{i} \phi_{i} v_{i}}{\partial t}+\frac{\partial\left(p_{i} \phi_{i}+\rho_{i} \phi_{i} v_{i}^{2}\right)}{\partial x}=B_{i} \\
& \frac{\partial}{\partial t}\left[\rho_{i} \phi_{i}\left(e_{i}+\frac{v_{i}^{2}}{2}\right)\right]+\frac{\partial}{\partial t}\left[\rho_{i} \phi_{i} u_{i}\left(e_{i}+\frac{v_{i}^{2}}{2}+\frac{p_{i}}{\rho_{i}}\right)\right]=C_{i} \\
& \frac{\partial \phi_{2}}{\partial t}+v_{2} \frac{\partial \phi_{2}}{\partial x}=\phi_{1} \frac{\phi_{2}}{\mu_{c}}\left[p_{2}-p_{1}-\sigma^{\prime}\left(\phi_{2}\right)\right]
\end{aligned}
$$

where $\rho_{i}$ is the density, $p_{i}$ is the pressure, $e_{i}$ is the energy, $v_{i}$ is the velocity, $d_{i}$ is the volume fraction for each phase ( $i=1$ for the gas, $i=2$ for the solid). Equations (8.7)-(8.9) are the balance equations for mass, momentum and energy of each phase. Interphase transport is represented by $A_{i}, B_{i}$, and $C_{i}$ which are functions of other parameters such as densities, velocities, and pressures of each phase. By definition, the sum of each term is equal to zero, i.e., $A_{1}+A_{2}=0$. Equation (8.10) similar to Butcher et al.'s (1974) pore collapse equation, is the "compaction equation" where $m_{\mathrm{c}}$ is the "compaction viscosity" and $s^{\prime}$ is the intergranular stress expressed as a function of volume fraction. Different phases of compaction, i.e., elastic, plastic, would generate different $s^{\prime}$ expressions (Carroll and Holt, 1972). Substitution of equation (8.10) into equations (8.7)-(8.9) would yield hyperbolic equations (Baer and Nunziato, 1986). State expressions will express $p_{i}$ and $e_{i}$ in terms of $\left(\rho_{i}, T_{i}\right)$ and $\left(p_{i}, \rho_{i}\right)$ respectively. $T_{i}$ is the phase temperature. By definition $d_{1}+d_{2}=1$. Powers et al.'s (1987) model admits both subsonic and supersonic compaction waves. They have shown that when compaction waves travel faster than the ambient sound speed of the solid, a shock preceding the compaction wave structure is expected. There was no leading shock for subsonic compaction waves.

Beskos (1989) studied the dynamic behavior of fluid saturated fissured rocks. Beskos developed his model along the lines of the theory of mixture formulation of Aifantis (1979) and, Wilson and Aifantis (1984) in a notational framework similar to the one employed by Vardoulakis and Beskos (1986). In companion papers, Beskos et al. (1989a, 1989b) studied the propagation of harmonic body and Rayleigh waves. Their analysis reveals the existence of three dilatational (compressional) waves and one rotational (shear) wave. The presence of fissures results in the appearance of an additional dilatational wave in a fissured porous medium.

Another approach of formulating multiphase equations is local volume averaging. This approach started after the development of the theorem for the local volume average of a gradient (Slattery, 1967; Anderson and Jackson, 1967; Whitaker, 1967). It has been recently applied to wave propagation problems. de la Cruz and Spanos (1985) made the first attempt to rederive Biot's theory. In a later 
paper, de la Cruz and Spanos (1989) extended their theory to include thermodynamical coupling. Garg (1987) developed the complete set of balance laws for multiphase media. However, in all these works the main problem was the determination of the constitutive relations in terms of averaged variables. Recently, Pride et al. (1992) rederived Biot's equations and obtained the same expressions for the coefficients in Biot's theory. An alternative approach of homogenization is the two-space method. It was first developed and studied by SanchezPalancia (1980) and Keller (1977). It was applied to wave propagation by Burridge and Keller (1981), Levy (1979), and Auriault (1980, 1985). In principle both local volume averaging and two-space method yield the same results. However, application of local volume averaging is simpler and enables physical interpretations of the averaged expressions.

\section{The use of macroscopic balance equations to obtain wave propagation equations in saturated porous media}

In this section we will develop the governing equations for wave propagation in a saturated compressible porous medium from the macroscopic momentum and mass balance equations for both the solid matrix and fluid phase. The equations are written for an elastic solid matrix and a Newtonian compressible fluid that completely fills the void space. The constitutive equations for the elastic solid matrix are written in terms of the effective stresses. The resulting governing equations are in terms of fluid and solid velocities, effective stresses, displacements, fluid pressure, fluid density, and porosity. This approach has been presented by Bear and Corapcioglu (1989).

We assume that the compressible porous medium is fully saturated by a singlephase, single-component fluid. As a result of dynamic loading, stresses in the fluid change. This is accompanied by a corresponding change in the effective stresses in the solid matrix. A change in effective stress produces the deformation of the porous medium.

The approach we present in this chapter offers an alternative methodology to obtain the wave propagation equations. As opposed to Biot's approach which employs the kinetic energy density functions and dissipation energy functions, we state the conservation of momentum and mass equations to formulate the problem.

\subsection{Mass balance equations for the fluid and the solid matrix}

We start from the three-dimensional mass balance equation for a fluid that saturates a porous medium (e.g., Bear and Corapcioglu, 1981)

$$
\nabla \cdot\left(\rho_{\mathrm{f}} n V_{\mathrm{f}}\right)+\frac{\partial\left(\rho_{\mathrm{f}} n\right)}{\partial t}=0
$$

where $V_{\mathrm{f}}$ is the mass-weighted velocity of the fluid, $\rho_{\mathrm{f}}$ is the density of the fluid, and $n$ is the porosity of the medium. In deriving (9.1), we have neglected the 
dispersive mass flux due to spatial variations in the fluid's density. Similarly, the balance equation for the solid mass can be written as

$$
\nabla \cdot\left(\rho_{\mathrm{s}}(1-n) V_{\mathrm{s}}\right)+\frac{\partial\left(\rho_{\mathrm{s}}(1-n)\right)}{\partial t}=0
$$

where $V_{\mathrm{s}}$ is the mass-weighted velocity of the solid due to deformation, $\rho_{\mathrm{s}}$ is the density of the solid. By introducing the definition of material derivative with respect to the moving solid particles $D_{\mathrm{s}}() / D t$, and assuming that the solid's density is constant, equation (9.2) can be expressed by (Bear and Corapcioglu, 1981)

$$
\nabla \cdot V_{\mathrm{s}}=\frac{1}{1-n} \frac{D_{\mathrm{s}}(1-n)}{D t}
$$

The mass balance equation for the fluid phase can be rewritten in a different form by making use of equation (9.3) (Bear and Corapcioglu, 1981).

$$
\rho_{\mathrm{f}} \nabla \cdot n\left(V_{\mathrm{f}}-V_{\mathrm{s}}\right)+n \frac{D_{\mathrm{f}} \rho_{\mathrm{f}}}{D t}+\rho_{\mathrm{f}} \frac{D_{\mathrm{s}} n}{D t}+\rho_{\mathrm{f}} n \nabla \cdot V_{\mathrm{s}}=0
$$

where $D_{\mathrm{f}}() / D t$ is the material derivative with respect to an observer moving with the fluid.

\subsection{Momentum balance equations for the fluid and solid phases}

Macroscopic momentum balance equations for the fluid can be obtained by neglecting certain dispersive terms in the averaging process, in the form (Bear and Bachmat, 1984)

$$
n \rho_{\mathrm{f}} \frac{D_{\mathrm{f}} V_{\mathrm{f}}}{D_{\mathrm{t}}}=\nabla \cdot n \sigma_{\mathrm{f}}+n \rho_{\mathrm{f}} F+\frac{1}{U_{0}} \int_{S_{\mathrm{fs}}} \sigma_{\mathrm{f}} \cdot v_{\mathrm{f}} d S
$$

Similarly, for the solid matrix

$$
(1-n) \rho_{\mathrm{s}} \frac{D_{\mathrm{s}} V_{\mathrm{s}}}{D_{\mathrm{t}}}=\nabla \cdot(1-n) \sigma_{\mathrm{s}}+(1-n) \rho_{\mathrm{s}} F+\frac{1}{U_{0}} \int_{S_{\mathrm{fs}}} \sigma_{\mathrm{s}} \cdot v_{\mathrm{s}} d S
$$

where $\sigma_{\mathrm{f}}$ and $\sigma_{\mathrm{s}}$ are the stress tensors in the fluid and solid phases, respectively, $F$ is the body force per unit mass, equal to the gravitational acceleration $g(=$ $-g \nabla z)$ where $z$ is the vertical coordinate, $U_{0}$ is the volume of a representative elementary volume, $S_{\mathrm{fs}}$ is the contact area between the solid and fluid phases within the representative elementary volume and $n_{\mathrm{f}}$ and $n_{\mathrm{s}}$ are the unit outward vectors on the interphase boundaries between them. The terms on the left hand side of equations (9.4) and (9.5) represent the inertial force per unit volume. The first two terms on the right hand side represent the stress and the body forces, respectively. The last terms in equations (9.4) and (9.5), represent the interfacial momentum transfer from the fluid phase to the solid phase and vice-versa. Their sum should vanish. 
By adding equations (9.4) and (9.5), we obtain the momentum balance equation for the porous medium as a whole

$$
n \rho_{\mathrm{f}} \frac{D_{\mathrm{f}} V_{\mathrm{f}}}{D t}+(1-n) \rho_{\mathrm{s}} \frac{D_{\mathrm{s}} V_{\mathrm{s}}}{D t}=\nabla \cdot \sigma+\left[n \rho_{\mathrm{f}}+(1-n) \rho_{\mathrm{s}}\right] g \nabla z
$$

where $\sigma$ is the total stress tensor, expressed as

$$
\sigma=(1-n) \sigma_{\mathrm{s}}+n \sigma_{\mathrm{f}}
$$

As we noted earlier each solid grain is assumed incompressible. The total stress is related to the effective stress, $\sigma_{s}^{\prime}$, and to the stress in the fluid, $\sigma_{\mathrm{f}}$, by

$$
\sigma=(1-n)\left(\sigma_{\mathrm{s}}-\sigma_{\mathrm{f}}\right)+\sigma_{\mathrm{f}}=\sigma_{\mathrm{s}}^{\prime}+\sigma_{\mathrm{f}}
$$

In writing equation (9.8), we assume that solid matrix deformation is caused only by the stress in the solid matrix minus the isotropic effect of the fluid pressure surrounding each grain (e.g., Bear, Corapcioglu and Balakrishna, 1984). In soil mechanics, $\sigma_{\mathrm{s}}^{\prime}$ corresponds to Terzaghi's definition of effective stress. When grain compressibility is taken into account, Verruijt (1984) has shown that

$$
\sigma=\sigma_{\mathrm{s}}^{\prime}+(1-\gamma) \sigma_{\mathrm{f}}
$$

where $\gamma$ is the ratio between the solid's compressibility and that of the soil. We shall assume that $\gamma \ll 1$.

Since Darcy's law expresses the mass weighted velocity of the fluid relative to the solid

$$
n\left(V_{\mathrm{f}}-V_{\mathrm{s}}\right)=n V_{\mathrm{r}}=\frac{K}{\mu_{\mathrm{f}}}\left(\nabla p+\rho_{\mathrm{f}} g \nabla z\right)
$$

It is convenient to rewrite equation (9.6) as

$$
\begin{aligned}
n \rho_{\mathrm{f}} & \frac{D_{\mathrm{r}} V_{\mathrm{r}}}{D t}+\left[(1-n) \rho_{\mathrm{s}}+n \rho_{\mathrm{f}}\right] \frac{D_{\mathrm{s}} V_{\mathrm{s}}}{D t}+\left[V_{\mathrm{s}}: \nabla V_{\mathrm{s}}+V_{\mathrm{r}}: \nabla V_{\mathrm{s}}\right] n \rho_{\mathrm{f}} \\
& =\nabla \cdot \sigma+\left[(1-n) \rho_{\mathrm{s}}+n \rho_{\mathrm{f}}\right] g \nabla^{\prime} z
\end{aligned}
$$

The stress in the fluid phase can be divided into two components, i.e., the viscous shear, $\tau_{\mathrm{f}}$, and the average fluid pressure, $p$

$$
\sigma_{\mathrm{f}}=\tau_{\mathrm{f}}-p I
$$

where $I$ is the unit tensor. For a Newtonian compressible fluid, the constitutive equation takes the form

$$
\tau_{\mathrm{f}}=\mu_{\mathrm{f}}\left(\nabla V_{\mathrm{f}}+\left(\nabla V_{\mathrm{f}}\right)^{\mathrm{T}}\right)+\lambda_{\mathrm{f}} \nabla \cdot V_{\mathrm{f}} I
$$

where $\mu_{\mathrm{f}}$ and $\lambda_{\mathrm{f}}$ are the fluid's dynamic and bulk viscosities. For an incompressible Newtonian fluid, the second term on the right hand side can be neglected. Similarly, for an isotropic, perfectly elastic solid matrix, the stress-strain relationship for the solid matrix assumed to take the form

$$
\sigma_{\mathrm{s}}^{\prime}=G\left(\nabla W+(\nabla W)^{\mathrm{T}}\right)+\lambda \nabla \cdot W I
$$


where $W$ is the displacement vector and $G$ (shear modulus) and $\lambda$ are the Lame constants. Note that by definition

$$
V_{\mathrm{s}}=\frac{D_{\mathrm{s}} W}{D t}
$$

Equation (9.12) can be written for an incompressible Newtonian fluid in terms of the relative velocity as

$$
\tau_{\mathrm{f}}=\mu_{\mathrm{f}}\left(\nabla V_{\mathrm{r}}+\left(\nabla V_{\mathrm{r}}\right)^{\mathbf{T}}+\nabla V_{\mathrm{s}}+\left(\nabla V_{\mathrm{s}}\right)^{\mathrm{T}}\right)
$$

The integral that expresses the momentum transfer from the solid to the fluid through their common surface, $S_{\mathrm{fs}}$, per unit volume of porous medium can be expressed as

$$
\frac{1}{U_{0}} \int_{S_{\mathrm{fs}}} \tau_{\mathrm{f}} \cdot v_{\mathrm{f}} d S=-\mu_{\mathrm{f}} b_{\mathrm{f}} \cdot n V_{\mathrm{r}}
$$

where $b_{\mathrm{f}}$ is a macroscopic coefficient representing the effect of the microscopic configuration of the $S_{\mathrm{fs}}$ surface. It is related to a shape factor and the hydraulic radius of the void space (Bear and Bachmat, 1984). The substitution of equations (11), (14) and (15) into equation (4) yields ?????? where the tortuosity, $T_{\mathrm{f}}$ is a second rank symmetric tensor which is related to the geometric features of the microscopic distribution of the fluid phase in the vicinity of a point (Bear and Bachmat, 1984). If we neglect the effect of internal viscous resistance to the flow in the fluid

$$
\begin{aligned}
& n \rho_{\mathrm{f}} \frac{D_{\mathrm{f}} V_{\mathrm{f}}}{D t}-\mu_{\mathrm{f}} \nabla \cdot\left\{n\left(\nabla V_{\mathrm{r}}+\left(\nabla V_{\mathrm{r}}\right)^{\mathrm{T}}+\nabla V_{\mathrm{s}}+\left(\nabla V_{\mathrm{s}}\right)^{\mathrm{T}}\right)\right\} \\
& \quad+T_{\mathrm{f}}\left(\nabla p+\rho_{\mathrm{f}} g \nabla z\right)+\mu_{\mathrm{f}} b_{\mathrm{f}} \cdot n V_{\mathrm{r}}
\end{aligned}
$$

(i.e., $\nabla . n t_{\mathrm{f}}=0$ ), we obtain

$$
\rho_{\mathrm{f}} T_{\mathrm{f}}^{-1} \frac{D_{\mathrm{f}} V_{\mathrm{f}}}{D t}+\nabla p+\rho_{\mathrm{f}} g \nabla z+n \mu_{\mathrm{f}} K^{-1} V_{\mathrm{r}}=0
$$

where $K$ is the permeability of the porous medium

$$
K=n T_{\mathrm{f}} b_{\mathrm{f}}^{-1}
$$

The momentum balance equation for the saturated porous medium as a whole can be obtained from equation (6) by substituting equations (8) and (11) and by neglecting the effect of internal friction as

$$
n \rho_{\mathrm{s}} \frac{D_{\mathrm{f}} V_{\mathrm{f}}}{D t}+(1-n) \rho_{\mathrm{s}} \frac{D_{\mathrm{s}} V_{\mathrm{s}}}{D t}=\nabla \cdot \sigma_{\mathrm{s}}^{\prime}-\nabla p-\left(n \rho_{\mathrm{f}}+(1-n) \rho_{\mathrm{s}}\right) g \nabla z
$$




\subsection{Complete set of equations}

If we summarize the complete set of three-dimensional governing equations in terms of $V_{\mathrm{f}}$ and $V_{\mathrm{s}}$ (note that $V_{\mathrm{r}}=V_{\mathrm{f}}-V_{\mathrm{s}}$ ), we can list the following seventeen equations in a three dimensional system

- one mass balance equation for the fluid, equation (9.1) or (9.3a)

- one mass balance equation for the solid, equation (9.2) or (9.3)

- three momentum balance equations for the fluid, equation (9.17)

- three momentum balance equations for the saturated porous medium, equation (9.19)

- three equations defining the solid's velocity, equation (9.13a)

- six constitutive equations for an elastic solid matrix, equation (9.13)

for seventeen unknowns, i.e., three fluid velocities, $V_{\mathrm{f}}$; three solid velocities, $V_{\mathrm{s}}$; six effective stress components, $\sigma_{\mathrm{s}}$; three displacements, $W$; porosity, $n$; and pore fluid pressure, $p$. Note that so far, we assumed a constant fluid and solid particle densities. If we assume a compressible fluid, then we need an additional constitutive equation to relate $\rho_{\mathrm{f}}$ to $p$.

As noted by Bear and Corapcioglu (1989), some simplifications can be introduced to the general formulation to show the derivation of various well-known equations. For example, if we neglect the inertial terms in equation (9.16), we obtain Darcy's law. Equilibrium equations satisfying the total stress field can be obtained from equation (9.19) by dropping the inertial terms. The resulting set of equations obtained by deleting the inertial terms in equations (9.17) and (9.19), constitutes the three-dimensional consolidation model of Biot (1941). This corresponds to "very slow phenomena" where all acceleration forces can be neglected (Zienkiewicz and Bettess, 1982c). "Very rapid phenomena" occur when the permeability becomes very small. Zienkiewicz and Bettess consider a case in which the acceleration in the fluid is neglected ("medium speed phenomena) (see Section 3.3). In this case, we define the fluid velocity $V_{\mathrm{f}}$ in terms of a fluid displacement $W_{\mathrm{f}}, V_{\mathrm{f}}=\partial W_{\mathrm{f}} / \partial t$ by neglecting the convective term in the material derivative $D_{\mathrm{f}} W_{\mathrm{f}} / D t$.

\section{Wave propagation in fractured porous media saturated by two immiscible fluids}

The single-porosity models are shown to be fairly successful to describe the behavior of porous materials. However, they are not suitable for fractured (or fissured) porous materials. In such systems, although most of the fluid mass is stored in the pores, the fracture permeability is much higher than the permeability of the pores. This leads to two distinct pressure fields: one in the fractures and the other in the pores. Barenblatt et al. (1960) appear to be the first researchers proposing a double-porosity model to represent naturally fractured porous media. A double-porosity model can be considered as a three phase system, i.e., solid 
phase, fluid phase in the pores and fluid phase in the fractures, with fluid mass exchange between the pores and fractures.

Although flow in fractured porous media has been studied extensively (e.g., Barenblatt et al., 1960; Barenblatt, 1963; Bear and Berkowitz, 1987), there is limited work in deformable fractured porous media. Duguid and Lee (1977) considered incompressible solid grains and used double-porosity concept in the formulation. They simplified the governing equations by neglecting solid displacement from the flow equations and used the finite element method for numerical analysis. Aifantis and his co-workers published a series of papers on consolidation of saturated fractured porous media (Wilson and Aifantis, 1982; Beskos and Aifantis, 1986; Khaled et al. 1984). The final set of equations is a direct generalization of Biot's consolidation theory. The phenomenological coefficients of the theory were expressed in terms of measurable quantities by Wilson and Aifantis (1982). Uniqueness and some general solutions were presented by Beskos and Aifantis (1986). Khaled et al. (1984) employed the finite element method to solve the governing equations for some practical problems. They reduced the number of coefficients from fifteen to nine by simply "physically motivated arguments". Wilson and Aifantis (1984) extended Aifantis' work and studied wave propagation in saturated fractured porous media without detailed derivations. Their analysis showed three compressional waves. Similar results were obtained by Beskos who published a series of papers on the dynamics of fissured rocks (Beskos, 1989; Beskos et al. 1989a; Beskos et al., 1989b). Beskos (1989) assumed that the medium is linearly elastic. However, the effect of fluid pressure on the deformation of matrix was not considered. Beskos connected this to the definition of partial stresses. But when we assume that there is no relative movement between solid and fluid phases, Beskos' equation for the deformation of solid phase uncouples from fluid pressures. Bear and Berkowitz (1987) suggested a set of constitutive relations to model quasi-static behavior of fractured porous media. They assumed that the changes in the volume fraction of pores and fractures are linear functions of incremental pressures.

Tuncay and Corapcioglu (1996a) presented a theory of wave propagation in fractured porous media based on the double-porosity concept. The macroscopic constitutive relations, and mass and momentum balance equations were obtained by volume averaging the micro-scale balance and constitutive equations and assuming small deformations. In micro-scale the grains were assumed to be linearly elastic and the fluids are Newtonian. Momentum transfer terms were expressed in terms of intrinsic and relative permeabilities assuming the validity of Darcy's law in fractured porous media. The macroscopic constitutive relations of elastic porous media saturated by one or two fluids and saturated fractured porous media were obtained from the constitutive relations they developed. The macroscopic constitutive relations contain the bulk modulus of the fractured porous medium, bulk modulus of the nonfractured medium, bulk modulus of the solid grains and shear modulus of the solid matrix. The capillary pressure effects were taken into account by assuming the validity of the relationship between capillary pressure and saturation. The momentum transfer terms are expressed in terms of intrinsic 
and relative permeabilities as a first order approximation. The final set of equations has an hyperbolic behavior with dissipation due to interaction terms. In the simplest case, the final set of governing equations reduce to Biot's equations containing the same parameters as of Biot and Willis (1957).

In their derivation, Tuncay and Corapcioglu (1996a) assumed immiscible fluid phases at rest. Furthermore, the solid phase is assumed to be isotropic, experiencing small deformations and providing all shear resistance of the porous medium. Momentum transfer terms are expressed in terms of intrinsic and relative permeabilities assuming the validity of Darcy's law in fractured porous media. The theory is limited to low frequency wave propagation. The medium is assumed to have fractures which are referred as secondary pores. The pores of the nonfractured part of the porous medium are referred as primary pores. The secondary pores are saturated by wetting fluid whereas the primary pores are saturated by wetting and non-wetting fluids. The mass transfer between the primary pores and secondary pores per unit volume is approximated by (Tuncay and Corapcioglu, 1996b)

$$
\frac{1}{V} \int_{S_{2 \mathrm{f}}} \rho_{2}\left(u-v_{2}\right) \cdot n_{2} d A=-\frac{1}{V} \int_{S_{\mathrm{f} 2}} \rho_{2}\left(u-v_{\mathrm{f}}\right) \cdot n_{\mathrm{f}} d A=R \rho_{2}\left(\bar{P}_{\mathrm{f}}-\bar{P}_{2}\right)
$$

where $R$ is a material property of the porous medium and the wetting fluid, $\bar{P}_{2}$ and $\vec{P}_{\mathrm{f}}$ are the pressures in fluid phase 2 and phase $f$, respectively. From now on, subscripts $s, 1,2$ and $f$ will refer to the solid phase, non-wetting phase, wetting phase in the primary pores, and the fluid phase in the fractures, respectively. Barenblatt et al. (1960) proposed $R$ as

$$
R=\frac{c K_{\mathrm{f}} A^{2}}{\mu_{2}}
$$

where $A$ is the area of fracture-block contact per unit volume, $c$ denotes a dimensionless shape factor of the fractured medium and $K_{\mathrm{f}}$ is the permeability of the fractures. The macroscopic constitutive relations were obtained as

$$
\begin{gathered}
\alpha_{\mathrm{s}} \bar{\tau}_{\mathrm{s}}=\left(a_{11} \nabla \cdot \bar{u}_{\mathrm{s}}+a_{12} \nabla \cdot \bar{u}_{1}+a_{13} \nabla \cdot \bar{u}_{2}+a_{14} \nabla \cdot \bar{u}_{\mathrm{f}}+\left(\frac{a_{14}}{\alpha_{\mathrm{f}}}-\frac{a_{13}}{\alpha_{2}}\right) M\right) I+ \\
G_{\mathrm{fr}}\left(\nabla \bar{u}_{\mathrm{s}}+\left(\nabla \bar{u}_{\mathrm{s}}\right)^{T}-\frac{2}{3} \nabla \cdot \bar{u}_{\mathrm{s}}\right) \\
\alpha_{1} \bar{P}_{1}=a_{21}+a_{22} \nabla \cdot \bar{u}_{\mathrm{s}} \nabla \cdot \bar{u}_{1}+a_{23} \nabla \cdot \bar{u}_{2}+a_{24} \nabla \cdot \bar{u}_{\mathrm{f}}+\left(\frac{a_{24}}{\alpha_{\mathrm{f}}}-\frac{a_{23}}{\alpha_{2}}\right) M \\
\alpha_{2} \bar{P}_{2}=a_{31} \nabla \cdot \bar{u}_{\mathrm{s}}+a_{32} \nabla \cdot \bar{u}_{1}+a_{33} \nabla \cdot \bar{u}_{2}+a_{34} \nabla \cdot \bar{u}_{\mathrm{f}}+\left(\frac{a_{34}}{\alpha_{\mathrm{f}}}-\frac{a_{33}}{\alpha_{2}}\right) M \\
\alpha_{\mathrm{f}} \bar{P}_{\mathrm{f}}=a_{41} \nabla \cdot \bar{u}_{\mathrm{s}}+a_{42} \nabla \cdot \bar{u}_{1}+a_{43} \nabla \cdot \bar{u}_{2}+a_{44} \nabla \cdot \bar{u}_{\mathrm{f}}+\left(\frac{a_{44}}{\alpha_{\mathrm{f}}}-\frac{a_{43}}{\alpha_{2}}\right) M
\end{gathered}
$$

The reader is referred to Tuncay and Corapcioglu (1995a) for expressions of $a_{i j}$ 
in terms of material parameters: $K_{\mathrm{s}}$, bulk modulus of the solid grains; $K_{\mathrm{f}}$, bulk modulus of the fractured porous medium; $K_{\mathrm{fr}}^{\mathrm{m}}$, bulk modulus of the nonfractured porous medium; $\alpha_{\mathrm{i}}$, volume fraction of phase $i ; S_{1}$, saturation of the non-wetting fluid phase; $K_{1}$, bulk modulus of the non-wetting fluid phase; $K_{2}$, bulk modulus of the wetting fluid phase; $P_{\text {cap }}^{\prime}$, derivative of the capillary pressure-saturation relation with respect to $S_{1} ; G_{\mathrm{fr}}$, shear modulus of the solid matrix and $F$, a material propertiy associated with the changes in volume fraction of fractures. $M$ is given by

$$
\frac{\partial M}{\partial t}=R\left(\bar{P}_{\mathrm{f}}-\bar{P}_{2}\right)
$$

We note that $\rho_{2} \partial M / \partial t$ is equal to the mass transfer rate of wetting fluid phase between the primary pores and fractures. The volume averaged momentum balance equations are obtained as

$$
\begin{aligned}
\left\langle\rho_{\mathrm{s}}\right\rangle \frac{\partial^{2} \bar{u}_{\mathrm{s}}}{\partial t^{2}}= & \nabla\left(\left(a_{11}+\frac{G_{\mathrm{fr}}}{3}\right) \nabla \cdot \bar{u}_{\mathrm{s}}+a_{12} \nabla \cdot \bar{u}_{1}+a_{13} \nabla \cdot \bar{u}_{2}+a_{14} \nabla \cdot \bar{u}_{\mathrm{f}}\right. \\
& \left.+\left(\frac{a_{14}}{\alpha_{\mathrm{f}}}-\frac{a_{13}}{\alpha_{2}}\right) M\right)+\nabla \cdot\left(G_{\mathrm{fr}} \nabla \bar{u}_{\mathrm{s}}\right)+C_{1}\left(\bar{u}_{1}-\bar{v}_{\mathrm{s}}\right) \\
& +C_{2}\left(\bar{v}_{2}-\bar{v}_{\mathrm{s}}\right)+C_{3}\left(\bar{v}_{\mathrm{f}}-\bar{v}_{\mathrm{s}}\right) \\
\left\langle\rho_{1}\right\rangle \frac{\partial^{2} \bar{u}_{1}}{\partial t^{2}}= & \nabla\left(a_{21} \nabla \cdot \bar{u}_{\mathrm{s}}+a_{22} \nabla \cdot \bar{u}_{1}+a_{23} \nabla \cdot \bar{u}_{2}+a_{24} \nabla \cdot \bar{u}_{\mathrm{f}}\right. \\
& \left.+\left(\frac{a_{24}}{\alpha_{\mathrm{f}}}-\frac{a_{23}}{\alpha_{2}}\right) M\right)-C_{1}\left(\bar{v}_{1}-\bar{v}_{\mathrm{s}}\right) \\
\left\langle\rho_{2}\right\rangle \frac{\partial^{2} \bar{u}_{2}}{\partial t^{2}=} & \nabla\left(a_{31} \nabla \cdot \bar{u}_{\mathrm{s}}+a_{32} \nabla \cdot \bar{u}_{1}+a_{33} \nabla \cdot \bar{u}_{2}+a_{34} \nabla \cdot \bar{u}_{\mathrm{f}}+\left(\frac{a_{34}}{\alpha_{\mathrm{f}}}-\frac{a_{33}}{\alpha_{2}}\right) M\right) \\
& -C_{1}\left(\bar{v}_{2}-\bar{v}_{\mathrm{s}}\right) \\
\left\langle\rho_{\mathrm{f}}\right\rangle \frac{\partial^{2} \bar{u}_{\mathrm{f}}}{\partial t^{2}}= & \nabla\left(a_{41} \nabla \cdot \bar{u}_{\mathrm{s}}+a_{42} \nabla \cdot \bar{u}_{1}+a_{43} \nabla \cdot \bar{u}_{2}+a_{44} \nabla \cdot \bar{u}_{\mathrm{f}}+\left(\frac{a_{44}}{\alpha_{\mathrm{f}}}-\frac{a_{43}}{\alpha_{2}}\right) M\right) \\
& -C_{3}\left(\bar{v}_{\mathrm{f}}-\bar{v}_{\mathrm{s}}\right)
\end{aligned}
$$

where

$$
\begin{aligned}
& C_{1}=\frac{\left(1-\alpha_{\mathrm{s}}-\alpha_{\mathrm{f}}\right)^{2} S_{1}^{2} \mu_{1}}{K_{\mathrm{p}} k_{r 1}} \\
& C_{2}=\frac{\left(1-\alpha_{\mathrm{s}}-\alpha_{\mathrm{f}}\right)^{2}\left(1-S_{1}\right)^{2} \mu_{2}}{K_{\mathrm{p}} k_{r 2}}
\end{aligned}
$$




$$
C_{3}=\frac{\alpha_{f}^{2} \mu_{2}}{K_{\mathrm{f}}}
$$

In equations (10.13)-(10.14), $K_{\mathrm{p}}$ is the intrinsic permeability of the nonfractured porous medium and $k_{\mathrm{ri}}$ is the relative permeability of phase $i, K_{\mathrm{f}}$ is the intrinsic permeability of the fractures and $\mu_{\mathrm{i}}$ is the viscosity of phase $i$. Equations (10.5)(10.11) form the final set of fifteen equations with fifteen unknowns $\bar{u}_{\mathrm{s}}, \bar{u}_{1}, \bar{u}_{2}$, $\bar{u}_{\mathrm{f}}, \bar{P}_{\mathrm{f}}, \bar{P}_{2}$ and $M$ (twelve displacements, two pressures and $M$ ).

\subsection{Compressional waves}

To investigate compressional waves, we apply divergence to equations (10.8)(10.11) to obtain

$$
\begin{aligned}
\left\langle\rho_{\mathrm{s}}\right\rangle \frac{\partial^{2} \epsilon_{\mathrm{s}}}{\partial t^{2}}= & a_{11}^{*} \nabla^{2} \epsilon_{\mathrm{s}}+a_{12} \nabla^{2} \epsilon_{1}+a_{13} \nabla^{2} \epsilon_{2}+a_{14} \nabla^{2} \epsilon_{\mathrm{f}}+\left(\frac{a_{14}}{\alpha_{\mathrm{f}}}-\frac{a_{13}}{\alpha_{2}}\right) \nabla^{2} M \\
& +C_{1}\left(\frac{\partial \epsilon_{1}}{\partial t}-\frac{\partial \epsilon_{\mathrm{s}}}{\partial t}\right)+C_{2}\left(\frac{\partial \epsilon_{2}}{\partial t}-\frac{\partial \epsilon_{\mathrm{s}}}{\partial t}\right)+C_{3}\left(\frac{\partial \epsilon_{\mathrm{f}}}{\partial t}-\frac{\partial \epsilon_{\mathrm{s}}}{\partial t}\right) \\
\left\langle\rho_{1}\right\rangle \frac{\partial^{2} \epsilon_{1}}{\partial t^{2}}= & a_{21} \nabla^{2} \epsilon_{\mathrm{s}}+a_{22} \nabla^{2} \epsilon_{1}+a_{23} \nabla^{2} \epsilon_{2}+a_{24} \nabla^{2} \epsilon_{\mathrm{f}} \\
& +\left(\frac{a_{24}}{\alpha_{\mathrm{f}}}-\frac{a_{23}}{\alpha_{2}}\right) \nabla^{2} M-C_{1}\left(\frac{\partial \epsilon_{1}}{\partial t}-\frac{\partial \epsilon_{\mathrm{s}}}{\partial t}\right) \\
\left\langle\rho_{2}\right\rangle \frac{\partial^{2} \epsilon_{2}}{\partial t^{2}=} & a_{31} \nabla^{2} \epsilon_{\mathrm{s}}+a_{32} \nabla^{2} \epsilon_{1}+a_{33} \nabla^{2} \epsilon_{2}+a_{34} \nabla^{2} \epsilon_{\mathrm{f}} \\
& +\left(\frac{a_{34}}{\alpha_{\mathrm{f}}}-\frac{a_{33}}{\alpha_{2}}\right) \nabla^{2} M-C_{2}\left(\frac{\partial \epsilon_{2}}{\partial t}-\frac{\partial \epsilon_{\mathrm{s}}}{\partial t}\right) \\
\left\langle\rho_{\mathrm{f}}\right\rangle \frac{\partial^{2} \epsilon_{\mathrm{f}}}{\partial t^{2}}= & a_{41} \nabla^{2} \epsilon_{\mathrm{s}}+a_{42} \nabla^{2} \epsilon_{1}+a_{43} \nabla^{2} \epsilon_{2}+a_{44} \nabla^{2} \epsilon_{\mathrm{f}} \\
& +\left(\frac{a_{44}}{\alpha_{\mathrm{f}}}-\frac{a_{43}}{\alpha_{2}}\right) \nabla^{2} M-C_{3}\left(\frac{\partial \epsilon_{\mathrm{f}}}{\partial t}-\frac{\partial \epsilon_{\mathrm{s}}}{\partial t}\right)
\end{aligned}
$$

where $\epsilon_{\mathrm{j}}=\nabla \cdot \bar{u}_{\mathrm{j}}$ and $a_{11}^{*}=a_{11}+4 G_{f r} / 3$. We state the dilatational plane harmonic waves propagating along the $\mathrm{z}$ direction by

$$
\begin{aligned}
& \epsilon_{\mathrm{j}}=B_{\mathrm{j}} e^{i(\xi z-\omega t)} \\
& M=B_{\mathrm{m}} e^{i(\xi z-\omega t)} \\
& P_{\mathrm{f}}=A_{\mathrm{j}} e^{i(\xi z-\omega t)} \\
& P_{\mathrm{p}}=A_{\mathrm{p}} e^{i(\xi z-\omega t)}
\end{aligned}
$$

where $B_{\mathrm{j}}, B_{\mathrm{m}}, A_{\mathrm{f}}$ and $A_{\mathrm{p}}$ are the wave amplitudes, $\xi$ is the wave number, $\omega$ is 
the frequency and $i$ is the imaginary number. The phase velocity is defined as $c=$ $\omega / \xi_{\mathrm{r}}$ where $\xi_{\mathrm{r}}$ is the real part of the wave number. The imaginary part of $\xi$ is called the attenuation coefficient. Substitution of equation (10.19) in equations (10.5)-(10.11) yields a set of homogeneous algebraic equations and for non-trivial solution the determinant of the coefficient matrix must be equal to zero. The reader is referred to Corapcioglu and Tuncay (1996b) for an expression of the coefficient matrix. For a given $\omega$, the determinant of the coefficient matrix equates to zero, and is known as the "dispersion equation" in wave mechanics literature. It is an eighth order polynomial in terms of wave number. The polynomial contains only the even powers of the wave number. Because the amplitude of the waves should decrease as they propagate, imaginary part of the wave number must be greater than zero. This implies the existence of four compressional waves. When $M=0$, i.e., no mass exchange between the porous blocks and fractures, the number of unknowns reduce to twelve $\left(\bar{u}_{\mathrm{s}}, \bar{u}_{1}, \bar{u}_{2}\right.$ and $\left.\bar{u}_{\mathrm{f}}\right)$ and the coefficient matrix reduces to a four by four matrix.

\subsection{Rotational waves}

To investigate the rotational waves, we apply curl operator to equations (10.8)(10.11)

$$
\begin{aligned}
& \left\langle\rho_{\mathrm{s}}\right\rangle \frac{\partial^{2} \Omega_{\mathrm{s}}}{\partial t^{2}}=G_{\mathrm{fr}} \nabla^{2} \Omega_{\mathrm{s}}+C_{1}\left(\frac{\partial \Omega_{1}}{\partial t}-\frac{\partial \Omega_{\mathrm{s}}}{\partial t}\right)+C_{2}\left(\frac{\partial \Omega_{2}}{\partial t}-\frac{\partial \Omega_{\mathrm{s}}}{\partial t}\right)+C_{3}\left(\frac{\partial \Omega_{\mathrm{f}}}{\partial t}-\frac{\partial \Omega_{\mathrm{s}}}{\partial t}\right) \\
& \left\langle\rho_{1}\right\rangle \frac{\partial^{2} \Omega_{1}}{\partial t^{2}}=-C_{1}\left(\frac{\partial \Omega_{1}}{\partial t}-\frac{\partial \Omega_{\mathrm{s}}}{\partial t}\right) \\
& \left\langle\rho_{2}\right\rangle \frac{\partial^{2} \Omega_{2}}{\partial t^{2}}=-C_{2}\left(\frac{\partial \Omega_{2}}{\partial t}-\frac{\partial \Omega_{\mathrm{s}}}{\partial t}\right) \\
& \left\langle\rho_{\mathrm{f}}\right\rangle \frac{\partial^{2} \Omega_{\mathrm{f}}}{\partial t^{2}}=-C_{3}\left(\frac{\partial \Omega_{\mathrm{f}}}{\partial t}-\frac{\partial \Omega_{\mathrm{s}}}{\partial t}\right)
\end{aligned}
$$

where $\Omega_{\mathrm{j}}=\nabla \times \bar{u}_{\mathrm{j}}$.

\subsection{Results}

Tuncay and Corapcioglu (1996b) solved the governing equations in terms of wave number for a given frequency. The phase velocity is defined as $c=\omega / \xi_{\mathrm{r}}$ where $\xi_{\mathrm{r}}$ is the real part of the wave number. The imaginary part of $\xi$ is called the attenuation coefficient. Van Genuchten's (1980) closed form expressions for the capillary pressure-saturation relations are employed to obtain $P_{\text {cap }}^{\prime}$ which appear in $a_{\mathrm{ij}}$ expressions. Van Genuchten proposed that 


$$
\frac{S_{2}-S_{\mathrm{r} 2}}{S_{\mathrm{m} 2}-S_{\mathrm{r} 2}}=\left(1+\left(\frac{\alpha P_{\mathrm{cap}}}{100}\right)^{n}\right)^{-m}
$$

where $P_{\text {cap }}$ is the capillary pressure $\left(\mathrm{N} / \mathrm{m}^{2}\right), \mathrm{S}_{2}$ is the water saturation, $S_{\mathrm{r} 2}$ is the irreducible water saturation, $S_{\mathrm{m} 2}$ is the upper limit of water saturation, $m=1-1 / n$, and $\alpha$ and $n$ are parameters.

From now on, we represent the compressional waves by " $P$ " and rotational wave by " $S$ ". Since there are four compressional waves, we will number them according to the magnitude of their phase velocity, P1 being the fastest. Tuncay and Corapcioglu showed the existence of four compressional and one rotational waves. The fastest wave (P1) is analogous to Biot's fast wave. The second wave (P2) arises because of the fluid phase in the fractures and it vanishes when the medium is not fractured. The third compressional wave (P3) corresponds to the slow wave of Biot's theory. The fourth compressional wave (P4) is due to the second fluid phase (non-wetting fluid) in the primary pores. The second, third and fourth compressional waves disappear when the frequency approaches zero and are associated with diffusive-type processes i.e., highly attenuated. The rotational wave $(S)$ is analogous to the rotational wave in elastic solids. All waves are dispersed and attenuated. Especially, the second, third and fourth compressional waves are highly attenuated. Because of the high attenuation, an experimental confirmation of these waves can be very difficult. Tuncay and Corapcioglu (1996b) numerically examined the frequency, saturation, volume fraction of fractures dependence of phase velocity and attenuation coefficient of body waves. The third and fourth compressional waves do not depend on the volume fraction of fractures. The phase velocities of the first compressional and rotational waves slightly change due to the volume fraction of fractures. However, Tuncay and Corapcioglu (1996b) observed a change in the order of magnitude of the attenuation coefficients of the first compressional and rotational waves due to the presence of the fractures. This can be explained by the high intrinsic permeability of the fractures.

\section{References}

Aifantis, E.C., 1979. On the response of fissured rocks. Proc. 16th Mid-western Mechanics Conf., Vol. 10, Kansas State Univ, Manhattan, Kansas, pp. 249-253.

Akbar, N., Dvorkin, J., and Nur, A., 1993. Relating P-wave attenuation to permeability. Geophysics, 58: 20-29.

Albert, D.G., 1993. A comparison between wave propagation in water-saturated and air-saturated porous materials. J. Appl. Phys., 73: 28-36.

Allen, N.F., Richart, F.E., and Woods, R.D., 1980. Fluid wave propagation in saturated and nearly saturated sands. J. Geotech. Eng., ASCE, 106: 235-254.

Anderson, T.B. and Jackson, R., 1967. A fluid mechnical description of fluidized beds. I\&EC Fundamentals, 6: 527-539.

Attenborough, K. and Chen, Y., 1990. Surface waves at an interface between air and air-filled poroelastic ground. J. Acoust. Soc. Am., 87: 1010-1016.

Auriault, J.L., 1980. Dynamic behaviour of a porous medium saturated by a Newtonian fluid. Int. J. Engng. Sci., 18: 775-785. 
Auriault, J.L., Borne, L., and Chambon, R., 1985. Dynamics of porous saturated media, checking of the generalized law of Darcy. J. Acoust. Soc. Am., 77: 1641-1650.

Auriault, J.L., Lebaique, O., and Bonnet, G., 1989. Dynamics of two immiscible fluids flowing through deformable porous media. Transport in Porous Media, 4: 105-128.

Baer, M.R., 1988. Numerical studies of dynamic compaction of inert and energetic granular material. J. Appl. Mech., 55: 36-43.

Baer, M.R. and Nunziato, J.W., 1986. A two-phase mixture theory for the deflagration to detonation transition (DDT) in reactive granular materials. Int. J. Multiphase Flow, 12: 861-889.

Barenblatt, G.I., Zheltow, I.P., and Kochina, T.N., 1960. Basic concepts in the theory of seepage of homogeneous liquids in fissured rocks. J. Appl. Math. Mech., 24: 1286-1303.

Barenblatt, G.I., 1963. On certain boundary value problems for the equations of seepage of a liquid in fissured rocks. J. Appl. Math. Mech., 27: 513-518.

Basak, P., and Madhav, M.R., 1978. Effect of the inertia term in one-dimensional fluid flow in deformable porous media. J. Hydrology, 38: 139-146.

Bazant, Z.P., and Krizek, R.J., 1975. Saturated sand as an inelastic two-phase medium. J. Eng. Mech., ASCE, 101: 317-332.

Bazant, Z.P., and Krizek, R.J., 1976. Endochronic constitutive law for liquefaction of sand. J. Eng. Mech. ASCE, 102: 225-238.

Bazant, Z.P., Ansal, A.M., and Krizek, R.J., 1982. Endochronic models for soils. In: G.N. Pande and O.C. Zienkiewicz (Editors), Soil Mechanics and Cyclic Loads. Wiley, Somerset, N.J., pp. 419428.

Bear, J. and Bachmat, Y., 1984. Transport phenomena in porous media-basic equation. In: J. Bear and M.Y. Corapcioglu (Editors), Fundamentals of Transport Phenomena in Porous Media. Martinus Nijhoff, Dordrecht, The Netherlands, pp. 3-61.

Bear, J. and Berkowitz, B., 1987. Groundwater flow and pollution in fractured rock aquifers. In: P. Novak (Editor), Developments in Hydraulic Engineering. Elsevier Applied Science, New York, N.Y., pp. 175-238.

Bear, J. and Corapcioglu, M.Y., 1981. Mathematical model for regional land subsidence due to pumping, I. Integrated aquifer subsidence equations based on vertical displacement only. Water Resour. Research, 17: 937-946.

Bear, J., Corapcioglu, M. Y., and Balakrishna, J., 1984. Modeling of centrifugal filtration in unsaturated deformable porous media. Adv. Water Resources, 7: 150-167.

Bear, J. and Corapcioglu, M.Y., 1989. Wave propagation in saturated porous media-Governing equations. In: D. Karamanlidis and R.B. Stout (Editors), Wave Propagation in Granular Media. ASME, New York, N.Y., 91-94.

Bedford, A. and Drumheller, D.S., 1979. A variational theory of porous media. Int. J. Solids Structures, 15: 967-980.

Bedford, A. and Stern, M., 1983. A model for wave propagation in gassy sediments. J. Acoust. Soc. Am., 73: 409-417.

Beebe, J.H., McDaniel, S.T., and Rubano, L.A., 1982. Shallow water transmission loss prediction using the Biot sediment model. J. Acous. Soc. Am., 71: 1417-1426.

Beranek, L.L., 1947. Acoustical properties of homogeneous, isotropic rigid tiles and flexible blankets. J. Acoust. Soc. Am., 19: 556-568.

Berryman, J.G., 1980a. Confirmation of Biot's theory. Appl. Phys. Lett., 37: 382-384.

Berryman, J.G., 1980b. Long wave length propagation of composite elastic media. J. Acoust. Soc. Am., 68: 1809-1831.

Berryman, J.G., 1981a. Elastic wave propagation in fluid-saturated porous media. J. Acoust. Soc. Am., 69: 416-424.

Berryman, J.G., 1981b. Elastic wave propagation in fluid-saturated porous media II. J. Acoust. Soc. Am., 69: 1754-1756.

Berryman, J.G., 1985. Scattering by a spherical inhomogeneity in fluid saturated porous medium. J. Math. Phys., 26: 1408-1419.

Berryman, J.G., 1986. Elastic wave attenuation in rocks containing fluids. Appl. Phys. Lett., 49: 552554. 
Berryman, J.G., 1986a. Effective medium approximation for elastic constants of porous solids with microscopic heterogeneity. J. Appl. Phys., 59: 1136-1140.

Berryman, J.G., 1986b. Elastic wave attenuation in rocks containing fluids. Appl. Phys. Lett., 49: 552-554.

Berryman, J.G., 1988. Seismic wave attenuation in fluid saturated porous media. Pageoph, 128: 423432.

Berryman, J.G. and Milton, G.W., 1985. Normalization constraint for variational bounds on fluid permeability. J. Chem. Phys., 83: 754-760.

Berryman, J.G. and Thigpen, L., 1985a. Effective constants for wave propagation through partially saturated porous media. Appl. Phys. Lett., 46: 722-724.

Berryman, J.G. and Thigpen, L., 1985b. Effective medium theory for partially saturated porous solids. In: Multiple Scattering of Waves in Random Media and Random Rough Surfaces. Penn. St. Univ., College Park, P.A., pp. 257-266.

Berryman, J.G. and Thigpen, L., 1985c. Linear dynamic poroelasticity with microstructure for partially saturated porous solids. J. Appl. Mech., 52: 345-350.

Berryman, J.G. and Thigpen, L., 1985d. Nonlinear and semilinear dynamic poroelasticity with microstructure. J. Mech. Phys. Solids, 33: 97-116.

Berryman, J.G., Thigpen, L., and Chin, R.C.Y., 1988. Bulk elastic wave propagation in partially saturated porous solids. J. Acoust. Soc. Am., 84: 360-373.

Beskos, D.E., 1989. Dynamics of saturated rocks, I: Equations of motion. J. Eng. Mech., ASCE, 115: 983-995.

Beskos, D.E. and Aifantis, E.C., (1986), 'On the theory of consolidation with double porosity. Int. J. Engng. Sci., 24: 1697-1716.

Beskos, D.E., Vgenopoulou, I., and Providakis, C.P., 1989a. Dynamics of Saturated rocks II: Body forces. J. Eng. Mech., ASCE, 115: 996-1016.

Beskos, D.E., Papadakis, C.N., and Woo, H.S., 1989b. Dynamics of saturated rocks, III: Rayleigh waves. J. Eng. Mech., ASCE, 115: 1017-1034.

Biot, M.A., 1941. General theory of three-dimensional consolidation. J. Appl. Physics, 12: 155-164.

Biot, M.A., 1956a. Theory of propagation of elastic wave in a fluid saturated porous solid, I. Low frequency range. J. Acoust. Soc. Am., 28: 168-178.

Biot, M.A., 1956b. Theory of propagation elastic waves in a fluid saturated porous solid, II. Higher frequency range. J. Acoust. Soc. Am., 28: 169-191.

Biot, M.A., 1962a. Mechanics of deformation and acoustic propagation in porous media. J. Appl. Phys., 33: 1482-1498.

Biot, M.A., 1962b. Generalized theory of acoustic propagation in porous dissipative media. J. Acoust. Soc. Am., 34: 1254-1264.

Biot, M.A. and Willis, D.G., 1957. The elastic coefficients of the theory of consolidation. J. Appl. Mech., 24: 594-601.

Bonnet, G., 1987. Basic singular solutions and boundary integral equations for a poroelastic medium in the dynamic range. J. Acoust. Soc., Am., 82: 1758-1762.

Bougacha, S., Tassoulas, J.L., and Roesset, J.M., 1993b. Analysis of foundations on fluid-filled poroelastic stratum. J. Eng. Mech., ASCE, 119: 1632-1648.

Bougacha, S., Roesset, J.M., and Tassoulas, J.L., 1993a. Dynamic stiffness of foundations on fluidfilled poroelastic stratum. J. Eng. Mech., ASCE, 119: 1649-1662.

Bougacha, S. and Tassoulas, J.L., 1991. Seismic analysis of gravity dams I: modelling of sediments. J. Eng. Mech., ASCE, 117: 1826-1837.

Boutin, C., Bonnet, G., and Bard, P.Y., 1987. Green functions and associated sources in infinite and stratified poroelastic media. Geophys. J. R. Astr. Soc., 90: 521-550.

Bowen, R.M., 1976. The theory of mixtures. In: A.C. Eringin (Editor), Continuum Physics, Vol. 3. Academic Press, New York, N.Y.

Bowen, R.M., 1980. Incompressible porous media models by use of the theory of mixtures. Int. J. Engng. Sci., 18: 1129-1148.

Bowen, R.M., 1982. Compressible porous media models by use of the theory of mixtures. Int. J. Engng. Sci., 20: 697-735. 
Bowen, R.M., and Lockett, R.R., 1983. Inertial effects in poroelasticity. J. Appl. Mech., 50: 334342.

Bowen, R.M. and Reinicke, K.M., 1978. Plane progressive waves in a binary mixture of linear elastic materials. J. Appl. Mech., 45: 493-499.

Brandt, H., 1955. A study of the speed of sound in porous granular media. J. Appl. Mech. 22: 479486.

Brandt, H., 1960. Factors affecting compressional wave velocity in unconsolidated marine sand sediments. J. Acoust. Soc. Am., 32: 171-179.

Briones, A.A. and Vehara, G., 1977. Soil elastic constants: I. Calculations from sound velocities. Soil Sci. Soc. Am. J., 41: 22-25.

Brutsaert, W., 1964. The propagation of elastic waves in unconsolidated unsaturated granular mediums. J. Geophys. Res., 69: 243-257.

Brutsaert, W. and Luthin, J.N., 1964. The velocity of sound in soils near the surface as a function of the moisture content. J. Geophys. Res., 69: 643-652.

Burridge, R. and Vargas, C.A., 1979. The fundamental solution in dynamic poroelasticity. Geophys. J.R. Am. Soc., 58: 61-90.

Burridge, R. and Keller, J.B., 1981. Poroelasticity equations derived from microstructure. J. Acoust. Soc. Am., 70: 1140-1146.

Butcher, B.M., Carroll, M.M., and Holt, A.C., 1974. Shock wave compaction of porous aluminum. J. Appl. Phys., 45: 3864-3875.

Carroll, M.M. and Holt, A.C., 1972. Static and dynamic pore collapse relations for ductile porous materials. J. Appl. Phys., 43: 1626-1635.

Chang, Y., Kabir, M.G., and Chang, Y., 1993. Micromechanics modeling for stress-strain behavior of granular soils I: Evaluation. J. Geotech. Eng., ASCE, 118: 1975-1992.

Chattopadhyay, A. and De, R.K., 1983. Love type waves in a porous layer with irregular interface. Int. J. Engng. Sci., 21: 1295-1303.

Chen, A.H.D., 1986. Effect of sediment on earthquake induced reservoir hydrodynamic response. J. Engng. Mech., ASCE, 112: 654-663.

Cheng, A.H.D., Badmus, T., and Beskos, D.E., 1991. Integral equation for dynamic poroelasticity in frequency domain with BEM solution. J. Eng. Mech., ASCE, 1136-1157.

Ching, C.S., Chang, Y., and Kabir, M.G., 1993. Micromechanics modeling for stress-strain behavior of granular soils I. Theory. J. Geotech. Eng., ASCE, 118: 1959-1974.

Cleary, M.P., 1977. Fundamental solutions for a fluid-saturated porous solid. Int. J. Solids Structures, 13: $785-806$.

Crochet, N.J. and Naghdi, P.M., 1966. On constitutive equations for flow of fluid through elastic porous media. Int. J. Engng. Sci., 4: 383-401.

Dagan. G., 1979. The generalization of Darcy's law for non-uniform flows. Water Resour. Res., 15: $1-17$.

Dalrymple, R.A. and Liu, P.L.F., 1978. Wave over soft muds: A two-layer fluid model. J Phys. Oceanog., 8: 1121-1131.

Dalrymple, R.A. and Liu, P.L.F., 1982. Gravity waves over a poroelastic seabed. ASCE Ocean Structural Dynamics Symposium, Proc., Oregon State University, Corvallis, O.R., pp. 181-195.

de Alba, P., Seed, H.B., and Chan, C.K., 1976. Sand liquefaction in large-scale simple shear tests. J. Geotech. Engng., ASCE, 102: 909-927.

de Boer, R., Ehlers, W., and Liu, Zhangfang, 1993. One-dimensional transient wave propagation in fluid-saturated incompressible porous media. Arc. Appl. Mech., 65: 59-72.

de Josselin de Jong, G., 1956. What happens in soil during pile driving? De Ingenieur, 68: B77-B88. de la Cruz, V. and Spanos, T.J.T., 1985. Seismic wave propagation in a porous medium. Geophysics, 50: 1556-1565.

de la Cruz, V. and Spanos, T.J.T., 1989. Thermomechanical coupling during seismic wave propagation in a porous medium. J. Geophys. Res., 94: 637-642.

Demars, K.R., 1983. Transient stresses induced in sandbed by wave loading. J. Geotech. Engng., ASCE, 109: 591-602.

Deresiewicz, H., 1960. The effect of boundaries on wave propagation in a liquid-filled porous solid: 
I. Reflection of plane waves at a free plane boundary (non-dissipative case). Bull. Seism. Soc. Am., 50: 599-607.

Deresiewicz, H., 1961. The effect of boundaries on wave propagation in a liquid-filled porous solid: II. Love waves in a porous layer. Bull. Seism. Soc. Am., 51: 51-59.

Deresiewicz, H. and Rice J.T., 1962. The effect of boundaries on wave propagation in a liquid-filled porous solid: III. Reflection of plane waves at free plane boundary (general case). Bull. Seism. Soc. Am., 52: 595-625

Deresiewicz, H., 1962. The effect of boundaries on wave propagation in a liquid-filled porous solid: IV. Surface waves in a half-space. Bull. Seism. Soc. Am., 52: 627-638.

Deresiewicz, H. and Rice J.T., 1964. The effect of boundaries on wave propagation in a liquid-filled porous solid: V. Transmission across a plane interface. Bull. Seism. Soc. Am., 54: 409-416.

Deresiewicz, H., 1964a. The effect of boundaries on wave propagation in a liquid-filled porous solid: VI. Love waves in a double surface layer. Bull. Seism. Soc. Am., 54: 417-423.

Deresiewicz, H., 1964b. The effect of boundaries on wave propagation in a liquid-filled porous solid: VII. Surface waves in a half-space in the presence of a liquid layer. Bull. Seism. Soc. Am., 54: $425-430$.

Deresiewicz, H. and Wolf, B., 1964. The effect of boundaries on wave propagation in a liquid-filled porous solid: VIII. Reflection of plane waves in an irregular boundary. Bull. Seism. Soc. Am., 54: $1537-1561$.

Deresiewicz, H., 1965. The effect of boundaries on wave propagation in a liquid-filled porous solid: IX. Love waves in a porous internal stratum. Bull. Seism. Soc. Am., 55: 919-923.

Deresiewicz, H., and Levy, A., 1967. The effect of boundaries on wave propagation in a liquid-filled porous solid: X. Transmission through a stratified medium. Bull. Seism. Soc. Am., 57: 381-391.

Derski, W., 1978. Equations of motion for a fluid saturated porous solid. Bull. Academia Pol. Sci., 26: $11-16$.

Digby, P.J. and Walton, K., 1989. Wave propagation through elastically-anisotropic fluid-saturated porous rocks. J. Appl. Mech., 56: 744-750.

Domenico, S.N., 1974. Effects of water saturation of sand reservoirs encased in shales. Geophysics, 29: 759-769.

Domenico, S.N., 1976. Effect of brine-gas mixture on velocity in an unconsolidated sand reservoir. Geophysics, 41: 882-894.

Drumheller, D.S., 1986. A theory for dynamic compaction of wet porous solids. Int. J. Solids Struct.

Duffy, J. and Mindlin, R.D., 1957. Stress-strain relations and vibration of a granular medium. J. Appl. Mech., 24: 585-593.

Duguid, J.O. and Lee, P.C.Y., 1977. Flow in fractured porous media. Water Resour. Res., 13: 558566.

Dunn, K.J., 1986. Acoustic attenuation in fluid-saturated porous cylinders at low frequencies. J. Acoust. Soc. Am., 79: 1709-1721.

Dutta, P.K., Farrell, D., Kalafut, J., 1990. A laboratory study of shock waves in frozen soil. In: D.S. Sodhi (Editor), Cold Regions Engineering. Proc. 6th Int. Specs. Conf., ASCE, New York, N.Y., pp. 54-70.

Elliott, S.E. and Wiley, B.F., 1975. Compressional velocities of partially saturated, unconsolidated sands. Geophysics, 40: $949-954$.

Fatt, I., 1959. The Biot-Willis elastic coefficients for a sandstone. J. Appl. Mech., 26: 296-297.

Feng, S. and Johnson, D.L., 1983. High-frequency acoustic properties of a fluid/porous solid interface I New surface mode. J. Acoust. Soc. Am., 74: 906-914.

Finjord, J., 1990. A solitary wave in a porous medium. Transport in Porous Media, 5: 591-607.

Finn, W.D.L., Byrne, P.M., and Martin, G.R., 1976. Seismic response and liquefaction of sands. J. Geotech. Engng., ASCE, 102: 841-856.

Finn, W.D.L., Lee, K.W., and Martin, G.R., 1977. An effective stress model for liquefaction. J. Geotech. Engng., ASCE, 103: 517-533.

Finn, W.D.L., Siddharthan, R., and Martin, G.R., 1983. Response of seafloor to ocean waves. J. Geotech. Eng., ASCE, 109: 556-572.

Foda, M.A. and Mei, C.C., 1983. A boundary layer theory for Rayleigh waves in a porous fluid-filled half space. Soil Dyn. Earth. Engng., 2: 62-65. 
Garg, S.K., 1971. Wave propagation effects in a fluid saturated porous solid. J. Geophys. Res., 76: $7947-7962$.

Garg, S.K., 1987. On balance laws for fluid saturated porous media. Mech. Materials, 6: 219-232.

Garg, S.K. and Kirsch, J.W., 1973. Steady shock waves in composite materials. J. Composite Materials, 7. $277-285$.

Garg, S.K., Nayfeh, A.H., and Good, A.J., 1974. Compressional waves in fluid-saturated elastic porous media. J. Appl. Phys., 45: 1968-1974.

Garg, S.K., Brownell, C.H., Pritchett, and Herrman, R.G., 1975. Shock wave propagation in fluid saturated porous media. J. Appl. Phys., 46: 702-713.

Garg, S.K. and Nayfeh, A.H., 1986. Compressional wave propagation in liquid and/or gas saturated elastic porous media. J. Appl. Phys., 60: 3045-3055.

Gassman, F., 1951. Elastic waves through a packing of spheres. Geophysics, 16: 673-685.

Geertsma, J., 1957. The effect of fluid pressure decline on volume changes of porous rocks. Trans. Am. Inst. Mining Metallurgical Eng., 210: 331-340.

Geertsma, J., 1974. Estimating the coefficient of inertial resistance in fluid flow through porous media. Soc. Petroleum Eng. J., 257: 445-450.

Geertsma, J. and Smit, D.C., 1961. Some aspects of elastic wave propagation in fluid saturated porous solids. Geophysics, 26: 160-180.

Ghaboussi, J. and Wilson, E.L., 1972. Variational formulation of dynamics of fluid saturated porous elastic solids. J. Engng. Mech., ASCE, 98: 947-963.

Ghaboussi, A.M. and Dikmen, S.U., 1978. Liquefaction analysis of horizontally layered sands. J. Geotech. Eng., ASCE, 104: 341-356.

Ghaboussi, J. and Dikmen, S.U., 1981. Liquefaction analysis for multidirectional shaking. J. Geotech. Engng., ASCE, 107: 605-627.

Ghaboussi, J. and Kim, K.J., 1984. Quasistatic and dynamic analysis of saturated and partially saturated soils. In: C.S. Desai and R.H. Gallagher (Editors), Mechanics of Engineering Materials. Wiley, Somerset, N.J., pp. 277-296.

Gokhale, S.S. and Krier, H., 1982. Modeling of unsteady two-phase reactive flow in porous beds of propellant. Prog. Energy Combust. Sci., 8: 1-39.

Goodman, M.A. and Cowin, S.C., 1972. A continuum theory for granular materials. Arch. Rat. Mech. Anal., 44: 249-266.

Grady, D.E., Moody, R.L., and Drumheller, D.S., 1986. Release equation of state of dry and water saturated porous calcite. Sandia Report SAND 86-2110. Sandia Nat. Lab., Albuquerque, N.M.

Green, A.E., and Naghdi, P.M., 1965. A dynamical theory of interacting continua. Int. J. Engng. Sci., 3: 231-241.

Gregory, A.R., 1976. Fluid saturation effects on dynamic elastic properties of sedimentary rocks. Geophysics, 41: 895-921.

Halpern, M. and Christiano P., 1986a. Response of poroelastic halfspace to steady-state harmonic surface tractions. Int. J. Num. Anal. Meth. Geomech., 10: 609-632.

Halpern, M. and Christiano P., 1986b. Steady-state harmonic response of a rigid plate bearing on a liquid-saturated poroelastic halfspace. Earth. Engrg. Struct. Dyn., 14: 439-454.

Hardin, B.O., 1965. The nature of damping in sands. J. Soil Mech. Found., ASCE, 91: 63-97.

Hardin, B.O. and Richart, F.E., 1963. Elastic wave velocities in granular soils. J. Soil Mech. Found., ASCE, 89: 33-65.

Hermann, W., 1968. Constitutive equation for the dynamic compaction of ductile porous materials. J. Appl. Phys., 40: 2490-2499.

Hassanzadeh, S., 1991. Acoustic modeling in fluid-saturated porous media. Geophysics, 56: 424-435.

Hermann, W., 1972. Constitutive equations for compaction of porous materials. In: Applied Mechanics Aspects of Nuclear Effects in Materials. Sandia Lab., Albuquerque, N.M.

Hiremath, M.S. and Sandhu, R.S., 1984. A computer program for dynamic response of layered saturated sand. Ohio St. Univ., Geotech. Eng. Rep. Columbus, O.H.

Hiremath, M.S., Sandhu, R.S., Morland, L.W., and Wolfe, W.E., 1988. Analysis of one-dimensional wave propagation in a fluid saturated finite soil column. Int. J. Num. and Analy. Meth. Geomech., 12: $121-139$. 
Holland, C.W. and Brunson, B.A., 1988. The Biot-Stoll sediment model: An experimental assessment. J. Acous. Soc. Am., 84: 1427-1443.

Hong, S.J., Sandhu, R.S., and Wolfe, W.E., 1988. On Garg's solution of Biot's equations for wave propagation in a one-dimensional fluid saturated elastic porous solid. Int. J. Num. Analy. Meth. Geomech., 12: 627-637.

Hovem, J.M., 1980. Viscous attenuation of sound in suspensions and high porosity marine sediment. J. Acoust. Soc. Am., 67: 1559-1573.

Hovem, J.M. and Ingram, G.D., 1979. Viscous attenuation of sound in saturated sand. J. Acoust. Soc. Am., 66: 1807-1812.

Hsieh, L. and Yew, C.H., 1973. Wave motions in a fluid-saturated porous medium. J. Appl. Mech., 40: $873-878$.

Iida, K., 1939. The velocity of elastic waves in sand. Bull. Earthquake Research Inst., Japan, 17: 738808.

Ishihara, K., 1967. Propagation of compressional waves in a saturated soil. In: Proc. Int. Symp. Wave Propagation and Dynamic Properties of Earth Materials. Univ. of New Mexico Press, Albuquerque, N.M., pp. 451-467.

Ishihara, K., 1970. Approximate forms of wave equations for water saturated porous materials and related dynamic modulus. J. Soc. Soil Mech. and Found. Eng., 10: 10-38.

Ishihara, K., Shimizu, K., and Yamada, Y., 1981. Pore water pressures measured in sand deposits during an earthquake. Soils and Foundations (Japan), 21: 85-100.

Ishiara, K. and Towhata, I., 1982. Dynamic response analysis of level ground based on the effective stress method. In: G.N. Pande and O.C. Zienkiewica (Editors), Soil Mechanics-Transient and Cyclic Loads. Wiley, Somerset, N.J., pp. 133-172.

Johnson, D.L., Plona, T., Plona, J., Scala, C., Pasierb, F., and Kojima, H., 1982. Tortuosity and acoustic slow waves. Phys. Rev. Lett., 49: 1840-1844.

Johnson, J.B., 1982. On the application of Biot's theory to acoustic wave propagation in snow. Cold Regions Sci. Tech., 6: 49-60.

Jones, J., 1969. Pulse propagation in a poroelastic solid. J. Appl. Mech., ASME, 36: 878-880.

Jones, J.P., 1961. Rayleigh waves in a porous, elastic, saturated solid. J. Acoust. Soc. Am., 33: 959962.

Jones, T. and Nur, A., 1983. Velocity and attenuation in sandstone at elevated temperatures and pressures. Geophys. Res. Lett., 10: 140-143.

Kansa, E.J., 1987. A guide to the transient three phase porous flow model implemented in the twodimensional Cray-tensor code: Physics, numerics, and code description. Lawrence Livermore Nat. Lab. Rep. UCID-21260.

Kansa, E.J., 1989. The response of shocks in unsaturated geological media under a wide range of permeabilities. In: D. Karamanlidis and R.B. Stout (Editors), Wave Propagation in Granular Materials. ASME, New York, N.Y., pp. 95-101.

Kansa, E.J., 1988. Numerical solution of three phase porous flow under shock conditions. Mathl. Comput. Modelling, 11: 180-185.

Kansa, E.J., Kirk, T.M., and Swift, R.P., 1987. Multiphase flow in geological materials: Dynamic loading theory and numerical modeling. In: AIChE Symposium Series 257, Vol. 83, pp. 206-210.

Katsube, N., 1985. The constitutive theory for fluid-filled porous materials. J. Appl. Mech., 52: 185189.

Katsube, N., and Carroll, M.M., 1987. The modified mixture theory for fluid-filled porous materials., J. Appl. Mech., 54: 35-40.

Khaled, M.Y., Beskos, D.E., and Aifantis, E.C., 1984) On the porosity of consolidation with double porosity - III: A finite element formulation. Int. J. Num. Meth. Eng., 8: 101-123.

Kim, Y.K. and Kingsbury, H.G., 1979. Dynamic characterization of poroelastic materials. Exp. Mech., 252-258.

Klimentos, T. and McCann, C., 1990. Relationships among compressional wave attenuation, porosity, clay content, and permeability in sandstones. Geophysics, 55: 998-1014.

Korringa, J., 1981. On the Biot-Gassman equations for the elastic moduli of porous rocks. (Critical comment on a paper by J.G. Berryman.) J. Acoust. Soc. Am., 70: 1752-1753.

Lebaigue, O.D., Bonnet, G.I., and Auriault, J.D., 1987. Transparency ultrasonic tests on a thin plate 
of unsaturated porous medium application to wet paper. Ultrasonics Int. 87 Conf. Proc. London, pp. 635-640.

Levy, T., 1979. Propagation of waves in a fluid-saturated porous elastic solid. Int. J. Engng. Sci., 17: 1005-1014.

Levy, T. and Sanchez-Palencia, E., 1977. Equations and interface conditions for acoustic phenomena in porous media. J. Math. Analy. Applications, 61: 813-834.

Liou, C.P., Streeter, V.L., and Richart, F.E., 1977. Numerical model for liquefaction. J. Geotech. Engng., ASCE, 103: 589-606.

Liu, P.L.F. and Darlrymple, R.A., 1984. The damping of gravity water-waves due to percolation. Coastal Eng., 8: 33-49.

Liu, Q.R. and Katsube, N., 1990. The discovery of a second kind of rotational wave in a fluid-filled porous material. J. Acoust. Soc. Am., 88: 1045-1053.

Loret, B., 1990. Acceleration waves in elastic-plastic porous media: Interlacing and seperation properties. Int. J. Engng. Sci., 28: 1315-1320.

Loret, B. and Pervost, J.H., 1991. Dynamic strain localization in fluid-saturated porous media. J. Eng. Mech., ASCE, 117: 907-922.

Lovera, O.M., 1987. Boundary conditions for a fluid-saturated porous solid. Geophysics, 174-178.

Madsen, O.S., 1978. Wave-induced pore-pressures and effective stresses in a porous bed. Geotechnique, 28: 377-393.

Mann, R.W., 1979. Elastic Wave Propagation in Paper. Ph.D. Dissertation. Lawrence Univ., Appleton, W.I.

Martin, G.R., Finn, W.D.L., and Seed, H.B., 1975. Fundamentals of liquefaction under cyclic loading. J. Geotech. Engng., ASCE, 101: 423-438.

Mansouri, T.A., Nelson, J.D., and Thompson, E.G., 1983. Dynamic response and liquefaction of earth dam. J. Geotech. Eng., ASCE, 109: 89-100.

Massel, S.R., 1976. Gravity waves propagated over permeable beds. J. Waterways, Harbours, and Coastal Eng., ASCE, 102: 11-21.

Mavko, G.M. and Nur, A., 1979. Wave attenuation in partially saturated rocks. Geophysics, 44, 161178.

McCann, C. and McCann, D.M., 1969. The attenuation of compressional waves in marine sediments. Geophysics, 34: 882-892.

Mei, C.C. and Foda, M.A., 1981. Wave-induced responses in a fluid-filled poro-elastic solid with a free surface-A boundary layer theory. Geophys. J.R. Astr., Soc., 66: 597-631.

Mei, C.C. and Foda, M.A., 1982. Boundary layer theory of waves in a poro-elastic sea bed. In: G.N. Pande and O.C. Zienkiewicz (Editors), Soil Mechanics-Transient and Cyclic Loads. Wiley, Somserset, N.J., pp. 17-35.

Mei, C.C. and Mynett, A.E., 1983. Two-dimensional stresses in a saturated poro-elastic foundation beneath a rigid structure, I. A dam in river. Int. J. Numer. Analy. Meth. Geomech., 7: 57-74.

Mei, C.C., Boon I.S., and Chen, Y.S., 1985. Dynamic response in a poro-elastic ground induced by a moving air pressure. Wave Motion, $7:$ 129-141.

Misra, H.C., 1965. Permeability of Porous Media to Transient Flow'. Ph.D. thesis, Univ. of Wisconsin, Madison, W.I.

Misra, H.C. and Monkmeyer, P.L., 1966. On the response of sound waves to the permeability of a porous medium. Presented at the 15th An. ASCE Hyd. Div. Conf., Madison, W.I.

Mochizuki, S., 1982. Attenuation in partially saturated rocks. J. Geophysical Res., 87: 8598-8604.

Morland, L.W., 1972. A simple constitutive theory for a fluid-saturated porous solid. J. Geophys. Res., 77: 890-900.

Morland, L.W., Sandhu, R.S., Wolfe, W.C., and Hiremath, M.S., 1987. Wave propagation in a fluidsaturated elastic layer. Geotechnical Eng., Rep. No. 25, Ohio State Univ., Columbus, O.H.

Morland, L.W., Sandhu, R.S., and Wolfe, W.E., 1988. Uni-axial wave propagation through fluidsaturated elastic soil layer. In: G. Swoboda (Editor), Numerical Methods in Geomechanics. Innsbruck, 1988, Balkema, Rotterdam, pp. 213-220.

Morse, R.W., 1952. Acoustic propagation in granular media. J. Acoust. Soc. Am., 24: 696-700.

Moshagen, H. and Torum, A., 1975. Wave induced pressures in permeable seabeds. J. Waterways, Harbours and Coastal Eng., ASCE, 101: 49-57. 
Murphy, W.F., 1982. Effects of partial water saturation on attenuation in Massilon sandstone and Vycor porous glass. J. Acoust. Soc., Am., 71: 1458-1468.

Murphy, W.F., 1984. Acoustic measures of partial gas saturation in tight sandstones. J. Geophysical Res., 89: 11549-11559.

Murphy, W.F., Winkler, K.W., and Kleinberg, R.L., 1986. Acoustic relaxation in sedimentary rocks: Dependence on grain contacts and fluid saturation. Geophysics, 51: 757-766.

Mynett, A.E. and Mei, C.C., 1982. Wave-induced stresses in a saturated poro-elastic sea bed beneath a rectangular caisson. Geotechnique, 32: 235-247.

Mynett, A.E. and Mei, C.C., 1983. Earthquake induced stresses in a poro-elastic foundation supporting a rigid structure. Geotechnique, 33: 293-303.

Nagy, P.B., 1993. Slow wave propagation in air-filled permeable solids. J. Acoust. Soc. Am., 93: 3224-3234.

Nataraja, M.S. and Gill, H.S., 1983. Ocean wave-induced liquefaction analysis. J. Geotech. Eng., ASCE, 109: 573-590.

Nikolaevskij, V.N., 1990. Mechanics of Porous and Fractured Media, World Scientific, Singapore.

Nolle, A.W., Hoyer, W.A., Mifsud, J.F., Runyan, W.R., and Ward, M.B., 1963. Acoustical properties of water-filled sands. J. Acoust. Soc. Am., 35: 1394-1408.

Norris, A.N., 1985. Radiation from a point source and scattering theory in a fluid-saturated porous solid. J. Acoust. Soc. Am., 77: 2012-2023.

Norris, A.N., 1993. Low-frequency dispersion and attenuation in partially saturated rocks. J. Acoust. Soc. Am., 94: 359-370.

Nunziato, J.W. and Walsh, E.K., 1977. On the influence of void compaction and material nonuniformity on the propagation of one-dimensional acceleration waves in granular materials. Arch. Rational Mech. Anal, 64: 299-316.

Nunziato, J.W., Kennedy, J.E., and Walsh, E., 1978. The behaviour of one-dimensional acceleration waves in an inhomogeneous granular solid. Int. J. Engng. Sci., 16: 637-648.

Nur, A. and Booker, J.R., 1972. Aftershocks caused by pore fluid flow? Science, 885-887.

Ogushwitz, P.R., 1985. Applicability of the Biot theory: I. Low porosity materials II. Suspensions, III. Wave speeds versus depth in marine sediments. J. Acoust. Soc. Am., 77: 429-464.

Paria, G., 1963. Flow of fluids through porous deformable solids. Appl. Mech. Rev., 16.

Parra, J.O., 1991. Analysis of elastic wave propagation in stratified fluid-filled porous media for interwell seismic applications. J. Acoust. Soc. Am., 90: 2557-2575.

Pascal, H., 1986. Pressure wave propagation in a fluid flowing through a porous medium and problems related to interpretation of Stoneley's wave attenuation in acoustical well logging. Int. J. Engng. Sci., 24: 1553-1570.

Pecker, C. and Deresiewicz, H., 1973. Thermal effects on wave propagation in liquid filled porous media. Acta Mechanica, 16: 45-64.

Philippacopoulos, A.J., 1987. Waves in a partially saturated layered half-space analytic formulation. Bull. Seismological Soc. Amer., 77: 1838-1853.

Plona, T.J., 1980. Observation of a second bulk compressional wave in a porous medium at ultrasonic frequencies. App. Phys. Lett., 36: 259-261.

Plona, T.J. and Johnson, D.L., 1984. Acoustic properties of porous systems: I. Phenomenological description. In: D.L. Johnson and P.N. Sen (Editors), Physics and Chemistry of Porous Media, Vol. 107. American Institute of Physics, New York, N.Y., pp. 89-104.

Powers, J.M., Stewert, D.S., and Krier, H., 1989. Analysis of steady compaction waves in porous materials. J. Appl. Mech., 56: 15-24.

Prassad, M. and Meissner, R., 1992. Attenuations mechanisms in sands: Laboratory versus theoretical Biot data. Geophysics, 57: 710-719.

Predeleanu, M., 1984. Development of boundary element method to dynamic problems for porous media. Appl. Math. Modelling, 8: 378-382.

Prevost, J.H., 1980. Mechanics of continuous porous media. Int. J. Engng. Sci., 18: 787-800.

Prevost, J.H., 1982. Nonlinear transient phenomena in saturated porous media. Computer Meth. in Appl. Mech. Engng., 20: 3-18.

Prevost, J.H., 1984. Non-linear transient phenomena in soil media. In: C.S. Desai and R.H. Gallagher (Editors). Mechanics of Engineering Materials. Wiley, Somerset, N.J., pp. 515-533. 
Prevost, J.H., 1985. Wave propagation in fluid-saturated porous media: An efficient finite element procedure. Soil Dynamics Earthquake Eng., 4: 183-202.

Pride, S.R., Gangi, A.F., and Morgan, F.D., 1992. Deriving equations of motion for porous isotropic media. J. Acoust. Soc. Am., 92: 3278-3290.

Putnam, J.A., 1949. Loss of wave energy due to percolation in a permeable sea bottom. Trans. Am. Geophys. Union, 30: 349-366.

Raats, P.A.C., 1969. The effect of a finite response time upon the propagation of sinusoidal oscillations of fluids in porous media. ZAMP, 20: 936-946.

Raats, P.A.C., 1972. The role of inertia in the hydrodynamics of porous media. Arch. Rat. Mech. Analysis, 44: 267-280.

Raats, P.A.C. and Klute, A., 1969. Transport in soils: The balance of momentum. Soil Sci. Soc. Amer. Proc., 32: 452-456.

Rahman, M.S., Seed, H.B., and Booker, J.R., 1977. Pore pressure development under offshore gravity structures. J. Geotech. Engng., ASCE, 103: 1419-1436.

Reid, R.O. and Kajivra, K., 1957. On the damping of gravity waves over a permeable seabed. Trans. Am. Geophys. Union, 38: 662-666.

Rice, J.R. and Cleary, M.P., 1976. Some basic stress-diffusion solutions for fluid saturated elastic porous media with compressible constituents. Rev. Geophys. Space Phys., 14: 227-241.

Richart, F.E., Jr., Hall, J.R., Jr., and Woods, R.D., 1970. Vibrations of Soils and Foundations. Prentice Hall, Englewood Cliffs, N.J.

Ross, C.A., Thompson, P.Y., Charlie, W.A., and Dohering, D.O., 1989. Transmission of pressure waves in partially saturated soils. Experimental Mech., March, 80-83.

Sadd, M.H., Shukla, A., Mei, H., and Zhu, C.Y., 1989. The effect of voids and inclusion on wave propagation in granular materials. In: G.J. Weng, M. Taya, and H. Abe (Editors), Micromechanics and Homogeneity. Springer-Verlag, New York, N.Y.

Sadd, M.H. and Hossain, M., 1989. Wave propagation in distributed bodies with applications to dynamic soil behaviour. J. Wave-Material Interaction, 4.

Salin, D. and Schon, W., 1981. Acoustics of water saturated packed glass spheres. J. Phys. Lett., 42: 477-480.

Sanchez-Palencia, E., 1980. Non-Homogeneous Media and Vibration Theory. Springer-Verlag, New York, N.Y.

Sandhu, R.S. and Pister, K.S., 1970. A variational principle for linear, coupled field problems in continuum mechanics. Int. J. Eng. Sci., 8: 989-997.

Sandhu, R.S., Wolfe, E., and Shaw, H.C., 1989. Dynamic response of saturated soils using three-field formulation. Soil Dynamics Earthquake Eng., 8:

Sandhu, R.S. and Hong, S.J.(1987. Dynamics of fluid saturated soils-variational formulation. Int. J. Num. Analy. Meth. Geomech., 11: 241-255.

Santos, J.E., 1986. Elastic wave propagation in fluid-saturated porous media, I: The existence and uniqueness theorems. Math. Model. Num. Analy., 20: 113-128.

Santos, J.E., Orena, E.J., 1986. Elastic wave propagation in fluid-saturated porous media, II: The Galerkin procedures. Math. Model. Num. Analy., 20: 129-139.

Santos, J.E., Corbero, J.M., and Douglas, J., 1990a. Static and dynamic behaviour of a porous solid. J. Acoust. Soc. Am., 87: 1428-1438.

Santos, J.E., Douglas, J., Corbero, J.M., and Lovera, O.M., 1990b. A model for wave propagation in a porous medium saturated by a two-phase fluid. J. Acoust. Soc. Am., 87: 1439-1448.

Santos, J.E., Corbero, J.M., Ravazzoli, C.L., and Hensley, J.L., 1992. Reflection and transmission coefficients in fluid-saturated porous media. J. Acoust. Soc. Am., 91: 1911-1923.

Sawicki, A. and Morland, L.W., 1985. Pore pressure generation in a saturated sand layer subjected to a cyclic horizontal acceleration at its base. J. Mech. Phys. Solids, 33: 545-559.

Schmidt, E.J., 1988. Wideband acoustic response of fluid-saturated porous rocks: Theory and preliminary results using wave guided samples. J. Acoust. Soc. Am., 83: 2027-2024.

Schuurman, I.E., 1966. The compressibility of an air/water mixture and a theoretical relation between the air and water pressures. Geotechnique, 16: 269-281.

Schwartz, L.M., 1984. Acoustic properties of porous systems: Microscopic description. In: D.L. 
Johnson and P.N. Sen (Editors), Physics and Chemistry of Porous Media. Am. Inst. Phys., Vol. 107, pp. $105-118$.

Scott, P.H. and Rose, W., 1953. An explanation of the Yuster effect. J. Petr. Technol, , 5: 19-20.

Scott, R.F., 1986. Solidification and consolidation of a liquefied sand column. Soils and Foundations (Japan), 26: 23-31.

Seed, H.B., Martin, P.P., and Lysmer, H., 1976. Pore water pressure changes during soil liquefaction.

J. Geotech. Engng., ASCE, 102: 323-346.

Seed, H.B. and Rahman, M.S., 1978. Wave induced pore pressure in relation to ocean floor stability of cohesionless soils. Marine Geotechnology, 3 .

Seed, H.B. and Idriss, I.M., 1982. On the importance of dissipation effects in evaluating pore pressure changes due to cyclic loading. In: G.N. Pande and O.C. Zienkiewicz (Editors), Soil MechanicsTransient and Cyclic Loads. Wiley, Somerset, N.J., pp. 53-70.

Sharma, M.D. and Gogna, M.L., 1991a. Propagation of Love waves in an initially stressed medium consisting of a slow elastic layer lying over a liquid-saturated porous half-space. J. Acoust. Soc. Am., 89: 2584-2588.

Sharma, M.D. and Gogna, M.L., 1991b. Wave propagation in anisotropic liquid-saturated porous solids. J. Acoust. Soc. Am., 90: 1068-1073.

Shukla, A. and Zhu, Y., 1988. Influence of the microstructure of granular media on wave propagation and dynamic load transfer. J. Wave-Material Interaction, 3: 249-265.

Siddharthan, R., 1987. Wave-induced displacements in seafloor sand. Int. J. Num. Analy. Meth. Geomech., 11: 155-170.

Slattery, J.C., 1967. Flow of viscoelastic fluids through porous media. AIChE J., 14: 1066-1071.

Sleath, J.F.A., 1970. Wave induced pressures in beds of sand. J. Hydraul. Div., ASCE, 96: 367-378.

Smith, D.T., 1974. Acoustic and mechanical loading of marine sediments. In: L. Hampton (Editor), Physics of Sound in Marine Sediments. Plenum, New York, N.Y., pp. 41-61.

Smith, P.G. and Greenkorn, R.A., 1972. Theory of acoustical wave propagation in porous media. J. Acoust. Soc. Am., 52: 247-253.

Smith, P.G., Greenkorn, R.A., and Barile, R.G., 1974a. Infrasonic response characteristics of gas and liquid porous media. J. Acoust. Soc. Am., 56: 781-788.

Smith, P.G., Greenkorn, R.A., and Barile, R.G., 1974b. Theory of transient pressure response of fluid filled porous media. J. Acoust. Soc. Am., 56: 789-795.

Spooner, J.A., 1971. Unsteady Inertial Effects in Fluid Flow Through Porous Media. Ph.D. Thesis, Univ. of Wisconsin, Madison, W.I.

Stoll, R.D., 1974. Acoustic waves in saturated sediments. In: L. Hampton (Editor), Physics of Sound in Marine Sediments. Plenum, New York, N.Y., pp. 19-39.

Stoll, R.D., 1977. Acoustic waves in ocean sediments. Geophysics, 42: 715-725.

Stoll, R.D., 1979. Experimental studies of attenuation in sediments. J. Acoust. Soc. Am., 66: 11521160.

Stoll, R.D., 1980. Theoretical aspects of sound transmission in sediments. J. Acoust. Soc. Am., 68: 1341-1350.

Stoll, R.D. and Bryan, G.M., 1970. Wave attenuation in saturated sediments. J. Acoust. Soc. Am., 47: 1440-1447.

Stoll, R.D. and Kan, T.K., 1981. Reflection of acoustic waves at a water-sediment interface. J. Acoust. Soc. Am., 70: 149-156.

Streeter, V.L., Wylie, E.B., and Richart, F.E., 1974. Soil motion computations by characteristics method. J. Geotech. Engng., ASCE, 100: 247-263.

Sun, F., Banks, P., and Peng, H., 1993. Wave propagation theory in anisotropic periodically layered fluid-saturated porous media., J. Acoust. Soc. Am., 93: 1277-1285.

Tajuddin, M., 1984. Rayleigh waves in a poroelastic half-space. J. Acoust. Soc. Am., 75: 682-684.

Tajuddin, M. and Moiz, A.A., 1984. Rayleigh waves on a convex cylindrical poroelastic surface Part II. J. Acoust. Soc. Am., 76: 1252-1254.

Tajuddin, M. and Ahmed, S.I., 1991. Dynamic interaction of a poroelastic layer and a half space. J. Acoust. Soc. Am., 89: 1169-1175.

Tiller, F.M., 1975. Compressible cake filtration. In: K.J. Ives (Editors), The Scientific Basis of Filtration. NATO/ASI Series No. 2, Noordhoff-Leyden, The Netherlands, pp. 315-397. 
Tuncay, K. and Corapcioglu, M.Y., 1996a. Wave propagation in fractured porous media. Transport in Porous Media, 23: 237-258.

Tuncay, K. and Corapcioglu, M.Y., 1996b. Body waves in fractured porous media saturated by two immiscible newtonian fluids. Transport in Porous Media, 23: 259-273.

Turgut, A. and Yamamoto, T., 1990. Measurements of acoustic wave velocities and attenuation in marine sediments. J. Acoust. Soc. Am., 87: 2376-2383.

Valanis, K.C., 1971. A theory of viscoplasticity without a yield surface. Arch. of Mech., 23: 517-555.

Valanis, K.C., and Read, H.E., 1982. A New endochronic plasticity model for soils. In: G.N. Pande and O.C. Zienkiewicz (Editors), Soil Mechanics and Cyclic Loads. Wiley, Somerset, N.J., pp. 375417.

van der Grinten, J.G.M., Van Dongen, M.E.H., and Van der Kogel, H., 1985. A shock-tube technique for studying pore pressure propagation in a dry and water saturated porous medium. J. Appl. Phys., 58: 2937-2942.

van der Grinten, J.G.M., Smits, M.A., Van der Kogel, H., and Van Dongen, M.E.H., 1987a. Shock induced wave propagation in and reflection from a porous column partially saturated with water. In: H. Gronig (Etitor), Proc. 6th Int. Symp. Shock Tubes and Waves. VCH, pp. 357-362.

van der Grinten, J.G.M., van Dorgen, M.E.H., and van der Kogel, H., 1987b. Strain and pore pressure propagation in a water-saturated porous medium. J. App. Phys., 62: 4682-4687.

van Genuchten, M. Th., 1980. A closed form equation for predicting the hydraulic coductivity of unsaturated soils. Soil Sci. Soc. Am. J., 44: 892-898.

Vardoulakis, I., 1987. Compression induced liquefaction of water saturated granular media. In: C.S Desai (Editor), Constitutive Laws for Engineering Materials. Elsevier, New York, N.Y., pp. 647656.

Vardoulakis, I. and Beskos, D.E., 1986. Dynamic behavior of nerly saturated porous media. Mech. Matls., 5: 87-108.

Verruijt, A., 1969. Elastic storage of aquifers. In: R.J.M. DeWeist (Editor), Flow Through Porous Media. Academic Press, New York, N.Y., pp. 331-376.

Verruijt, A., 1982. Approximations to cyclic pore pressures caused by sea waves in a poroelastic halfplane. In: G.N. Pande and Zienkiewicz (Editors), Soil Mechanics-Transient and Cyclic Loads. Wiley, Somerset, N.J., pp. 37-51.

Verruijt, A., 1984. The theory of consolidation. In: J. Bear and M.Y. Corapcioglu (Editors), Fundamentals of Transport Phenomena in Porous Media. Martinus Nijhoff, Dordrecht, The Netherlands, pp. 349-368.

Weng, X. and Yew, C.H., 1990. The leaky Rayleigh wave and Scholte wave at an interface between water and porous sea ice. J. Acoust. Soc. Am., 87: 2481-2488.

Whitaker, S., 1967. Diffusion and dispersion in porous media. AIChE J., 13: 420-427.

White, J.E., 1975. Computed seismic speeds and attenuation in rocks with partial gas saturation. Geophysics, 40: 224-232.

Wiggert, D.C. and Wylie, E.B., 1976. Numerical predictions of two-dimensional transient groundwater flow by the method of characteristics. Water Resour. Res., 12: 971-977.

Wijesinghe, A.M. and Kingsbury, H.B., 1979. On the dynamic behaviour of poroelastic materials. J. Acoust. Soc. Am., 65: 90-95.

Wijesinghe, A.M. and Kingsbury, H.B., 1980. Response to dynamic surface pressure distributions. J. Geotech. Engng., ASCE, 106: 1-15.

Wilson, R.K. and Aifantis, E.C., 1982. On the theory of consolidation with double porosity. Int. J. Eng. Sci., 22: 1009-1035.

Wilson, R.K. and Aifantis, E.C., 1984. A double porosity model for acoustic wave propagation in fractured-porous rock. Int. J. Eng. Sci., 22: 1209-1217.

Wu, K., Xue, Q., and Adler, L., 1990. Reflection and transmission of elastic waves from a fluidsaturated porous solid boundary. J. Acoust. Soc. Am., 87: 2349-2358.

Wylie, E.B., 1976. Transient aquifer flows by characteristics method. J. Hyd. Div., ASCE, 102: 293305.

Wyllie, M.R.J., Gardner, G.H.F., and Gregory, A.R., 1962. Studies of elastic wave attenuation in porous media. Geophysics, 27: 569 . 
Yamamoto, T., Koning, H.L., Sellmeijer, H., and Van Hijum, E., 1978. On the response of a poroelastic bed to water waves. J. Fluid Mech., 87(1): 192-206.

Yamamoto, T. and Schuckman, B., 1984. Experiments and theory of wave-soil interactions. J. Eng. Mech., ASCE, 110: 95-112.

Yamamoto, T. and Takahashi, S., 1983. Physical modeling of sea-seabed interactions. J. Eng. Mech., ASCE, 109: 54-72.

Yew, C.H. and Jogi, P.N., 1976. Study of wave motions in fluid-saturated porous rocks. J. Acoust. Soc. Am., 60: 2-8.

Yin, C.S., Batzle, M.I., and Smith, B.J., 1992. Effects of partial liquid/gas saturation on extensional wave attenuation in berea sandstone. Geophys. Res. Lett., 19: 1399-1402.

Yuster, S.T., 1951. Theoretical considerations of multiphase flow in idealized capillary system. Proc. Third World Petr. Cong., The Hauge, 2: 436-445.

Zhu, X. and McMechan, G.A., 1991. Numerical simulation of seismic responses of poroelastic reservoirs using Biot theory. Geophysics, 56: 328-339.

Zienkiewicz, O.C., 1982. Basic formulation of static and dynamic behaviour of soil and other porous media: In: J.B. Martins (Editors), Numerical Methods in Geomechanics. Reidel, Dordrecht, The Netherlands, pp. $39-55$.

Zienkiewicz, O.C., Chang, C.T., and Hinton, E., 1978. Nonlinear seismic response and liquefaction. Int. J. Num. Analy. Meth. Geomech., 2: 381-404.

Zienkiewicz, O.C, Chang, C.T., and Battess, P., 1980. Drained, undrained, consolidating, and dynamic behaviour assumptions in soils, Limits of validity. Geotechnique, 30: 385-395.

Zienkiewicz, O.C., Leung, K.H., Hinton, E., and Chang, C.T., 1982a. Liquefaction and permanent deformation under dynamic conditions. Numerical solution and constitutive relations. In G.N. Pande and O.C. Zienkieqicz (Editors), Soil Mechanics and Cyclic Loads, Wiley, Somerset, N.J., pp. $71-103$.

Zienkiewicz, O.C., Leung, K.H., and Hinton, E., 1982b. Earthquake response behaviour of soils with drainage. Univ. College of Swansea, Inst. for Num. Meth. in Engng. Rep. C/R/404/82.

Zienkiewicz, O.C. and Bettess, P., 1982c. Soils and other saturated media under transient, dynamic conditions: General formulation and the validity of various simplifying assumptions. In: G.N. Pande and O.C. Zienkiewicz (Editors), Soil Mechanics - Transient and Cyclic Loads. Wiley, Somerset, N.J., pp. 1-16.

Zienkiewicz, O.C. and Shiomi, T. 1984. Dynamic behaviour of saturated porous media, the generalized Biot formulation and its numerical solution. Int. J. Num. Analy. Meth. Geomech., 8: 71-96.

Zolotarjew, P.P. and Nikolaevskij, V.N., 1965. Propagation of stress and pore pressure discontinuities in water saturated soil. Izvestija Akademii Nauk. Mechanika, No. 1 (in Russian), 191-196.

Zwikker, C. and Kosten, C.W., 1949 Sound absorbing materials. Elsevier, New York, N.Y. 